\title{
Long-range deformations for integrable spin chains
}

\author{
Till Bargheer, Niklas Beisert and Florian Loebbert \\ Max-Planck-Institut für Gravitationsphysik, Albert-Einstein-Institut, Am Mühlenberg 1, \\ 14476 Potsdam, Germany \\ E-mail: bargheer@aei.mpg.de, nbeisert@aei.mpg.de and loebbert@aei.mpg.de
}

Received 23 February 2009, in final form 29 May 2009

Published 25 June 2009

Online at stacks.iop.org/JPhysA/42/285205

\begin{abstract}
We present a recursion relation for the explicit construction of integrable spin chain Hamiltonians with long-range interactions. Based on arbitrary shortrange (e.g. nearest neighbor) integrable spin chains, it allows us to construct an infinite set of conserved long-range charges. We explain the moduli space of deformation parameters by different classes of generating operators. The rapidity map and dressing phase in the long-range Bethe equations are a result of these deformations. The closed chain asymptotic Bethe equations for long-range spin chains transforming under a generic symmetry algebra are derived. Notably, our construction applies to generalizations of standard nearest neighbor chains such as alternating spin chains. We also discuss relevant properties for its application to planar $D=4, \mathcal{N}=4$ and $D=3, \mathcal{N}=6$ supersymmetric gauge theories. Finally, we present a map between long-range and inhomogeneous spin chains delivering more insight into the structures of these models, as well as their limitations at wrapping order.
\end{abstract}

PACS numbers: $75.10 . \mathrm{Pq}, 02.30 . \mathrm{Ik}, 11.25 . \mathrm{Tq}$

\section{Contents}

List of symbols $\quad 2$

1. Introduction and overview $\quad 3$

2. Integrable spin chain models $\quad 6$

2.1. Integrable spin chains and local operators 6

2.2. Nearest neighbor models $\quad 8$

2.3. Long-range models 9

3. Algebra-preserving deformations 9

3.1. Generating equation 10

3.2. Local operators 11

3.3. Boost operators $\quad 12$

$\begin{array}{ll}\text { 3.4. Bilocal operators } & 15\end{array}$

$1751-8113 / 09 / 285205+58 \$ 30.00 \quad$ C 2009 IOP Publishing Ltd Printed in the UK 1 
3.5. Basis of charges 17

3.6. Symmetry generators 18

4. Geometry of the moduli space 19

4.1. Multi-parameter deformations $\quad 19$

4.2. Connection and curvature 20

4.3. Flatness 22

5. Interaction range $\quad 23$

5.1. First comparison to alg $\mathrm{gl}(\mathrm{K})$ chains 23

5.2. Boost connection at leading order $\quad 24$

5.3. Boost connection at higher orders 25

5.4. Bilocal connection at higher orders $\quad 26$

$\begin{array}{ll}\text { 5.5. Flatness } & 27\end{array}$

5.6. Summary 28

5.7. Properties of deformations 28

6. Long-range Bethe ansatz $\quad 31$

6.1. Ferromagnetic vacuum 31

6.2. One-magnon states and dispersion relations $(\alpha) \quad 32$

6.3. Two-magnon states and scattering $(\beta) \quad 33$

6.4. Basis of charges $(\gamma) \quad 35$

6.5. Higher rank and multiple magnons 36

6.6. Closed chain Bethe equations $\quad 36$

7. Alternating spin chains $\quad 38$

8. Inhomogeneous versus long-range spin chains 45

8.1. Bethe equations 45

8.2. Charge eigenvalues 46

8.3. Charge operators $\quad 47$

8.4. Beyond wrapping $\quad 49$

9. Conclusions and outlook $\quad 50$

Appendix A. Cancellation of longest terms $\quad 52$

Appendix B. Reading off the charges $\quad 53$

References $\quad 56$

\section{List of symbols}

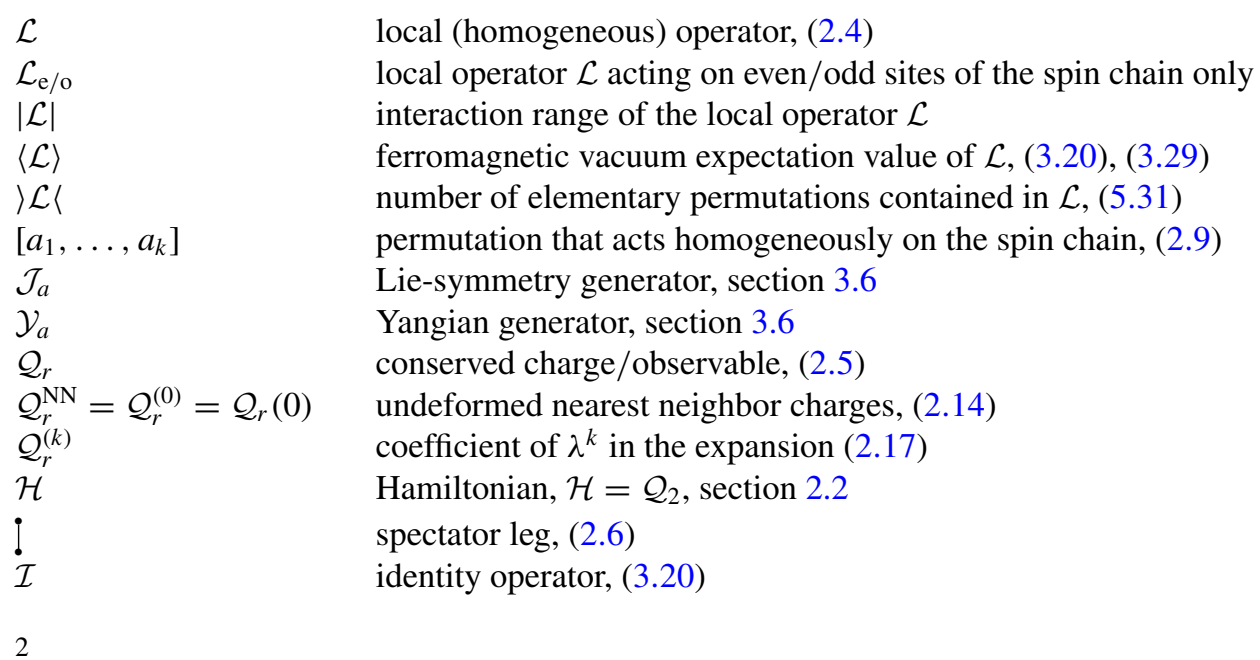




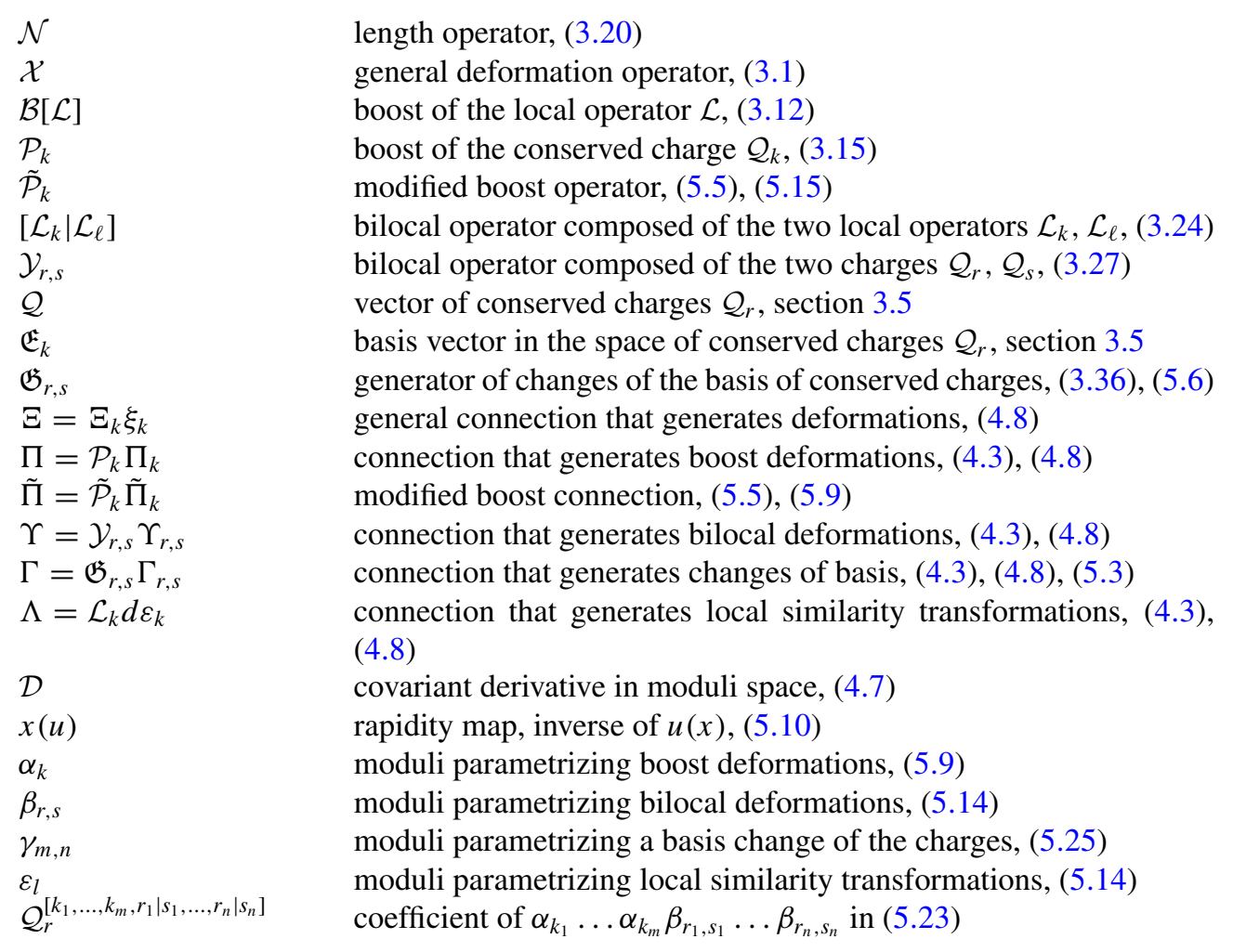

\section{Introduction and overview}

Much progress has been made in the last few years toward a verification of the proposed duality [1] between planar $\mathcal{N}=4$ super Yang-Mills theory (SYM) and type IIB string theory on $\mathrm{AdS}_{5} \times \mathrm{S}^{5}$. In this venture, integrability turned out to be an important feature of the spin chain structure underlying both theories in the planar limit [2-5]. A novel class of longrange spin chains had to be considered to reflect the complexity of the proposed dual theories $[3,5,6]$. A similar class of integrable chains [7] appears to be the key to rapid progress within the recently conjectured duality [8] between $\mathcal{N}=6$ superconformal Chern-Simons theory (SCS) and type IIA string theory on $\mathrm{AdS}_{4} \times \mathbb{C P}^{3}$.

It is remarkable that the only well-studied examples of long-range spin chains come from a completely different branch of physics: the condensed matter models described by HaldaneShastry [9] and Inozemtsev [10] incorporate interactions of well separated as opposed to nearest neighbor sites of the chain. These interactions, however, involve only two spins at a time, while the more general long-range operators arising from gauge/string duality act on several sites at the same time. Extensions of the Haldane-Shastry chain to multi-site interactions were also studied [11]. Their nature, however, is different from the gauge/string theory inspired interactions. Nevertheless, intersections among the condensed matter and high-energy spin chain models are inherent and give rise to a fruitful overlap of interests.

In this work we investigate integrable long-range spin chain models from a general point of view. Our class of models includes as special cases the above-mentioned spin chains. The structure of the models can be motivated best by its origin in gauge theory: trace operators 
providing a basis for all local gauge-invariant operators of $\mathcal{N}=4$ super Yang-Mills theory, are mapped to spin chain states, e.g.

$$
\operatorname{Tr}\left[\phi_{1} \phi_{2} \phi_{2} \phi_{1} \phi_{1} \phi_{2}\right] \rightarrow|\uparrow \downarrow \downarrow \uparrow \uparrow \downarrow\rangle .
$$

That is, fields transforming under some spacetime symmetry are mapped to spins transforming under the same symmetry algebra. It was shown that the one-loop Hamiltonian of planar $\mathcal{N}=4 \mathrm{SYM}$ is equivalent to a nearest neighbor spin chain Hamiltonian [2, 12]. The spectral problem of the gauge theory therefore becomes equivalent to the spectral problem of the spin chain. Excitingly the Hamiltonian turns out to be integrable [2, 5] leading to remarkable simplifications in the computation of the spectrum. Similar observations were made for $\mathcal{N}=6 \mathrm{SCS}$ theory [7, 13]. For several subsectors of $\mathcal{N}=4 \mathrm{SYM}$, the correspondence was generalized to higher loop orders $[3,14,15]$. Higher powers of the 't Hooft coupling constant $\lambda$ arising from vertices in planar Feynman diagrams indicate an increasing interaction range of the spin chain Hamiltonian:

$$
\mathcal{H}(\lambda)=\sum_{0}^{9}+\lambda \prod_{0}^{9}+\lambda^{2}
$$

While the nearest neighbor Hamiltonian $\mathcal{H}(\lambda=0)$ only acts on two neighboring spin sites at a time, a contribution at order $\lambda^{k}$ is allowed to have interactions among at most $k+2$ spins. For finite coupling $\lambda$, the Hamiltonian would actually be of infinite range which led to the notion of long-range spin chains. This class of chains can be considered as a long-range deformation of the prime example of a spin chain, the Heisenberg model [16].

Generally, the integrability of a spin chain system manifests itself in the existence of an infinite tower of local conserved charges $\mathcal{Q}_{r}$, the first of which is usually the Hamiltonian $\mathcal{Q}_{2}(\lambda)=\mathcal{H}(\lambda)$

$$
\left[\mathcal{Q}_{r}(\lambda), \mathcal{Q}_{s}(\lambda)\right]=0, \quad r, s=2,3, \ldots
$$

In the magnon excitation picture around the ferromagnetic vacuum this implies the factorization of multi-magnon scattering into two-magnon scattering. Admissible eigenstates of the Hamiltonian on a finite periodic chain of length $N$ can then be constructed by means of the Bethe ansatz [17], which was first introduced in 1931 for the Heisenberg model with $\mathfrak{s u}(2)$ symmetry [16]. It represents a periodicity condition on the magnon momenta $p_{k}$ or rapidities $u_{k}=\frac{1}{2} \cot \left(\frac{1}{2} p_{k}\right)$, in terms of the two-magnon scattering factor $S^{\mathrm{NN}}\left(u_{k}-u_{j}\right)=$ $\left(u_{k}-u_{j}+\mathrm{i}\right) /\left(u_{k}-u_{j}-\mathrm{i}\right)$ which altogether quantizes the spectrum

$$
\frac{\left(u_{k}+\frac{\mathrm{i}}{2}\right)^{N}}{\left(u_{k}-\frac{\mathrm{i}}{2}\right)^{N}}=\prod_{\substack{j=1 \\ j \neq k}}^{M} S^{\mathrm{NN}}\left(u_{k}-u_{j}\right), \quad k=1, \ldots, M .
$$

Similar to the spin chain charges, this ansatz was perturbatively generalized to the class of longrange chains by deformations in the coupling constant $[6,18]$. The two-magnon scattering factor was deformed by the so-called dressing phase $\theta\left(u_{k}, u_{j} ; \lambda\right)$ and a rapidity map $x(u ; \lambda)$ deforming the term $u_{k} \pm \frac{i}{2}$ was introduced. The resulting long-range $\mathfrak{s u}(2)$ equations read

$$
\frac{x\left(u_{k}+\frac{\mathrm{i}}{2}\right)^{N}}{x\left(u_{k}-\frac{\mathrm{i}}{2}\right)^{N}}=\prod_{\substack{j=1 \\ j \neq k}}^{M} S^{\mathrm{NN}}\left(u_{k}-u_{j}\right) \exp \left(2 \mathrm{i} \theta\left(u_{k}, u_{j}\right)\right), \quad k=1, \ldots, M .
$$

They were later extended to the complete spectrum of $\mathcal{N}=4$ SYM [19] and $\mathcal{N}=6$ SCS [20]. These asymptotic Bethe equations describe the spectrum of the Hamiltonian for long chains. 
For finite chains the interaction range of the Hamiltonian necessarily exceeds the length of the spin chain at some order of the coupling $\lambda$. Beyond this order it is not known if or how the Bethe ansatz can be modified to incorporate these so-called wrapping interactions. For recent progress on the understanding of the wrapping problem in the special context of gauge/string duality see [21-23].

Recently, a first step was taken toward a more general exploration of long-range integrable spin chains. In [24] a study of the $\mathfrak{g l}(K)$ chain incorporating the gauge theory $\mathfrak{s u}(2)$ sector as a special case was presented: starting with a generic ansatz for the first two integrable charges and imposing (1.3) on this ansatz, the form of the charges was determined up to some perturbative order in the coupling. The moduli space for closed chains was found to be characterized by four different types of parameters: the parameters $\alpha_{k}$ govern the degrees of freedom corresponding to deformations of the dispersion relation by means of the rapidity map $x(u)$. The parameters $\beta_{r, s}$ account for deformations by means of the dressing phase $\theta\left(u_{k}, u_{j}\right)$, cf (1.5). The additional moduli $\gamma_{m, n}$ and $\varepsilon_{l}$ correspond to linear combinations of commuting charges and similarity transformations of the integrable system, respectively, and have no impact on the spectrum. The most general integrable Hamiltonian $\mathcal{H}=\mathcal{H}\left(\alpha_{k}, \beta_{r, s}, \gamma_{r, s}, \varepsilon_{k}\right)$ then takes the schematic form

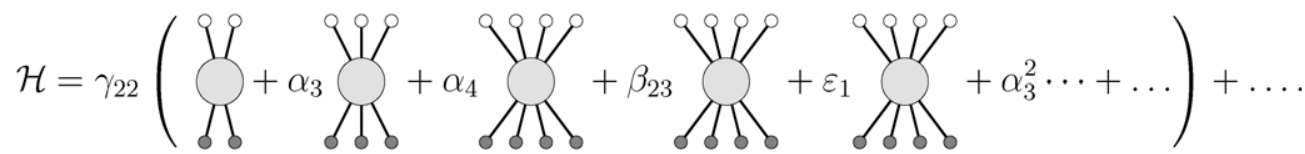

Generalizing the long-range gauge theory model to this chain, the single deformation parameter $\lambda$ was replaced by four different sets of moduli with different physical interpretations. A certain choice of the new parameters as functions of $\lambda$ results in the special expansion (1.2). While the assumption of integrability of the $\mathfrak{g l}(K)$ chain in [24] was based on the existence of two commuting charges, a perturbative proof for its integrability up to $\mathcal{O}\left(\lambda^{3}\right)$ was later presented in [25] by the construction of a Yangian representation. However, a formal setting for the understanding of the all-order integrable long-range spin chain is still lacking. A rigorous mathematical construction might provide a scenery for the understanding of wrapping effects in a general context.

In this work we develop a framework for the construction of long-range integrable spin chains of arbitrary Lie (super)algebra symmetry. We present a recursion relation for the charges $\mathcal{Q}_{t}\left(\alpha_{k}, \beta_{r, s}, \gamma_{r, s}, \varepsilon_{k}\right)$ whose solutions are manifestly integrable to all orders in the deformation parameters and cover the whole moduli space explored in [24]. This proves the all-order existence of an integrable long-range model on infinite spin chains. Furthermore the recursion allows the explicit construction of the integrable charge operators. The four different types of moduli are related to four different types of deformation operators. Analyzing their impact on short-range spin chains explains the emergence of the dressing phase and the rapidity map in the long-range Bethe equations. This work is an extension of the considerations sketched in the paper [26]. The paper is structured as follows:

Section 2: We introduce the framework of integrable long-range spin chains of infinite extent.

Section 3: A recursion relation is defined which induces a set of manifestly integrable longrange charges as deformations of a short-range system. Four different kinds of deformation generators corresponding to the four different moduli discussed above are presented. 
Section 4: The geometry of the moduli space is analyzed. We investigate the curvature associated with the generating equation and derive flatness conditions for the deformations.

Section 5: We present a parametrization that minimizes the interaction range of the charges at each order of the deformation moduli and at the same time renders the space of deformed charges flat. This parametrization yields integrable charges as they occur in the gauge/string correspondence.

Section 6: It is demonstrated how the four different types of generators deform the nearest neighbor Bethe ansatz. Most notably, the appearance of the rapidity map as well as the dressing phase is explained by classes of so-called boost and bilocal operators, respectively. The result is given by the well-known form of the asymptotic Bethe equations for finite periodic chains.

Section 7: We demonstrate how to apply the recursion relation to alternating spin chains. The alternating $\mathfrak{s u}(2) \times \mathfrak{s u}(2)$ long-range Bethe equations as well as the first orders of the $\mathfrak{g l}\left(K_{\mathrm{e}}\right) \times \mathfrak{g l}\left(K_{\mathrm{o}}\right)$ long-range Hamiltonian are explicitly given.

Section 8: Finally and somewhat outside the main theme of this paper we present a map relating homogeneous long-range chains without dressing phase to inhomogeneous chains. The latter can be defined consistently even beyond wrapping order, however, in almost arbitrary ways.

Most parts of the paper focus on deformations of operators on spin chains with infinite extent. In section 6, parts of sections 7 and 8, we consider implications of these deformations on finite chains.

\section{Integrable spin chain models}

\subsection{Integrable spin chains and local operators}

A spin chain is a physical model based on a Hilbert space $\mathbb{H}$ which is a tensor product of identical vector spaces $\mathbb{V}_{a}=\mathbb{V}$ :

$$
\mathbb{H}=\cdots \otimes \mathbb{V}_{a} \otimes \mathbb{V}_{a+1} \otimes \mathbb{V}_{a+2} \otimes \cdots, \quad \mathbb{V}_{a}=\mathbb{V}
$$

The vector spaces $\mathbb{V}_{a}$ are labeled by consecutive integers $a$ describing the position along the chain; neighboring sites of the chain have adjacent integer positions. The chain can be finite and have open or periodic boundary conditions. Alternatively, it can have infinite extent which is the case we shall consider in the first half of this paper. A basis of the Hilbert space $\mathbb{H}$ is given by states for which the spin at each site $a$ has a definite orientation $v_{a}$ being a basis vector of $\mathbb{V}$ :

$$
\left|\ldots, v_{a}, v_{a+1}, v_{a+2}, \ldots\right\rangle \in \mathbb{H} .
$$

Typically, there is also a Lie symmetry algebra $\mathfrak{g}$ which can be represented on the vector space $\mathbb{V}$, i.e. $\mathbb{V}$ can be considered as a (generalized) spin of $\mathfrak{g}$.

The physical model is furthermore defined by a set of observables which are linear operators $\mathcal{A}$ acting on the Hilbert space

$$
\mathcal{A}: \mathbb{H} \rightarrow \mathbb{H} \text {. }
$$

Typically, the operators have some well-defined transformation properties under the symmetry algebra $\mathfrak{g}$, i.e. they may be invariant or transform in a certain representation. 


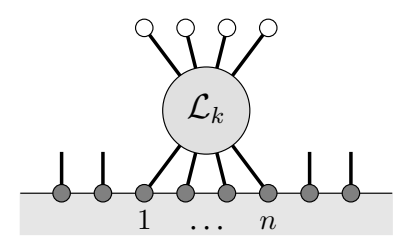

Figure 1. A local operator $\mathcal{L}_{k}$ acting on a spin chain. Its position on the chain is summed over, see (2.4).

We are mainly interested in operators that act locally and homogeneously on the spin chain and are invariant under the symmetry $\mathfrak{g}$. We call these simply local operators and denote them by

$\mathcal{L}_{k}:=\sum_{a} \mathcal{L}_{k}(a), \quad \mathcal{L}_{k}(a): \mathbb{V}_{a} \otimes \cdots \otimes \mathbb{V}_{a+n-1} \rightarrow \mathbb{V}_{a} \otimes \cdots \otimes \mathbb{V}_{a+n-1}$.

Here $\mathcal{L}_{k}(a)$ is some linear operator which acts on several consecutive spins starting with site $a$, cf figure 1. The number $n$ of interacting sites is called the interaction range of the operator $\mathcal{L}_{k}$ and will be denoted by $\left|\mathcal{L}_{k}\right|=n$. These operators furnish the basic framework for the following considerations.

An integrable spin chain model is defined by an infinite tower of commuting charges acting locally and homogeneously on an infinite chain

$$
\left[\mathcal{Q}_{r}, \mathcal{Q}_{s}\right]=0, \quad r, s=2,3, \ldots
$$

Integrability can also be defined for finite chains, cf sections 6 and 8, but for the time being we shall restrict ourselves to infinite chains. Integrable spin chains are generally based on some Lie algebra $\mathfrak{g}$ or a quantum deformation thereof. The algebra is a symmetry of the charges $\mathcal{Q}_{r}$. In fact, the symmetry typically extends to an infinite-dimensional algebra of Yangian or quantum-affine type [27, 28].

Local operator identifications. The definition of homogeneous local operators in (2.4) allows for identifications of certain operators on infinite chains. For a local operator $\mathcal{L}_{k}$ of range $n$ define the local operators $\mathfrak{j} \mathcal{L}_{k}$ and $\mathcal{L}_{k} \mathfrak{j}$ of range $n+1$ as follows:

$$
\left\lceil\mathcal{L}_{k}(a):=\mathcal{I}(a) \otimes \mathcal{L}_{k}(a+1), \quad \mathcal{L}_{k} !(a):=\mathcal{L}_{k}(a) \otimes \mathcal{I}(a+n),\right.
$$

where $\mathcal{I}(a)$ is the identity operator acting on site $a$. The additional identity operators are called spectator legs of the local operator because their action is trivial. Clearly, all three operators are equivalent after the position $a$ is summed over in (2.4), see also figure 2,

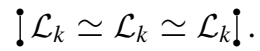

Note that on finite chains these operators are equivalent only up to boundary terms.

Example. For illustration purposes we will make a specific choice of the symmetry algebra and its representation at certain points of the text. We will choose the fundamental representation of $\mathfrak{g}=\mathfrak{g l}(K)$ on the vector space $\mathbb{V}=\mathbb{C}^{K}$. Spin orientations can then simply be denoted by numbers corresponding to one of the $K$ directions, e.g. a basis vector of the Hilbert space is given by

$$
|\ldots, 3,2,4,1,2,4, \ldots\rangle \text {. }
$$




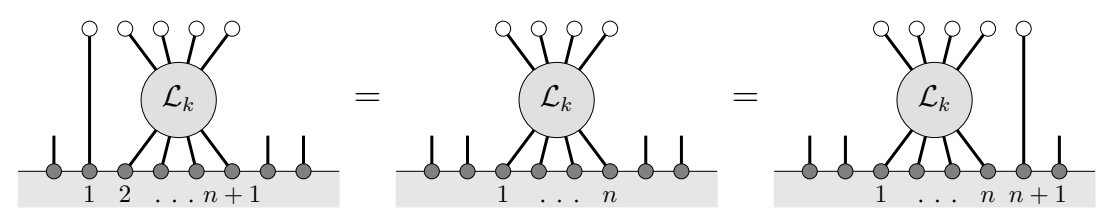

Figure 2. On a spin chain without boundaries, local homogeneous operators that differ only by spectator legs can be identified. The position on the spin chain at which the operators act is implicitly summed over.

$$
\mathcal{Q}_{2}^{\mathrm{NN}} \stackrel{\mathcal{B}\left[\mathcal{Q}_{2}^{\mathrm{NN}}\right]}{\longrightarrow} \mathcal{Q}_{3}^{\mathrm{NN}} \stackrel{\mathcal{B}\left[\mathcal{Q}_{2}^{\mathrm{NN}}\right]}{\longrightarrow} \mathcal{Q}_{4}^{\mathrm{NN}} \stackrel{\mathcal{B}\left[\mathcal{Q}_{2}^{\mathrm{NN}}\right]}{\longrightarrow} \mathcal{Q}_{5}^{\mathrm{NN}} \stackrel{\mathcal{B}\left[\mathcal{Q}_{2}^{\mathrm{NN}}\right]}{\longrightarrow} \cdots
$$

Figure 3. The short-range charges are generated iteratively by the so-called boost operator $\mathcal{B}\left[\mathcal{Q}_{2}^{\mathrm{NN}}\right]$, cf (2.14) and [29].

For this specific choice of algebra and representation, a basis of invariant local operators $\mathcal{L}_{\pi}$ consists of permutations $\pi$ of nearby spins: a permutation $\pi \in S_{n}$ of range $n$, mapping the spin sites $(a+1, a+2, \ldots, a+n)$ to the sites $(a+\pi(1), a+\pi(2), \ldots, a+\pi(n))$, is denoted by

$$
\mathcal{L}_{\pi}(a+1)=[\pi(1), \pi(2), \ldots, \pi(n)]_{a+1} .
$$

The building blocks for $\mathfrak{g l}(K)$-invariant operators are then given by permutation symbols acting homogeneously on the whole chain

$$
\mathcal{L}_{\pi}=[\pi(1), \pi(2), \ldots, \pi(n)]=\sum_{a}[\pi(1), \pi(2), \ldots, \pi(n)]_{a} .
$$

As an example, the operator $[2,1]=\sum_{a} \mathcal{P}_{a, a+1}$ sums over all pairs of nearest neighbors, $(a, a+1)$, and permutes them $\left(\mathcal{P}_{a, a+1}\right)$

$[2,1]|\ldots, 1,2,3, \ldots\rangle=\cdots+|\ldots, 2,1,3, \ldots\rangle+|\ldots, 1,3,2, \ldots\rangle+\cdots$.

Adding spectator legs to $[2,1]$ according to (2.6) yields the following two operators:

$$
[[2,1]=[1,3,2], \quad[2,1]\rfloor=[2,1,3] .
$$

Note that $\mathfrak{g}=\mathfrak{g l}(K)$ only serves as an illustrative example for the following ideas, most of which are valid for a generic Lie symmetry algebra $\mathfrak{g}$ and arbitrary spin representations.

\subsection{Nearest neighbor models}

An ordinary nearest neighbor spin chain is characterized by a Hamiltonian $\mathcal{H}=\mathcal{Q}_{2}^{\mathrm{NN}}$, which acts on two adjacent sites at a time only. The set of commuting charges can then be ordered by their interaction range starting with the Hamiltonian. The charges are given by some linear combination of local operators

$$
\mathcal{Q}_{r}^{\mathrm{NN}}=\sum_{k} c_{r, k} \mathcal{L}_{k}
$$

The coefficients $c_{r, k}$ can be uniquely fixed by a suitable normalization condition but only modulo identification of spectator legs (2.7).

Note that there exists an iterative definition of the commuting charges [29]: based on the nearest neighbor Hamiltonian $\mathcal{Q}_{2}^{\mathrm{NN}}$ one can define a so-called boost operator $\mathcal{B}\left[\mathcal{Q}_{2}^{\mathrm{NN}}\right]$ such that the integrable system is generated by a single equation (cf figure 3 )

$$
\mathcal{Q}_{r+1}^{\mathrm{NN}}=-\frac{\mathrm{i}}{r}\left[\mathcal{B}\left[\mathcal{Q}_{2}^{\mathrm{NN}}\right], \mathcal{Q}_{r}^{\mathrm{NN}}\right] .
$$


The precise definition of $\mathcal{B}[\cdot]$ will be discussed in section 3.3. The interaction range of the charges following from this iteration relation reads for a nearest neighbor Hamiltonian with $\left|\mathcal{Q}_{2}^{\mathrm{NN}}\right|=2$ :

$$
\left|\mathcal{Q}_{r}^{\mathrm{NN}}\right|=(r-1)\left|\mathcal{Q}_{2}^{\mathrm{NN}}\right|-(r-2)=r .
$$

Example. For $\mathfrak{g}=\mathfrak{g l}(K)$ the first few charges take the form, see, e.g. [24]

$$
\begin{aligned}
\mathcal{Q}_{2}^{\mathrm{NN}} & =[1]-[2,1], \\
\mathcal{Q}_{3}^{\mathrm{NN}} & =\frac{\mathrm{i}}{2}([3,1,2]-[2,3,1]), \\
\mathcal{Q}_{4}^{\mathrm{NN}} & =\frac{1}{3}(-[1]+2[2,1]-[3,2,1]+[2,3,4,1] \\
& \quad-[2,4,1,3]-[3,1,4,2]+[4,1,2,3]) .
\end{aligned}
$$

\subsection{Long-range models}

Perturbatively long-ranged spin chains are formally defined as deformations of the above nearest neighbor chains. The nearest neighbor charges $\mathcal{Q}_{r}^{\mathrm{NN}}$ are taken to be the leading order $\mathcal{Q}_{r}^{(0)}$ in a formal power series

$$
\mathcal{Q}_{r}(\lambda)=\mathcal{Q}_{r}^{(0)}+\lambda \mathcal{Q}_{r}^{(1)}+\lambda^{2} \mathcal{Q}_{r}^{(2)}+\mathcal{O}\left(\lambda^{3}\right), \quad \mathcal{Q}_{r}^{(0)}=\mathcal{Q}_{r}^{\mathrm{NN}},
$$

such that the interaction range of the charges grows linearly with the perturbative order in $\lambda$, e.g. $\left|\mathcal{Q}_{r}^{(k)}\right|=r+k$ [3]. Hence, assuming that $\lambda$ can take finite values, the charges $\mathcal{Q}_{r}(\lambda)$ would be of infinite (i.e. long) range.

The long-range charges can still be written as linear combinations of local operators

$$
\mathcal{Q}_{r}(\lambda)=\sum_{k} c_{r, k}(\lambda) \mathcal{L}_{k}
$$

but now with coefficients $c_{r, k}(\lambda)$ which are formal power series in $\lambda$ starting at a certain order, e.g. $c_{r, k}(\lambda)=\mathcal{O}\left(\lambda^{\left|\mathcal{L}_{k}\right|-r}\right)$. The charges have to obey the integrability condition (2.5) order by order in $\lambda$.

It is the aim of this paper to present an equation (similar to the recursion relation (2.14)) that generates the long-range system through deformations of the nearest neighbor charges. The resulting long-range spin chain model will be manifestly integrable.

Example. For fundamental $\mathfrak{g}=\mathfrak{g l}(K)$ chains, the charges take the form [24]

$$
\begin{gathered}
\mathcal{Q}_{2}(\lambda)=[1]-[2,1]+\alpha_{3} \lambda(-3[1]+4[2,1]-[3,2,1])+\mathcal{O}\left(\lambda^{2}\right), \\
\mathcal{Q}_{3}(\lambda)=\frac{1}{2}([3,1,2]-[2,3,1])+\frac{i}{2} \alpha_{3} \lambda(6[2,3,1]-6[3,1,2]+[4,1,3,2] \\
+[4,2,1,3]-[2,4,3,1]-[3,2,4,1])+\mathcal{O}\left(\lambda^{2}\right) .
\end{gathered}
$$

Note that in contrast to the nearest neighbor chain not all coefficients $c_{r, k}(\lambda)$ are fixed by integrability but some free parameters $\xi_{k}=\left\{\alpha_{3}, \ldots\right\}$ remain. The latter are thus moduli of the long-range integrable system.

\section{Algebra-preserving deformations}

In the following a general mechanism for the construction of long-range spin chains is presented. The key idea is a differential equation that generates long-range charges as deformations of some short-range (e.g. nearest neighbor) charges. We present transformations 
of the integrable charges of an infinite short-range spin chain that do not leave the space of local, homogeneous operators and preserve integrability. Later (section 6), we study finite chains, i.e. the impact of the presented deformations on the boundary conditions.

The construction is applicable to short-range spin chains, which usually have a Lie symmetry algebra $\mathfrak{g}$ under which the integrable charges are invariant. In the present work we make the assumption that the representation of $\mathfrak{g}$ on the spin chain remains undeformed. We shall illustrate the various deformations by means of examples for the specific case of $\mathfrak{g}=\mathfrak{g l}(K)$ with spins transforming in the fundamental representation.

\subsection{Generating equation}

Consider an algebra of charges $\mathcal{Q}_{r}(0)$ (not necessarily abelian) and a deformation $\mathcal{Q}_{r}(\lambda)$ which obeys the differential equation

$$
\frac{\mathrm{d}}{\mathrm{d} \lambda} \mathcal{Q}_{r}(\lambda)=\mathrm{i}\left[\mathcal{X}(\lambda) \mathcal{Q}_{r}(\lambda)\right]
$$

Here, $\mathcal{X}(\lambda)$ is some yet-to-be specified operator that has well-defined commutation relations with the charges $\mathcal{Q}_{r}(\lambda)$ for all $\lambda$, in particular in the sense of a formal power series in $\lambda$.

The deformation (3.1) leaves the algebra of charges $\mathcal{Q}_{r}(\lambda)$ unchanged: It implies by the Jacobi identity

$$
\frac{\mathrm{d}}{\mathrm{d} \lambda}\left[\mathcal{Q}_{r}(\lambda) \mathcal{Q}_{s}(\lambda)\right]=\mathrm{i}\left[\mathcal{X}(\lambda),\left[\mathcal{Q}_{r}(\lambda) \mathcal{Q}_{s}(\lambda)\right]\right]
$$

For a generic algebra of independent operators $\mathcal{Q}_{r}$, this shows that the structure constants $f_{\text {rst }}$ do not change under the deformation

$$
\left[\mathcal{Q}_{r}(\lambda), \mathcal{Q}_{s}(\lambda)\right]=f_{\mathrm{rst}} \mathcal{Q}_{t}(\lambda), \quad \frac{\mathrm{d}}{\mathrm{d} \lambda} f_{\mathrm{rst}}=0
$$

In particular, if the algebra of the initial charges $\mathcal{Q}_{r}(0)$ is abelian, $f_{\text {rst }}=0$, also the algebra of the deformed charges defined by (3.1) is abelian for all $\lambda$ and any $\mathcal{X}(\lambda)$

$$
\left[\mathcal{Q}_{r}(0), \mathcal{Q}_{s}(0)\right]=0 \quad \Longrightarrow \quad\left[\mathcal{Q}_{r}(\lambda), \mathcal{Q}_{s}(\lambda)\right]=0
$$

Thus the differential equation (3.1) preserves the existence of commuting charges which represents the most important property (2.5) of any integrable system. However, for integrable spin chains including our class of models one requires stronger properties: the charges $\mathcal{Q}_{r}(\lambda)$ must act locally and homogeneously (2.4) on the spin chain. Hence, if the deformation (3.1) is to describe such models, the resulting charges $\mathcal{Q}_{r}(\lambda)$ must not violate these properties. That is, the operators $\mathcal{X}(\lambda)$ must be chosen such that

$$
\left[\mathcal{X}(\lambda), \mathcal{Q}_{r}(\lambda)\right] \quad \text { is local and homogeneous }
$$

for all $\lambda$. The possible choices for $\mathcal{X}(\lambda)$ that satisfy these requirements are discussed in the following subsections.

Before we continue, we will construct a perturbative solution to the generating equation (3.1). We first integrate it,

$$
\mathcal{Q}_{r}(\lambda)=\mathcal{Q}_{r}(0)+\int_{0}^{\lambda} \mathrm{d} \lambda^{\prime} \mathrm{i}\left[\mathcal{X}\left(\lambda^{\prime}\right), \mathcal{Q}_{r}\left(\lambda^{\prime}\right)\right] .
$$


Expansion into a power series in $\lambda$ then straightforwardly yields

$$
\begin{aligned}
& \mathcal{Q}_{r}(\lambda)=\mathcal{Q}_{r}^{(0)}+\lambda \mathcal{Q}_{r}^{(1)}+\lambda^{2} \mathcal{Q}_{r}^{(2)}+\lambda^{3} \mathcal{Q}_{r}^{(3)}+\cdots, \\
& \mathcal{X}(\lambda)=\mathcal{X}^{(0)}+\lambda \mathcal{X}^{(1)}+\lambda^{2} \mathcal{X}^{(2)}+\lambda^{3} \mathcal{X}^{(3)}+\cdots, \\
& \mathcal{Q}_{r}^{(1)}=\mathrm{i}\left[\mathcal{X}^{(0)}, \mathcal{Q}_{r}^{(0)}\right], \\
& \mathcal{Q}_{r}^{(2)}=\frac{\mathrm{i}}{2}\left[\mathcal{X}^{(1)}, \mathcal{Q}_{r}^{(0)}\right]+\frac{\mathrm{i}}{2}\left[\mathcal{X}^{(0)}, \mathcal{Q}_{r}^{(1)}\right], \\
& \mathcal{Q}_{r}^{(3)}=\cdots .
\end{aligned}
$$

This shows that the higher orders of the charges are completely determined through the lower orders by iteration. The generating equation is thus a recursion relation for the construction of an integrable system.

\subsection{Local operators}

The commutator of two local operators is again local and homogeneous. Hence any local operator $\mathcal{L}_{l}$ results in an admissible deformation $\mathcal{X}=\mathcal{L}_{l}(3.1)$ of the charges

$$
\frac{\mathrm{d}}{\mathrm{d} \lambda} \mathcal{Q}_{r}(\lambda)=\mathrm{i}\left[\mathcal{L}_{l}, \mathcal{Q}_{r}(\lambda)\right]
$$

Note that this deformation can be integrated exactly

$$
\begin{aligned}
\mathcal{Q}_{r}(\lambda) & =\exp \left(+\mathrm{i} \lambda \mathcal{L}_{l}\right) \mathcal{Q}_{r}(0) \exp \left(-\mathrm{i} \lambda \mathcal{L}_{l}\right) \\
& =\left(1+\mathrm{i} \lambda \mathcal{L}_{l}\right) \mathcal{Q}_{r}^{(0)}\left(1-\mathrm{i} \lambda \mathcal{L}_{l}\right)+\mathcal{O}\left(\lambda^{2}\right) \\
& =\mathcal{Q}_{r}^{(0)}+\mathrm{i} \lambda\left[\mathcal{L}_{l}, \mathcal{Q}_{r}^{(0)}\right]+\mathcal{O}\left(\lambda^{2}\right) .
\end{aligned}
$$

It thus merely constitutes a similarity transformation of the charges $\mathcal{Q}_{r}$ by the operator $\exp \left(i \lambda \mathcal{L}_{l}\right)$. Importantly, as we shall see in section 6 , this deformation does not change any of the quantities we are ultimately interested in. In the following we shall therefore disregard transformations by local operators. In any case, one can easily reintroduce them at the very end through the similarity transformation (3.9).

Degrees of freedom. When counting all degrees of freedom of the system, i.e. the total number of possible deformation operators $\mathcal{X}$ (of a given maximum range), it has to be taken into account that the conserved charges $\mathcal{X}=\mathcal{Q}_{r}$ generate trivial deformations due to the integrability condition (2.5). Moreover, on a spin chain without boundaries, local operators that differ by spectator legs only (2.6), must be identified, cf (2.7) and figure 2.

Example. As an example of a deformation of the type (3.8), consider the $\mathfrak{g l}(K)$ spin chain. As mentioned above (2.9), the only invariant operators on this spin chain are permutations

$$
\mathcal{L}_{k}=[\pi(1), \pi(2), \ldots, \pi(n)],
$$

which act homogeneously on the chain. Deforming the nearest neighbor Hamiltonian $\mathcal{Q}_{2}^{(0)}=[1]-[2,1]$ of this chain by the local homogeneous operator $\mathcal{L}=[3,2,1]$ yields the deformed Hamiltonian

$$
\begin{aligned}
\mathcal{Q}_{2} & =\mathcal{Q}_{2}^{(0)}+\lambda \mathcal{Q}_{2}^{(1)}+\mathcal{O}\left(\lambda^{2}\right) \\
\mathcal{Q}_{2}^{(1)} & =\mathrm{i}\left[\mathcal{L}, \mathcal{Q}_{2}^{(0)}\right]=\mathrm{i}[[3,2,1],[2,1]] \\
& =\mathrm{i}([4,2,1,3]-[3,2,4,1]+[2,4,3,1]-[4,1,3,2]) .
\end{aligned}
$$




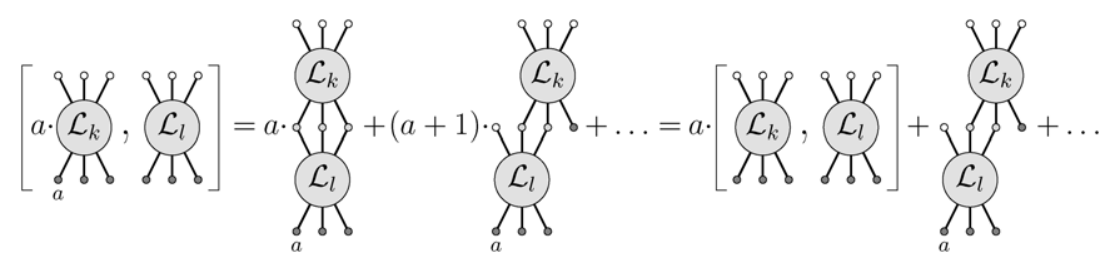

Figure 4. Graphical representation of the commutator between a boost operator $\mathcal{B}\left[\mathcal{L}_{k}\right]$ and a local operator $\mathcal{L}_{l}$. The contributions combine into the boost of the commutator $\left[\mathcal{L}_{k}, \mathcal{L}_{l}\right]$ between the two local operators plus local terms, cf (3.13), (3.14). When the local operators commute, only the local terms remain.

\subsection{Boost operators}

As mentioned above (2.14), it is well known [29] that a set of mutually commuting charges $\mathcal{Q}_{r}^{(0)}$ can be generated iteratively, starting from a nearest neighbor Hamiltonian $\mathcal{Q}_{2}^{(0)}$. The higher charges are constructed through commutation with the 'boosted' Hamiltonian $\mathcal{B}\left[\mathcal{Q}_{2}^{(0)}\right]$ : $\mathcal{Q}_{r+1}^{(0)} \sim\left[\mathcal{B}\left[\mathcal{Q}_{2}^{(0)}\right], \mathcal{Q}_{r}^{(0)}\right]$. We define the boost $\mathcal{B}\left[\mathcal{L}_{k}\right]$ of a local operator $\mathcal{L}_{k}$ as

$$
\mathcal{L}_{k}=\sum_{a} \mathcal{L}_{k}(a) \quad \Longrightarrow \quad \mathcal{B}\left[\mathcal{L}_{k}\right]:=\sum_{a} a \mathcal{L}_{k}(a),
$$

where the local operator $\mathcal{L}_{k}(a)$ acts on a set of adjacent spins, starting at site $a$. Boost operators defined in this way act locally, but inhomogeneously on the spin chain. For the purpose of a concise notation, we shall reserve the term local operator for local homogeneous operators; local inhomogeneous operators will be called boost operators.

In general, the commutator of a boost operator with a local operator yields a combination of boost and local operators (cf figure 4)

$$
\left[\mathcal{B}\left[\mathcal{L}_{k}\right], \mathcal{L}_{l}\right]=\mathcal{B}\left[\left[\mathcal{L}_{k}, \mathcal{L}_{l}\right]\right]+\mathcal{L}_{r}
$$

with $\mathcal{L}_{r}$ some local operator. However, if the underlying local operators commute, the commutator becomes local

$$
\left[\mathcal{L}_{k}, \mathcal{L}_{l}\right]=0 \quad \Longrightarrow \quad\left[\mathcal{B}\left[\mathcal{L}_{k}\right], \mathcal{L}_{l}\right]=\mathcal{L}_{r}
$$

Therefore, the boosts of the commuting charges themselves are operators that yield admissible deformations (3.1)

$$
\frac{\mathrm{d}}{\mathrm{d} \lambda} \mathcal{Q}_{r}(\lambda)=\mathrm{i}\left[\mathcal{B}\left[\mathcal{Q}_{k}(\lambda)\right], \mathcal{Q}_{r}(\lambda)\right], \quad k=3, \ldots, \infty,
$$

which result in charges $\mathcal{Q}_{r}(\lambda)$ that are homogeneous, as desired.

Note that the deformation operator $\mathcal{X}(\lambda)=\mathcal{B}\left[\mathcal{Q}_{k}(\lambda)\right]$ directly depends on the charges $\mathcal{Q}_{k}(\lambda)$ which are themselves the solution of the generating equation (3.1). This implies, in particular, that boost deformations are not simply exponential similarity transformations (3.9) of the undeformed charges; the dependence on the deformation parameter $\lambda$ is more involved. Nevertheless, the equation as well as its recursive solution (3.7) with $\mathcal{X}^{(n)}=\mathcal{B}\left[\mathcal{Q}_{k}^{(n)}\right]$ remains perfectly well defined.

Boost operator identifications. As illustrated in figure 2, local operators on an infinitely long chain that differ only by spectator legs can be identified (2.7). But according to (3.12), the corresponding boost operators would differ by a local operator. This is related to the fact that 

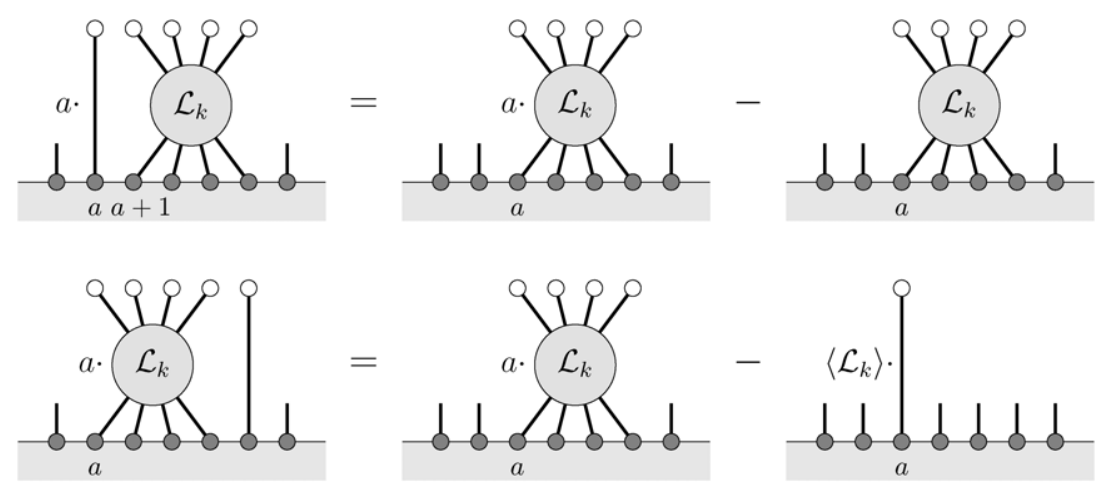

Figure 5. Illustration of the boost operator identifications (3.19) on an infinite chain. As in the previous pictures, the position of the local operators on the spin chain is summed over. The upper identification is evident by (3.16): putting a spectator leg to the left of the operator amounts to shifting the origin (site 0 ) of the chain by -1 . The subtraction of the length operator in the lower identity is required for a correct normalization and stems from the different action of the two boost operators at the right boundary of a finite chain. Using the symmetric regularization of the boost operator (3.17), the identifications become (3.20).

their definition depends on the location of the spin chain origin (site 0). Namely, shifting the origin by $n$ sites leads to a shift of $\mathcal{B}\left[\mathcal{L}_{k}\right]$ by the local operator $n \mathcal{L}_{k}$

$$
\mathcal{B}\left[\mathcal{L}_{k}\right] \rightarrow \mathcal{B}\left[\mathcal{L}_{k}\right]+n \mathcal{L}_{k}
$$

When deforming with a boosted charge $\mathcal{B}\left[\mathcal{Q}_{k}\right](3.15)$, the location of the spin chain origin is irrelevant because $\mathcal{Q}_{k}$ commutes with $\mathcal{Q}_{r}$. On an open chain of length $N$ whose leftmost site is labeled by $a=1$, we can thus regularize the boost operator (3.12) as

$$
\mathcal{B}\left[\mathcal{L}_{k}\right]=\sum_{a} a \mathcal{L}_{k}(a)-\frac{1}{2}\left(N-\left|\mathcal{L}_{k}\right|\right) \mathcal{L}_{k},
$$

where $\left|\mathcal{L}_{k}\right|$ denotes the range of the operator $\mathcal{L}_{k}$. This choice puts the origin in the middle of the finite chain such that $\mathcal{B}\left[\mathcal{L}_{k}\right]$ has exactly opposite parity of $\mathcal{L}_{k}$. On such an open chain, spectator legs on local operators yield boundary terms

$$
\left\lceil\mathcal{L}_{k}=\mathcal{L}_{k}-\mathcal{L}_{k}(1), \quad \mathcal{L}_{k} \grave{\bullet}=\mathcal{L}_{k}-\mathcal{L}_{k}\left(N-\left|\mathcal{L}_{k}\right|+1\right) .\right.
$$

Accordingly, boost operators are modified by spectator legs as (cf also figure 5)

$$
\begin{aligned}
& \sum_{a} a\left(\mathcal{L}_{k}\right)(a)=\sum_{a} a \mathcal{L}_{k}(a)-\mathcal{L}_{k}, \\
& \sum_{a} a\left(\mathcal{L}_{k} !\right)(a)=\sum_{a} a \mathcal{L}_{k}(a)-\left(N-\left|\mathcal{L}_{k}\right|+1\right) \mathcal{L}_{k}\left(N-\left|\mathcal{L}_{k}\right|+1\right) .
\end{aligned}
$$

In the limit $N \rightarrow \infty$, we can effectively replace the boundary operators $\mathcal{L}_{k}(1), \mathcal{L}_{k}\left(N-\left|\mathcal{L}_{k}\right|+1\right)$ by their expectation values $\left\langle\mathcal{L}_{k}\right\rangle$ on a ferromagnetic vacuum. Combining (3.18) and (3.19), we find that the relation between equivalent boost operators on an infinite chain is given by ${ }^{1}$

$$
\begin{aligned}
& \mathcal{B}\left[\mathcal{L}_{k}\right]=\mathcal{B}\left[\mathcal{L}_{k}\right]-\frac{1}{2}\left(\mathcal{L}_{k}-\left\langle\mathcal{L}_{k}\right\rangle \mathcal{N}\right), \\
& \mathcal{B}\left[\mathcal{L}_{k} !\right]=\mathcal{B}\left[\mathcal{L}_{k}\right]+\frac{1}{2}\left(\mathcal{L}_{k}-\left\langle\mathcal{L}_{k}\right\rangle \mathcal{N}\right),
\end{aligned} \quad \mathcal{N}:=\sum_{a} \mathcal{I}(a) .
$$

1 Without the regularization (3.17), we would have arrived at similar, but less symmetric identifications. For an elementary permutation $\mathcal{L}_{\pi}$ without a prefactor on a $\mathfrak{g l}(K)$ chain, $\left\langle\mathcal{L}_{\pi}\right\rangle=1$. 
Here, the symbol $\mathcal{I}(a)$ denotes the identity operator acting at spin site $a$ and $\mathcal{N}$ is thus the operator which measures the length of the chain. The combination $\left(\mathcal{L}_{k}-\left\langle\mathcal{L}_{k}\right\rangle \mathcal{N}\right)$ is an operator annihilating the ferromagnetic vacuum.

Degrees of freedom. A deformation with the boost of $\mathcal{Q}_{2}$ only leads to a shift of the charge $\mathcal{Q}_{r}$ by the charge $\mathcal{Q}_{r+1} \sim\left[\mathcal{B}\left[\mathcal{Q}_{2}\right], \mathcal{Q}_{r}\right]$ (2.14). The corresponding degrees of freedom are accounted for by a different set of deformations (cf section 3.5) and are hence not included in (3.15).

Moreover, also the charges $\mathcal{Q}_{k}$ are defined only modulo identification of spectator legs (2.7). Consequently, due to the identifications (3.20), also $\mathcal{B}\left[\mathcal{Q}_{k}\right]$ is defined modulo some local operators. This ambiguity is not a problem though because all degrees of freedom resulting from deformations through local operators have already been taken into account in (3.8).

Example. As an example for the deformation (3.15), consider again the $\mathfrak{g l}(K)$ spin chain with the nearest neighbor Hamiltonian $\mathcal{Q}_{2}^{(0)}=[1]-[2,1]$. By virtue of $(2.14)$, the next higher commuting charge $\mathcal{Q}_{3}^{(0)}$ is given by

$$
\begin{aligned}
\mathcal{Q}_{3}^{(0)} & =-\frac{\mathrm{i}}{2}\left[\mathcal{B}\left[\mathcal{Q}_{2}^{(0)}, \mathcal{Q}_{2}^{(0)}\right]\right] \\
& =-\frac{\mathrm{i}}{2}[\mathcal{B}[[1]]-\mathcal{B}[[2,1]],[1]-[2,1]] \\
& =-\frac{\mathrm{i}}{2}([2,3,1]-[3,1,2])
\end{aligned}
$$

where for example $\mathcal{B}[[2,1]]$ is the boost of the permutation operator $[2,1]$. Now, deforming $\mathcal{Q}_{2}^{(0)}$ with $\mathcal{B}\left[\mathcal{Q}_{3}^{(0)}\right]$, yields

$$
\begin{aligned}
\mathcal{Q}_{2}^{(1)} & =\mathrm{i}\left[\mathcal{B}\left[\mathcal{Q}_{3}^{(0)}\right], \mathcal{Q}_{2}^{(0)}\right] \\
& =\frac{1}{2}[\mathcal{B}[[2,3,1]]-\mathcal{B}[[3,1,2]],[1]-[2,1]] \\
& =\frac{1}{2}(-2 \mathcal{B}[[1,3,2]]+2 \mathcal{B}[[2,1,3]]-[2,3,4,1]+[2,4,1,3]+[3,1,4,2]-[4,1,2,3]) \\
& =\frac{1}{2}(-2[1]+2[2,1]-[2,3,4,1]+[2,4,1,3]+[3,1,4,2]-[4,1,2,3]),
\end{aligned}
$$

where in the last line the two boost operators $\mathcal{B}[[1,3,2]]$ and $\mathcal{B}[[2,1,3]]$ were identified according to the prescription (3.20). Deforming $\mathcal{Q}_{3}^{(0)}$ in the same fashion results in

$$
\begin{aligned}
\mathcal{Q}_{3}^{(1)}=\mathrm{i}\left[\mathcal{B}\left[\mathcal{Q}_{3}^{(0)}\right], \mathcal{Q}_{3}^{(0)}\right] & \\
= & \frac{\mathrm{i}}{4}([2,4,3,1]+[3,2,4,1]-[4,1,3,2]-[4,2,1,3] \\
& \quad-2[2,3,4,5,1]+2[2,3,5,1,4]+2[2,4,1,5,3]-2[2,5,1,3,4] \\
& +2[3,1,4,5,2]-2[3,1,5,2,4]-2[4,1,2,5,3]+2[5,1,2,3,4]),
\end{aligned}
$$

and the deformed $\mathcal{Q}_{2}(\lambda)$ and $\mathcal{Q}_{3}(\lambda)$ indeed commute up to terms of order $\mathcal{O}\left(\lambda^{2}\right)$. Reinserting these expressions into the differential equation (3.15) and further expanding in the deformation parameter $\lambda$ recursively yields the higher order terms of the deformed charges $\mathcal{Q}_{2}(\lambda)$ and $\mathcal{Q}_{3}(\lambda)$. 


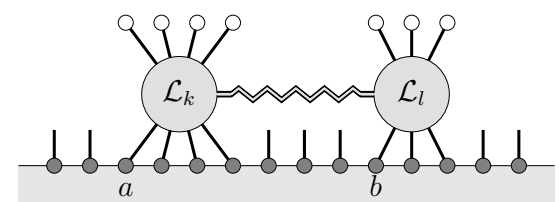

Figure 6. A bilocal operator $\left[\mathcal{L}_{k} \mid \mathcal{L}_{l}\right]$ can be constructed from two local operators by summing over all positions with $\mathcal{L}_{k}$ acting on the left of $\mathcal{L}_{l}, a \leqslant b$.

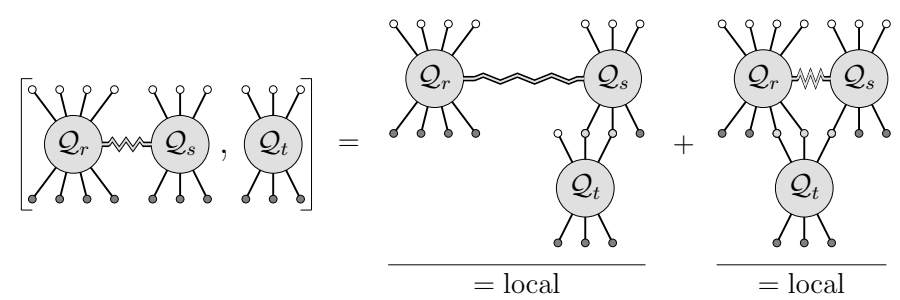

Figure 7. The commutator of a bilocal operator composed of two local charges $\mathcal{Q}_{r}$ and $\mathcal{Q}_{s}$ with a local charge $\mathcal{Q}_{t}$ gives a local result: when the two parts of the bilocal operator are well separated, the commutator either vanishes (if the two local charges commute with each other), or yields a bilocal operator in which one part consists of boundary terms only (if the two local charges commute up to boundary terms), which on an infinite chain amounts to a local operator. Also when both parts of the bilocal operator interact with the local charge at the same time, one gets a nonvanishing local contribution.

\subsection{Bilocal operators}

Further candidates for deformation generators are bilocal operators, which can be constructed from any two local operators, see figure $6^{2}$

$$
\left[\mathcal{L}_{j} \mid \mathcal{L}_{k}\right]:=\sum_{a, b} \Theta\left(\left(b+\frac{1}{2}\left|\mathcal{L}_{k}\right|\right)-\left(a+\frac{1}{2}\left|\mathcal{L}_{j}\right|\right)\right) \frac{1}{2}\left\{\mathcal{L}_{j}(a), \mathcal{L}_{k}(b)\right\}
$$

Here, $\left\{\mathcal{L}_{j}(a), \mathcal{L}_{k}(b)\right\}$ denotes the anticommutator of the local operators $\mathcal{L}_{j}(a)$ and $\mathcal{L}_{k}(b)$, which act starting at spin sites $a$ and $b$. The bilocal operator $\left[\mathcal{L}_{j} \mid \mathcal{L}_{k}\right]$ is constructed such that the sum of terms where $\mathcal{L}_{j}$ acts on either side of $\mathcal{L}_{k}$ equals the anticommutator

$$
\left[\mathcal{L}_{j} \mid \mathcal{L}_{k}\right]+\left[\mathcal{L}_{k} \mid \mathcal{L}_{j}\right]=\frac{1}{2}\left\{\mathcal{L}_{j}, \mathcal{L}_{k}\right\}
$$

Similar to the case of boost operators, commuting a bilocal with a local operator in general yields a combination of bilocal and local operators

$$
\left[\left[\mathcal{L}_{j} \mid \mathcal{L}_{k}\right], \mathcal{L}_{l}\right]=\left[\left[\mathcal{L}_{j} \mid \mathcal{L}_{l}\right], \mathcal{L}_{k}\right]+\left[\mathcal{L}_{j} \mid\left[\mathcal{L}_{k}, \mathcal{L}_{l}\right]\right]+\mathcal{L}_{m}
$$

However, if the underlying local operators commute, the commutator becomes local, cf figure 7. Hence, bilocal operators that are constructed from the commuting charges $\mathcal{Q}_{r}$ yield

2 We define the step function $\Theta(x)=0,1 / 2,1$ for $x<0, x=0, x>0$, respectively. 
admissible deformations ${ }^{3}$,

$$
\frac{\mathrm{d}}{\mathrm{d} \lambda} \mathcal{Q}_{t}(\lambda)=\mathrm{i}\left[\left[\mathcal{Q}_{r}(\lambda) \mid \mathcal{Q}_{s}(\lambda)\right], \mathcal{Q}_{t}(\lambda)\right], \quad s>r=2, \ldots, \infty,
$$

which result in deformed charges $\mathcal{Q}_{t}$ that are local. As for the boost operators, the deformation $\mathcal{X}(\lambda)=\left[\mathcal{Q}_{r}(\lambda) \mid \mathcal{Q}_{s}(\lambda)\right]$ directly depends on the charges $\mathcal{Q}_{r, s}$. Here the dependence is even quadratic and hence

$\mathcal{X}^{(0)}=\left[\mathcal{Q}_{r}^{(0)} \mid \mathcal{Q}_{s}^{(0)}\right], \quad \mathcal{X}^{(1)}=\left[\mathcal{Q}_{r}^{(1)} \mid \mathcal{Q}_{s}^{(0)}\right]+\left[\mathcal{Q}_{r}^{(0)} \mid \mathcal{Q}_{s}^{(1)}\right], \quad \mathcal{X}^{(2)}=\cdots$.

Bilocal operator identifications. As is the case for boost operators, bilocal operators are defined modulo local contributions. Again, this is due to the equivalence of local homogeneous operators that differ only by spectator legs (2.6), (2.7). More precisely, the following identifications can be made, where! again denotes a spectator leg ${ }^{4}$

$$
\begin{aligned}
& \left.\left[\mathcal{L}_{j}\right\rceil \mid \mathcal{L}_{k}\right]=\left[\mathcal{L}_{j} \mid \mathcal{L}_{k}\right]-\frac{1}{2} \sum_{a} \frac{1}{2}\left\{\mathcal{L}_{j}(a) \mathcal{L}_{k}\left(a-\left\lfloor\frac{1}{2}\left|\mathcal{L}_{k}\right|-\frac{1}{2}\left|\mathcal{L}_{j}\right|\right\rfloor\right)\right\}, \\
& {\left[\mathcal{L}_{j} \mid \mathcal{L}_{k} !\right]=\left[\mathcal{L}_{j} \mid \mathcal{L}_{k}\right]+\frac{1}{2} \sum_{b} \frac{1}{2}\left\{\mathcal{L}_{j}\left(b-\left\lfloor\frac{1}{2}\left|\mathcal{L}_{j}\right|-\frac{1}{2}\left|\mathcal{L}_{k}\right|\right\rfloor\right) \mathcal{L}_{k}(b)\right\}-\left\langle\mathcal{L}_{k}\right\rangle \mathcal{L}_{j},} \\
& {\left[\mathcal{L}_{j} \mid ! \mathcal{L}_{k}\right]=\left[\mathcal{L}_{j} \mid \mathcal{L}_{k}\right]-\frac{1}{2} \sum_{b} \frac{1}{2}\left\{\mathcal{L}_{j}\left(b-\left\lceil\frac{1}{2}\left|\mathcal{L}_{j}\right|-\frac{1}{2}\left|\mathcal{L}_{k}\right|\right\rceil\right) \mathcal{L}_{k}(b)\right\},} \\
& {\left[\left\lceil\mathcal{L}_{j} \mid \mathcal{L}_{k}\right]=\left[\mathcal{L}_{j} \mid \mathcal{L}_{k}\right]+\frac{1}{2} \sum_{a} \frac{1}{2}\left\{\mathcal{L}_{j}(a) \mathcal{L}_{k}\left(a-\left\lceil\frac{1}{2}\left|\mathcal{L}_{k}\right|-\frac{1}{2}\left|\mathcal{L}_{j}\right|\right]\right)\right\}-\left\langle\mathcal{L}_{j}\right\rangle \mathcal{L}_{k} .\right.}
\end{aligned}
$$

While the first correction terms on the right-hand side of the identifications follow straightforwardly from the definition (3.24), the addition/subtraction of $\mathcal{L}_{j, k}$ is necessary for a correct regularization: similar to the case of boost operators, for example, $\left[\mathcal{L}_{j} \mid \mathcal{L}_{k} !\right]$ and $\left[\mathcal{L}_{j} \mid \mathcal{L}_{k}\right]$ act differently at the right boundary of a finite chain. When the length of the chain is taken to infinity, the difference becomes the local operator $\left\langle\mathcal{L}_{k}\right\rangle \cdot \mathcal{L}_{j}$. Here, $\left\langle\mathcal{L}_{k}\right\rangle$ are the same constants as in (3.20).

Degrees of freedom. The identifications (3.29) show that two pairs of local operators that differ only by spectator legs yield the same bilocal operator, up to local terms,

$$
\mathcal{L}_{i} \simeq \mathcal{L}_{k}, \quad \mathcal{L}_{j} \simeq \mathcal{L}_{l} \text { (up to spectator legs) } \quad \Longrightarrow \quad\left[\mathcal{L}_{i} \mid \mathcal{L}_{j}\right]=\left[\mathcal{L}_{k} \mid \mathcal{L}_{l}\right]+\mathcal{L}_{r} .
$$

Due to this ambiguity, there remains some arbitrariness in the definition of bilocal operators (3.24): the 'overlap' between the two local operators for instance can be adjusted through the addition of local operators. As in the case of boost operators, this ambiguity is not troublesome, since all deformations by local homogeneous operators $\mathcal{L}_{r}$ can be absorbed into the similarity transformations (3.8). The definition (3.24) is chosen in favor of the identity (3.25), which immediately implies that

$$
\left[\left[\mathcal{Q}_{r} \mid \mathcal{Q}_{s}\right]+\left[\mathcal{Q}_{s} \mid \mathcal{Q}_{r}\right], \mathcal{Q}_{t}\right]=0
$$

Hence, $\left[\mathcal{Q}_{r} \mid \mathcal{Q}_{s}\right]$ and $\left[\mathcal{Q}_{s} \mid \mathcal{Q}_{r}\right]$ generate only one degree of freedom.

Note on boost operators. Observe that the boost operators (3.17) can formally be written as particular bilocal operators

$$
\mathcal{B}\left[\mathcal{L}_{k}\right]=\frac{1}{2}\left[\mathcal{N} \mid \mathcal{L}_{k}\right]-\frac{1}{2}\left[\mathcal{L}_{k} \mid \mathcal{N}\right],
$$

3 Note that the commutator of higher multilocal operators with local operators always yields multilocal operators again. Thus higher multilocal operators cannot be used to generate local structures.

4 The floor and ceiling functions are defined as $\lfloor x\rfloor:=\max \{z \in \mathbb{Z}: z \leqslant x\},\lceil x\rceil:=\min \{z \in \mathbb{Z}: z \geqslant x\}$. 
where $\mathcal{N}$ denotes the length operator as introduced in (3.20). The equivalence is due to the fact that in $\left[\mathcal{N} \mid \mathcal{L}_{k}\right]$ the operator $\mathcal{N}$ counts the spin sites to the left of $\mathcal{L}_{k} .{ }^{5}$ Correspondingly, the operator identifications in (3.20) and (3.29) are compatible. The identification would allow us to work with bilocal operators only and thus simplify the framework slightly.

Example. As an example for a deformation through bilocal operators, consider once more the $\mathfrak{g l}(K)$ spin chain. As presented in the previous example, the first two commuting nearest neighbor charges are

$$
\mathcal{Q}_{2}^{(0)}=[1]-[2,1], \quad \mathcal{Q}_{3}^{(0)}=-\frac{\mathrm{i}}{2}([2,3,1]-[3,1,2]) .
$$

Following (3.27), the first-order deformation of the charge $\mathcal{Q}_{2}$ is given by

$$
\begin{aligned}
\mathcal{Q}_{2}^{(1)}= & \mathrm{i}\left[\left[\mathcal{Q}_{2}^{(0)} \mid \mathcal{Q}_{3}^{(0)}\right], \mathcal{Q}_{2}^{(0)}\right] \\
= & \frac{1}{2}[[[1]-[2,1] \mid[2,3,1]-[3,1,2]],[1]-[2,1]] \\
= & \frac{1}{4}(-4[[1] \mid[1,3,2]]+4[[1] \mid[2,1,3]]+4[[2,1] \mid[1,3,2]] \\
& -4[[2,1] \mid[2,1,3]]+4[1]-2[2,3,1]-2[3,1,2]-2[2,1,4,3]-2[2,3,4,1] \\
& +2[2,4,1,3]+[2,4,3,1]+2[3,1,4,2]+[3,2,4,1]-2[3,4,1,2]-2[4,1,2,3] \\
& +[4,1,3,2]+[4,2,1,3]) \\
= & \frac{1}{4}(-4[1]+8[2,1]-2[2,3,1]-2[3,1,2]-2[2,1,4,3] \\
& -2[2,3,4,1]+2[2,4,1,3]+[2,4,3,1]+2[3,1,4,2]+[3,2,4,1]-2[3,4,1,2] \\
& -2[4,1,2,3]+[4,1,3,2]+[4,2,1,3]),
\end{aligned}
$$

where in the last line the bilocal operators were identified according to the rules (3.29). Deforming $\mathcal{Q}_{3}^{(0)}$ in the same fashion, the resulting charges $\mathcal{Q}_{2}$ and $\mathcal{Q}_{3}$ commute up to terms of order $\mathcal{O}\left(\lambda^{2}\right)$. The higher order terms of $\mathcal{Q}_{2}$ and $\mathcal{Q}_{3}$ can be obtained by successive reinsertion of the deformed charges into (3.27) and further expansion in $\lambda$.

\subsection{Basis of charges}

The operators presented above generate almost all admissible deformations of the form (3.1). However, taking linear combinations of the charges $\mathcal{Q}_{r}$ certainly does not change their algebra and therefore yields another type of allowed deformations

$$
\frac{\mathrm{d}}{\mathrm{d} \lambda} \mathcal{Q}_{r}(\lambda)=\mathcal{Q}_{s}(\lambda), \quad r, s=2, \ldots, \infty
$$

While the transformations (3.8) describe a change of basis within the space of local homogeneous operators, this type of deformation represents a change of basis within the algebra of charges $\mathcal{Q}_{r}$.

In order to analyze all admissible deformations in a common framework, we consider $\mathcal{Q}_{r}$ as the $r$ 'th component of a vector $\mathcal{Q}=\mathfrak{E}_{r} \mathcal{Q}_{r}$. A rotation generator $\mathfrak{G}_{r, s}$ acts on the basis vector $\mathfrak{E}_{k}$ as

$$
\left[\mathfrak{G}_{r, s}, \mathfrak{E}_{t}\right]=-\mathrm{i} \delta_{r, t} \mathfrak{E}_{s}
$$

\footnotetext{
5 For infinite chains this number is infinite. The infinity cancels in the antisymmetric definition in (3.32), but an unspecified finite shift remains as a regularization parameter. It is related to the freedom of shifting the origin (site 0 ) of the chain for the definition of boost operators, cf (3.12), (3.16).
} 
In other words, $\mathfrak{G}_{r, s}$ generate general linear transformations on the space of commuting charges. The deformation (3.35) can then be written as

$$
\frac{\mathrm{d}}{\mathrm{d} \lambda} \mathcal{Q}(\lambda)=\mathrm{i}\left[\mathfrak{G}_{r, s}, \mathcal{Q}(\lambda)\right], \quad r, s=2, \ldots, \infty .
$$

\subsection{Symmetry generators}

The charges $\mathcal{Q}(\lambda)$ of a symmetric spin chain should transform in a particular representation of the symmetry algebra. Assume that the generator $\mathcal{J}_{a}$ is represented on spin chain states by the operators $\mathcal{J}_{a}(\lambda)$. It is straightforward to deform the representation by means of the same differential equation as for the charges

$$
\frac{\mathrm{d}}{\mathrm{d} \lambda} \mathcal{J}_{a}(\lambda)=\mathrm{i}\left[\mathcal{X}(\lambda), \mathcal{J}_{a}(\lambda)\right] .
$$

Equation (3.3) guarantees that the structure constants of the commutation relations $\left[\mathcal{J}_{a}, \mathcal{J}_{b}\right]$ and $\left[\mathcal{J}_{a}, \mathcal{Q}\right]$ are preserved under the deformation. Since (3.38) generates similarity transformations, $\mathcal{J}_{a}(\lambda)$ is a one-parameter family of equivalent representations of a single undeformed algebra.

Let us first consider the symmetry to be a Lie algebra $\mathfrak{g}$ with commutation relations

$$
\left[\mathcal{J}_{a}, \mathcal{J}_{b}\right]=F_{a, b}^{c} \mathcal{J}_{c},
$$

and invariant charges

$$
\left[\mathcal{J}_{a}, \mathcal{Q}\right]=0 .
$$

Obviously these commutation relations are preserved by the deformation with undeformed structure constants $F_{a, b}^{c}$. This work focuses on deformations for which (3.38) is trivial: the deformations discussed above are invariant under the symmetry, $\left[\mathcal{X}_{k}(\lambda), \mathcal{J}_{a}(\lambda)\right]=0$, which leads to $\mathcal{J}_{a}(\lambda)=\mathcal{J}_{a}(0)$.

For integrable spin chains the Lie algebra furthermore extends to Yangian symmetry. Yangian symmetry implies an additional set of generators $\mathcal{Y}_{a}$ which transform in the adjoint representation of the Lie algebra $\mathfrak{g}$,

$$
\left[\mathcal{J}_{a}, \mathcal{Y}_{b}\right]=F_{a, b}^{c} \mathcal{Y}_{c},
$$

and which leave the conserved charges invariant

$$
\left[\mathcal{Y}_{a}, \mathcal{Q}\right]=0 \text {. }
$$

Additionally, the Yangian generators and the Lie algebra generators $\mathcal{J}_{a}$ have to satisfy the Serre relations [27]. These are given by

$$
\left[\mathcal{Y}_{a},\left[\mathcal{J}_{b}, \mathcal{Y}_{c}\right]\right]+\left[\mathcal{Y}_{b},\left[\mathcal{J}_{c}, \mathcal{Y}_{a}\right]\right]+\left[\mathcal{Y}_{c},\left[\mathcal{J}_{a}, \mathcal{Y}_{b}\right]\right]=\frac{1}{6} A_{a b c}^{\operatorname{def}}\left\{\mathcal{J}_{d}, \mathcal{J}_{e}, \mathcal{J}_{f}\right\}
$$

where $\{\ldots\}$ represents the sum over all six permutations of the enclosed generators and the coefficients $A_{a b c}^{\mathrm{def}}$ are given in terms of the structure constants and the Cartan matrix $C^{a, b}$

$$
A_{a b c}^{\mathrm{def}}=\frac{1}{4} F_{a, g}^{d} F_{b, h}^{e} F_{c, j}^{f} F_{d, e}^{j} C^{g, d} C^{h, e} .
$$

Since the deformation (3.1) in general preserves the algebra between the deformed quantities, the invariance property (3.42) is preserved if the Yangian generators are deformed in the same way as the charges $(4.3)$

$$
\frac{\mathrm{d}}{\mathrm{d} \lambda} \mathcal{Y}_{a}(\lambda)=\mathrm{i}\left[\mathcal{X}(\lambda), \mathcal{Y}_{a}(\lambda)\right]
$$


Because the change of basis deformation (3.37) only mixes the charges $\mathcal{Q}_{r}$ among themselves, it does not affect (3.42). Since the Lie algebra generators $\mathcal{J}_{a}$ are deformed (3.38) in the same way as the Yangian generators (3.45), also the Serre relations are preserved by the deformation.

The fact that the generating equation (3.1) preserves any algebra among the deformed operators $\mathcal{Q}$ might be particularly interesting for extending our deformation method to models in which also the Lie algebra representation $\mathcal{J}_{a}(\lambda)$ is deformed non-trivially. One such case was recently studied by Zwiebel [30], who found a differential equation reminiscent of ours.

\section{Geometry of the moduli space}

In the previous section we have found several admissible deformations $\mathcal{X}_{k}$ which generate long-range integrable spin chains of infinite extent by means of a simple differential equation. These are deformations by local operators $\mathcal{L}_{l}$, boosted charges $\mathcal{B}\left[\mathcal{Q}_{k}\right]$, bilocal charges $\left[\mathcal{Q}_{r} \mid \mathcal{Q}_{s}\right]$ as well as changes $\mathfrak{G}_{m, n}$ of the basis of local operators. Taking all these operators into account results in multi-parameter deformations. In this section we shall discuss the dependence of the charges $\mathcal{Q}_{r}$ on the moduli. Qualitatively it will depend crucially on whether the differential equation obeys a flatness condition.

\subsection{Multi-parameter deformations}

There is nothing that prevents us from combining the various deformations into a system with multiple moduli $\left\{\xi_{j}\right\}=\left\{\alpha_{k}, \beta_{r, s}, \gamma_{m, n}, \varepsilon_{l}\right\}$. This is done by choosing the deformation $\mathcal{X}$ to be some linear combination ${ }^{6}$ of the $\left\{\mathcal{X}_{j}\right\}=\left\{\mathcal{B}\left[\mathcal{Q}_{k}\right],\left[\mathcal{Q}_{r} \mid \mathcal{Q}_{s}\right], \mathfrak{G}_{m, n}, \mathcal{L}_{l}\right\}$ (we sum over repeated indices)

$$
\mathcal{X}(\lambda)=\frac{\mathrm{d} \xi_{j}(\lambda)}{\mathrm{d} \lambda} \mathcal{X}_{j}(\lambda)
$$

We shall investigate how the charges $\mathcal{Q}_{r}$ depend on the moduli. Substituting the linear combination of deformation operators (4.1) into the generating equation (3.1) yields the differential equation

$$
\mathrm{d} \mathcal{Q}(\lambda)=\mathrm{i}\left[\mathcal{X}_{j}(\lambda), \mathcal{Q}(\lambda)\right] \mathrm{d} \xi_{j}(\lambda)
$$

For later purposes it will be very convenient to use the language of differential forms $\Xi=\mathcal{X}_{j} d \xi_{j}$ on moduli space. Including all operators discussed in section 3 , we find the most general generating equation

$\mathrm{d} \mathcal{Q}=\mathrm{i}\left[\mathcal{B}\left[\mathcal{Q}_{k}\right], \mathcal{Q}\right] \Pi_{k}+\mathrm{i}\left[\left[\mathcal{Q}_{r} \mid \mathcal{Q}_{s}\right], \mathcal{Q}\right] \Upsilon_{r, s}+\mathrm{i}\left[\mathfrak{G}_{m, n}, \mathcal{Q}\right] \Gamma_{m, n}+\mathrm{i}\left[\mathcal{L}_{l}, \mathcal{Q}\right] \Lambda_{l}$.

The coefficients $\Pi_{k}, \Upsilon_{r, s}, \Gamma_{m, n}, \Lambda_{l}$ are 1-forms on moduli space which parametrize the desired deformation. They can depend arbitrarily on any of the moduli $\xi_{j}$.

Note that, as before, the generating equation defines merely a one-parameter family of charges $\mathcal{Q}_{r}(\lambda)$. However, now we have the additional freedom to specify the functions $\xi_{j}\left(\lambda^{\prime}\right)$ which define a curve on moduli space. This means that the charges $\mathcal{Q}_{r}$ depend not only on a point $\xi_{j}(\lambda)$ in moduli space, but also on the shape of the curve $\xi_{j}\left(\lambda^{\prime}\right)$ connecting the undeformed model at $\lambda^{\prime}=0$ to the deformed model at $\lambda^{\prime}=\lambda$. In general one cannot expect the dependence on the shape of the curve to be trivial.

A further complication is that the deformation operators $\mathcal{X}_{j}\left(\lambda^{\prime}\right)$ are neither constants nor proper functions of the moduli $\xi_{j}$ : For the boost and bilocal deformations, $\mathcal{X}=$ $\mathcal{B}\left[\mathcal{Q}_{k}\right],\left[\mathcal{Q}_{r} \mid \mathcal{Q}_{s}\right]$, they actually depend on the solution $\mathcal{Q}_{r}\left(\lambda^{\prime}\right)$ of the differential equation

6 The linear combination may have $\lambda$-dependent coefficients. 
itself. This unusual feature complicates the treatment, but we can at least make use of a weaker fact: the differential of the deformations $\mathrm{d} \mathcal{X}_{j}\left(\lambda^{\prime}\right)$ (which is what is needed in practice) can be expressed through the differential of the charges $\mathrm{d} \mathcal{Q}_{k}\left(\lambda^{\prime}\right)$ which in turn is determined through the differential equation (4.2).

One can find a perturbative solution for the generating equation (4.2) with multiple parameters. The deformation curve $\xi_{j}\left(\lambda^{\prime}\right)$ is assumed to start at the origin $\xi_{j}(0)=0$ of the moduli space with the undeformed charges $\mathcal{Q}_{r}^{(0)}$. It is furthermore assumed to be confined to a small neighborhood of the origin. Then one can expand the solution $\mathcal{Q}(\lambda)$ in terms of small $\xi_{j}(\lambda)$ as follows:

$$
\begin{aligned}
\mathcal{Q}(\lambda)=\mathcal{Q}^{(0)}+ & \xi_{k}(\lambda) \mathrm{i}\left[\mathcal{X}_{k}^{(0)}, \mathcal{Q}^{(0)}\right]+\int_{0}^{\lambda} \mathrm{d} \xi_{k}\left(\lambda^{\prime}\right) \mathrm{i}\left[\mathcal{X}_{k}^{(1)}, \mathcal{Q}^{(0)}\right] \\
& +\int_{0}^{\lambda} \mathrm{d} \xi_{k}\left(\lambda^{\prime}\right) \xi_{l}\left(\lambda^{\prime}\right) \mathrm{i}\left[\mathcal{X}_{k}^{(0)}, \mathrm{i}\left[\mathcal{X}_{l}^{(0)}, \mathcal{Q}^{(0)}\right]\right]+\mathcal{O}\left(\xi^{3}\right) .
\end{aligned}
$$

Here we have expanded the deformation $\mathcal{X}_{k}(\lambda)=\mathcal{X}_{k}^{(0)}+\mathcal{X}_{k}^{(1)}+\cdots$ in powers of $\xi$. As explained above, we actually know the partial derivatives of $\mathcal{X}_{k}$ in all directions of the moduli space. We can therefore write $\mathcal{X}_{k}^{(1)}=\xi_{l}(\lambda) \mathcal{X}_{k}^{[l]}$. Splitting the integrals into their symmetric and antisymmetric part allows us to rewrite the expansion in a more illuminating fashion

$$
\begin{aligned}
\mathcal{Q}(\lambda)=\mathcal{Q}^{(0)}+ & \xi_{k}(\lambda) \mathrm{i}\left[\mathcal{X}_{k}^{(0)}, \mathcal{Q}^{(0)}\right]+\frac{1}{2} \xi_{k}(\lambda) \xi_{l}(\lambda)\left(\mathrm{i}\left[\mathcal{X}_{k}^{[l]}, \mathcal{Q}^{(0)}\right]+\mathrm{i}\left[\mathcal{X}_{k}^{(0)}, \mathrm{i}\left[\mathcal{X}_{l}^{(0)}, \mathcal{Q}^{(0)}\right]\right]\right) \\
& +\frac{1}{2} \int_{0}^{\lambda} \mathrm{d} \xi_{k}\left(\lambda^{\prime}\right) \xi_{l}\left(\lambda^{\prime}\right) \mathrm{i}\left[\mathcal{X}_{k}^{[l]}-\mathcal{X}_{l}^{[k]}+\mathrm{i}\left[\mathcal{X}_{k}^{(0)}, \mathcal{X}_{l}^{(0)}\right], \mathcal{Q}^{(0)}\right]+\mathcal{O}\left(\xi^{3}\right)
\end{aligned}
$$

\subsection{Connection and curvature}

The generating equation (4.2) can be interpreted as a parallel transport equation for the vector of commuting charges $\mathcal{Q}$

$$
\mathcal{D}_{\mathrm{ad}} \mathcal{Q}=0 \text {. }
$$

Here $\mathcal{D}_{\text {ad }}$ is the covariant derivative $\mathcal{D}$ in the adjoint representation

$$
\mathcal{D}:=d-\mathrm{i} \Xi, \quad \mathcal{D}_{\mathrm{ad}}:=d-\mathrm{i} \operatorname{ad}(\Xi), \quad \operatorname{ad}(\Xi) \mathcal{Q}:=[\Xi, \mathcal{Q}] .
$$

The operator-valued connection $\Xi$ which includes all the admissible deformations reads

$$
\Xi:=\mathcal{X}_{j} \mathrm{~d} \xi_{j}=\mathcal{B}\left[\mathcal{Q}_{k}\right] \Pi_{k}+\left[\mathcal{Q}_{r} \mid \mathcal{Q}_{s}\right] \Upsilon_{r, s}+\mathfrak{G}_{m, n} \Gamma_{m, n}+\mathcal{L}_{l} \Lambda_{l}
$$

This connection may or may not be flat: flatness would imply that the deformed charges $\mathcal{Q}(\lambda)$ are independent of the shape of the path $\xi\left(\lambda^{\prime}\right)$ along which they are parallel transported. They would only depend on the endpoint $\xi(\lambda)$ of the path and thus they could be defined as proper functions $\mathcal{Q}(\xi)$ on moduli space. In the expansion (4.5) one can observe the influence of flatness: the terms on the first line exclusively depend on the endpoint $\xi(\lambda)$ while the term on the second line requires some integrals over $\xi\left(\lambda^{\prime}\right)$. Importantly, the latter term is proportional to the curvature of the connection $\Xi$ and consequently it vanishes for a flat connection.

Let us now calculate the curvature of the connection $\Xi$. According to (4.7) it reads

$$
\mathrm{i} \mathcal{D}^{2}=\mathrm{d} \Xi-\mathrm{i} \Xi \wedge \Xi \text {. }
$$

In the following we shall neglect the deformations $\Lambda_{l}$ by local operators. This is favorable for several reasons: First, these deformations turn out to form an ideal, they do not influence the curvature components associated with boosts, bilocal and basis change transformations. Second, it allows us to discard local contributions originating from the other deformations at 
most steps of the calculation. These would be hard to treat quantitatively and in full generality. And last but not least the deformations by local operators are irrelevant in the sense that they have no impact on the spectrum of finite chains. We are thus left with a connection

$$
\Xi=\Pi+\Upsilon+\Gamma
$$

The operator-valued 1-forms $\Pi, \Upsilon$ and $\Gamma$ are given by

$$
\Pi:=\mathcal{P}_{k} \Pi_{k}, \quad \Upsilon:=\mathcal{Y}_{r, s} \Upsilon_{r, s}, \quad \Gamma:=\mathfrak{G}_{m, n} \Gamma_{m, n} .
$$

with the abbreviations $\mathcal{P}_{k}, \mathcal{Y}_{r, s}$ for the boost and bilocal charges

$$
\mathcal{P}_{k}:=\mathcal{B}\left[\mathcal{Q}_{k}\right], \quad \mathcal{Y}_{r, s}:=\left[\mathcal{Q}_{r} \mid \mathcal{Q}_{s}\right] .
$$

The curvature is now given by

$$
\mathrm{i} \mathcal{D}^{2}=\mathrm{d} \Pi+\mathrm{d} \Upsilon+\mathrm{d} \Gamma-\mathrm{i}(\Pi+\Upsilon+\Gamma) \wedge(\Pi+\Upsilon+\Gamma) .
$$

First, consider the bilocal connection $\Upsilon$. Given that $\mathcal{D}_{\text {ad }} \mathcal{Q}=0$, a short calculation shows that its exterior derivative is given by

$$
\begin{aligned}
\mathrm{d} \Upsilon= & \left(\left[\mathrm{d} \mathcal{Q}_{r} \mid \mathcal{Q}_{s}\right]+\left[\mathcal{Q}_{r} \mid \mathrm{d} \mathcal{Q}_{s}\right]\right) \wedge \Upsilon_{r, s}+\mathcal{Y}_{r, s} \mathrm{~d} \Upsilon_{r, s} \\
= & \mathrm{i}\left(\left[\left[\mathcal{Y}_{t, u}, \mathcal{Q}_{r}\right] \mid \mathcal{Q}_{s}\right]+\left[\mathcal{Q}_{r} \mid\left[\mathcal{Y}_{t, u}, \mathcal{Q}_{s}\right]\right]\right) \Upsilon_{t, u} \wedge \Upsilon_{r, s} \\
& +\mathrm{i}\left(\left[\left[\mathcal{P}_{k}, \mathcal{Q}_{r}\right] \mid \mathcal{Q}_{s}\right]+\left[\mathcal{Q}_{r} \mid\left[\mathcal{P}_{k}, \mathcal{Q}_{s}\right]\right]\right) \Pi_{k} \wedge \Upsilon_{r, s} \\
& +\left(\delta_{m, r} \mathcal{Y}_{n, s}+\delta_{m, s} \mathcal{Y}_{r, n}\right) \Gamma_{m, n} \wedge \Upsilon_{r, s}+\mathcal{Y}_{r, s} \mathrm{~d} \Upsilon_{r, s},
\end{aligned}
$$

where it was used that $\left[\mathfrak{G}_{m, n}, \mathcal{Q}\right]_{r}=-\mathrm{i} \delta_{m, r} \mathcal{Q}_{n}$. Similarly, the exterior derivative of the boost connection $\Pi$ reads

$$
\begin{aligned}
\mathrm{d} \Pi= & \mathcal{B}\left[d \mathcal{Q}_{k}\right] \wedge \Pi_{k}+\mathcal{P}_{k} \mathrm{~d} \Pi_{k} \\
= & \mathrm{i} \mathcal{B}\left[\left[\mathcal{P}_{l}, \mathcal{Q}_{k}\right]\right] \Pi_{l} \wedge \Pi_{k}+\mathrm{i} \mathcal{B}\left[\left[\mathcal{Y}_{r, s}, \mathcal{Q}_{k}\right]\right] \Upsilon_{r, s} \wedge \Pi_{k} \\
& +\delta_{m, k} \mathcal{P}_{n} \Gamma_{m, n} \wedge \Pi_{k}+\mathcal{P}_{k} \mathrm{~d} \Pi_{k} .
\end{aligned}
$$

Because the operators $\mathfrak{G}_{m, n}$ that generate basis changes do not depend on the coordinates $\xi_{j}$, the exterior derivative of the connection $\Gamma$ simply reads

$$
\mathrm{d} \Gamma=\mathfrak{G}_{m, n} \mathrm{~d} \Gamma_{m, n} .
$$

Using the Jacobi identity and (3.31), one finds

$$
\begin{aligned}
i \Upsilon \wedge \Upsilon & =\frac{\mathrm{i}}{2}\left[\left[\mathcal{Q}_{r} \mid \mathcal{Q}_{s}\right],\left[\mathcal{Q}_{t} \mid \mathcal{Q}_{u}\right]\right] \Upsilon_{r, s} \wedge \Upsilon_{t, u} \\
& =-\mathrm{i}\left(\left[\left[\mathcal{Y}_{t, u}, \mathcal{Q}_{r}\right] \mid \mathcal{Q}_{s}\right]+\left[\mathcal{Q}_{r} \mid\left[\mathcal{Y}_{t, u}, \mathcal{Q}_{s}\right]\right]\right) \Upsilon_{r, s} \wedge \Upsilon_{t, u},
\end{aligned}
$$

where it was further used that the commutator of two bilocal operators evaluates to (cf figure 8)

$$
\begin{array}{r}
{\left[\left[\mathcal{Q}_{r} \mid \mathcal{Q}_{s}\right],\left[\mathcal{Q}_{t} \mid \mathcal{Q}_{u}\right]\right]=\left[\left[\mathcal{Q}_{r},\left[\mathcal{Q}_{t} \mid \mathcal{Q}_{u}\right]\right] \mid \mathcal{Q}_{s}\right]+\left[\mathcal{Q}_{r} \mid\left[\mathcal{Q}_{s},\left[\mathcal{Q}_{t} \mid \mathcal{Q}_{u}\right]\right]\right]} \\
+\left[\left[\left[\mathcal{Q}_{r} \mid \mathcal{Q}_{s}\right], \mathcal{Q}_{t}\right] \mid \mathcal{Q}_{u}\right]+\left[\mathcal{Q}_{t} \mid\left[\left[\mathcal{Q}_{r} \mid \mathcal{Q}_{s}\right], \mathcal{Q}_{u}\right]\right]+\text { local }
\end{array}
$$

Similarly, one finds

$$
\begin{aligned}
& \mathrm{i} \Pi \wedge \Pi=\frac{\mathrm{i}}{2}\left[\mathcal{B}\left[\mathcal{Q}_{k}\right], \mathcal{B}\left[\mathcal{Q}_{l}\right]\right] \Pi_{k} \wedge \Pi_{l}=-\mathrm{i} \mathcal{B}\left[\left[\mathcal{P}_{l}, \mathcal{Q}_{k}\right]\right] \Pi_{k} \wedge \Pi_{l} \\
& \begin{aligned}
\mathrm{i} \Pi \wedge \Upsilon+\mathrm{i} \Upsilon \wedge \Pi & =-\frac{\mathrm{i}}{2}\left[\mathcal{P}_{k}, \mathcal{Y}_{r, s}\right] \Pi_{k} \wedge \Upsilon_{r, s}-\frac{\mathrm{i}}{2}\left[\mathcal{Y}_{r, s}, \mathcal{P}_{k}\right] \Upsilon_{r, s} \wedge \Pi_{k} \\
& =\left(-\mathrm{i} \mathcal{B}\left[\left[\mathcal{Y}_{r, s}, \mathcal{Q}_{k}\right]\right]+\mathrm{i}\left[\mathcal{Q}_{r} \mid\left[\mathcal{P}_{k}, \mathcal{Q}_{s}\right]\right]+\mathrm{i}\left[\left[\mathcal{P}_{k}, \mathcal{Q}_{r}\right] \mid \mathcal{Q}_{s}\right]\right) \Pi_{k} \wedge \Upsilon_{r, s}
\end{aligned}
\end{aligned}
$$

Since the generators $\mathfrak{G}_{m, n}$ commute with the boost and bilocal operators $\mathcal{P}_{k}$ and $\mathcal{Y}_{r, s}$, the remaining terms of the curvature (4.13) are 

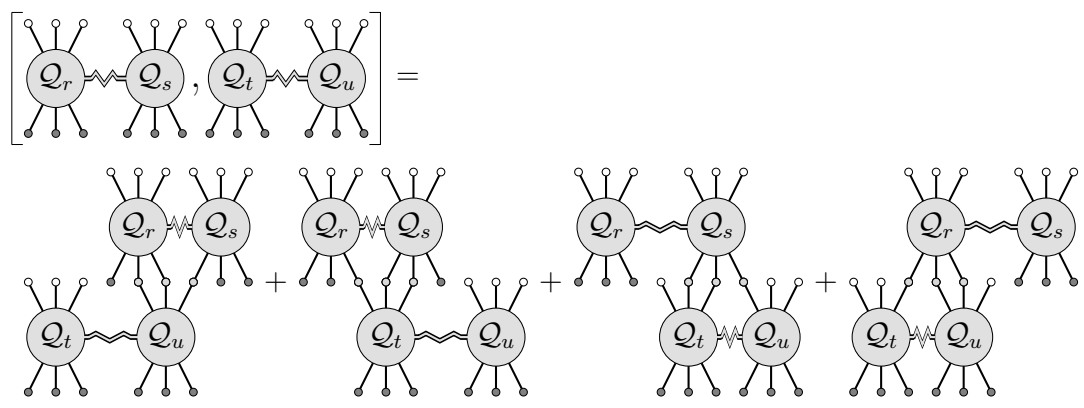

Figure 8. Graphical representation of the commutator (4.18) of two bilocal operators. Since the local charges commute with each other, the only contributing terms (up to local operators) are those where both local parts of one bilocal operator overlap with one of the local charges of the other bilocal operator.

$$
\begin{aligned}
& \mathrm{i} \Gamma \wedge \Gamma=\frac{\mathrm{i}}{2}\left[\mathfrak{G}_{m, n}, \mathfrak{G}_{p, q}\right] \Gamma_{m, n} \wedge \Gamma_{p, q}=\delta_{n, p} \mathfrak{G}_{m, q} \Gamma_{m, n} \wedge \Gamma_{p, q}, \\
& \mathrm{i} \Pi \wedge \Gamma+\mathrm{i} \Gamma \wedge \Pi=-\mathrm{i}\left[\mathcal{P}_{k}, \mathfrak{G}_{m, n}\right] \Pi_{k} \wedge \Gamma_{m, n}=0, \\
& \mathrm{i} \Upsilon \wedge \Gamma+\mathrm{i} \Gamma \wedge \Upsilon=-\mathrm{i}\left[\mathcal{Y}_{r, s}, \mathfrak{G}_{m, n}\right] \Upsilon_{r, s} \wedge \Gamma_{m, n}=0 .
\end{aligned}
$$

Hence, the curvature reduces to

$$
\begin{aligned}
\mathrm{i} \mathcal{D}^{2}=\mathcal{P}_{k}\left(\mathrm{~d} \Pi_{k}+\Gamma_{p, k} \wedge \Pi_{p}\right)+\mathcal{Y}_{r, s}\left(\mathrm{~d} \Upsilon_{r, s}+\Gamma_{p, r} \wedge \Upsilon_{p, s}+\Gamma_{p, s} \wedge \Upsilon_{r, p}\right) \\
+\mathfrak{G}_{m, n}\left(\mathrm{~d} \Gamma_{m, n}-\Gamma_{m, p} \wedge \Gamma_{p, n}\right) .
\end{aligned}
$$

It is curious to see that the nonlinear components of the curvature are all due to basis change deformations $\Gamma_{m, n}$. The group structure underlying the connection consists of a general linear group $\left(\Gamma_{m, n}\right)$ and two abelian ideals $\left(\Pi_{k}, \Upsilon_{r, s}\right)$.

\subsection{Flatness}

According to (4.22) the covariant derivative defined in (4.7) is flat if

$$
\begin{aligned}
& 0=\mathrm{d} \Pi_{k}+\Gamma_{p, k} \wedge \Pi_{p}, \\
& 0=\mathrm{d} \Upsilon_{r, s}+\Gamma_{p, r} \wedge \Upsilon_{p, s}+\Gamma_{p, s} \wedge \Upsilon_{r, p}, \\
& 0=\mathrm{d} \Gamma_{m, n}-\Gamma_{m, p} \wedge \Gamma_{p, n} .
\end{aligned}
$$

Flatness implies that the charges $\mathcal{Q}_{r}(\lambda)$ depend only on the final position $\xi(\lambda)$ in moduli space; they are independent of the shape of the path $\xi\left(\lambda^{\prime}\right)$. In other words, the charges are singlevalued functions $\mathcal{Q}_{r}(\xi)$ on moduli space. An expansion of the charges in the deformation parameters therefore exists, see figure 9 ,

$$
\mathcal{Q}(\xi)=\mathcal{Q}^{(0)}+\xi_{j} \mathcal{Q}^{[j]}+\frac{1}{2} \xi_{j} \xi_{k} \mathcal{Q}^{[j, k]}+\mathcal{O}\left(\xi^{3}\right)
$$

According to (4.5) the first two expansion coefficients read

$$
\begin{aligned}
& \mathcal{Q}^{[k]}=\mathrm{i}\left[\mathcal{X}_{k}^{(0)}, \mathcal{Q}^{(0)}\right], \\
& \begin{aligned}
\mathcal{Q}^{[k, l]} & =\mathrm{i}\left[\mathcal{X}_{l}^{[k]}, \mathcal{Q}^{(0)}\right]+\mathrm{i}\left[\mathcal{X}_{l}^{(0)}, \mathcal{Q}^{[k]}\right] . \\
& =\mathrm{i}\left[\mathcal{X}_{k}^{[l]}, \mathcal{Q}^{(0)}\right]+\mathrm{i}\left[\mathcal{X}_{k}^{(0)}, \mathcal{Q}^{[l]}\right]
\end{aligned}
\end{aligned}
$$

Note that if the deformation (4.3) does not incorporate a change of basis, i.e. $\Gamma \equiv 0$, exact boost and bilocal connections $\Pi_{k}=\mathrm{d} \alpha_{k}, \Upsilon_{r, s}=\mathrm{d} \beta_{r, s}$ lead to a flat connection and expansion 


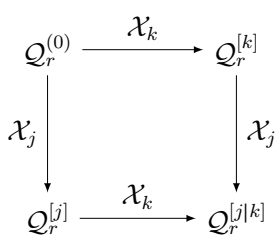

Figure 9. The deformed charges are independent of the shape of the path within the moduli space only if the connection is flat. In this case, first deforming in the direction $\xi_{l}$ and then in the direction $\xi_{k}$ yields the same result as first deforming in the direction $\xi_{k}$ and then in the direction $\xi_{l}$. Hence, the charges can be directly expanded (4.26) in the deformation parameters $\xi_{j}$.

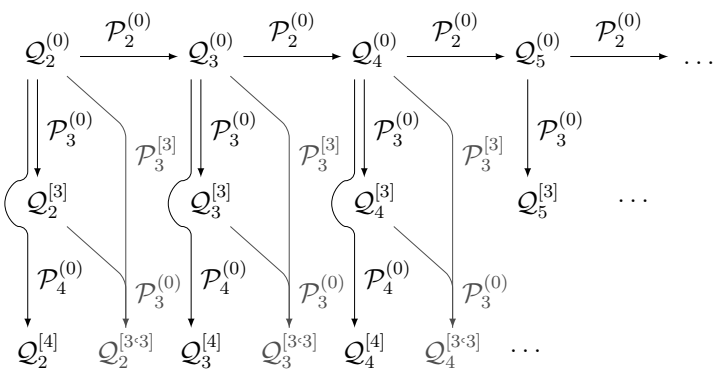

Figure 10. The boost deformations commute among themselves. Thus if for instance $\Gamma \equiv \Upsilon \equiv 0$ and $\Pi_{k}=\mathrm{d} \alpha_{k}$, the boost-deformed charges can be directly expanded in the deformation parameters $\alpha_{k}, \operatorname{cf}$ (2.14). The zeroth-order higher charges are generated by the boost $\mathcal{P}_{2}^{(0)}$ while the coefficient $\mathcal{Q}_{r}^{[k]}$ of $\alpha_{k}$ is given by the commutator of $\mathcal{P}_{k}^{(0)}$ with the corresponding charge $\mathcal{Q}_{r}^{(0)}$. Note in particular that coefficients of higher powers of $\alpha_{k}$ are generated by the higher order terms of the charge and boost operators drawn in gray, e.g. $\mathcal{Q}_{r}^{[3,3]} \sim\left[\mathcal{P}_{3}^{[3]}, \mathcal{Q}_{r}^{(0)}\right]+\left[\mathcal{P}_{3}^{(0)}, \mathcal{Q}_{r}^{[3]}\right]$.

in terms of $\alpha_{k}$ and $\beta_{r, s}$, cf figure 10. On the other hand, when $\Gamma$ is chosen to be nonzero but such that it satisfies (4.25), the boost and bilocal connections $\Pi, \Upsilon$ must be modified in order to keep the connection flat, i.e. in order to satisfy (4.23), (4.24). In the following section, explicit forms $\Pi, \Upsilon$ in terms of moduli $\alpha_{k}, \beta_{r, s}$ will be constructed for a specific, nonzero choice of $\Gamma$. It will turn out that these forms yield a flat connection $\Pi+\Upsilon+\Gamma$.

\section{Interaction range}

In this section we will investigate the change of the interaction range of the integrable charges due to the various deformations. We present a parametrization that leads to a definite, minimal increase of the range by each deformation. A minimal range is necessary to make a comparison to earlier studies [24] where this feature is manifest. As previously the interaction range of a local operator $\mathcal{L}_{k}$ will be denoted by $\left|\mathcal{L}_{k}\right|$.

\subsection{First comparison to $\mathfrak{g l}(K)$ chains}

The set of deformation moduli discussed in section 3 qualitatively agrees with the set of moduli $\left\{\alpha_{k}, \beta_{r, s}, \gamma_{m, n}, \varepsilon_{l}\right\}$ of long-range integrable $\mathfrak{g l}(K)$ spin-chains proposed in [24] $]^{7}$. This

7 Also the deformation of the Yangian generators agrees qualitatively with the results of [25]: The bi-local terms remain undeformed while there are local deformations of $\mathcal{Y}_{a}$. The Serre relations defining the Yangian algebra are unmodified. 
is a useful indication that both constructions describe the same system and that we have not missed any admissible deformations. Nevertheless, the precise form of the deformations, cf (3.22), (3.34), does not match with those obtained in [24], there are two main differences:

The qualitative comparison suggests that the deformation of the lowest boost $\mathcal{P}_{3}$ should correspond to the lowest rapidity parameter $\alpha_{0}$ in [24]. Nevertheless the leading-order deformation (3.22) does not appear among the deformations in [24]. In particular, each deformation by $\mathcal{P}_{3}$ increases the range by two units such that $\left|\mathcal{Q}_{2}^{(1)}\right|=4$ in (3.22) as opposed to [24] where each power of $\alpha_{0}$ increases the range by merely one unit. Nevertheless the explorative study in [24] is complete up to range 6, and therefore our deformation by $\mathcal{P}_{3}$ must be among the deformations in [24]. The resolution to the puzzle is that $\alpha_{0}$ deformation in [24] is a combination of the boost deformations $\mathcal{P}_{3}$ and the change of basis deformation $\mathfrak{G}_{2,4}$. The combination must be chosen such that the range decreases by one step, which will be the topic of section 5.2. Furthermore higher order deformations should have a consistent pattern of ranges, see sections 5.3, 5.4. In section 5.5 it will be shown that this actually leads to a flat connection as discussed in the previous section.

The leading-order bilocal deformation (3.34) indeed agrees literally with the structure multiplying the coefficient $\gamma_{2,2} \beta_{2,3}$ in [24, 25]. The coefficient $\gamma_{2,2}$ appears because in [24] the final charges $\mathcal{Q}_{r}$ are given as linear combinations $\gamma_{r, s} \overline{\mathcal{Q}}_{s}$ of normalized charges $\overline{\mathcal{Q}}_{s}$. The latter contain only the deformation moduli $\alpha_{k}, \beta_{r, s}, \varepsilon_{l}$. Conversely, in our differential equation the change of basis deformations act at all points of the deformation path and thus mix with the other moduli. We should therefore aim to reproduce merely the normalized charges $\overline{\mathcal{Q}}_{s}$ of [24] using the moduli $\alpha_{k}, \beta_{r, s}, \varepsilon_{l}$ and at the end apply a finite change of basis $\mathcal{Q}_{r} \rightarrow \gamma_{r, s} \mathcal{Q}_{s}$.

Finally, as in the previous section, we will disregard deformations by local operators $\mathcal{L}_{l}$ and the corresponding moduli $\varepsilon_{l}$. As mentioned earlier and as to be explained in section 6 , they do not affect the quantities we are interested in and can be reintroduced easily through the similarity transformation (3.9).

\subsection{Boost connection at leading order}

First, consider the boost connection alone,

$$
\Pi=\mathcal{P}_{k} \Pi_{k}=\mathcal{B}\left[\mathcal{Q}_{k}\right] \Pi_{k} .
$$

As one would expect, the first-order deformation terms $\mathrm{i}\left[\mathcal{P}_{k}^{(0)}, \mathcal{Q}_{r}^{(0)}\right]$ generically have range $\left|\mathcal{Q}_{k}^{(0)}\right|+\left|\mathcal{Q}_{r}^{(0)}\right|-1$. In our examples, however, we observe that the longest contributions of some boosted charge precisely match with the longest contributions of another, undeformed charge,

$$
\mathrm{i}(s-1)\left[\mathcal{P}_{s}, \mathcal{Q}_{r}\right] \simeq-(s+r-2) \mathcal{Q}_{s+r-1} .
$$

Note that according to (2.15) both sides have a coincident range. In appendix A we prove (5.2) by using the generating equation (2.14). Consequently we can reduce the range by fixing the connection $\Gamma_{m, n}$ to

$$
\Gamma_{m, n}=\frac{n-1}{n-m} \Pi_{n-m+1} .
$$

Up to local similarity transformations, the connection (4.10) and the corresponding general deformation (4.3) then become

$$
\Xi=\tilde{\Pi}+\Upsilon, \quad \mathrm{d} \mathcal{Q}=\mathrm{i}\left[\tilde{\mathcal{P}}_{k}, \mathcal{Q}\right] \tilde{\Pi}_{k}+\mathrm{i}\left[\mathcal{Y}_{r, s}, \mathcal{Q}\right] \Upsilon_{r, s},
$$

where

$$
\tilde{\Pi}:=\Pi+\Gamma=\tilde{\mathcal{P}}_{k} \tilde{\Pi}_{k}, \quad \tilde{\Pi}_{k}=\frac{1}{k-1} \Pi_{k}, \quad \tilde{\mathcal{P}}_{k}:=(k-1) \mathcal{P}_{k}+\mathfrak{G}_{k} .
$$


The matrix $\mathfrak{G}_{k}$ is defined by ( $\left.\operatorname{cf}(3.37)\right)$

$$
\begin{aligned}
& \mathfrak{G}_{k}=\sum_{n}(n+k-2) \mathfrak{G}_{n, n+k-1}, \quad \text { that is } \\
& {\left[\mathfrak{G}_{k}, \mathcal{Q}\right]_{n}=-\mathrm{i}(n+k-2) \mathcal{Q}_{n+k-1} .}
\end{aligned}
$$

The factor $(k-1)$ in the definition of $\tilde{\mathcal{P}}_{k}$ and $\tilde{\Pi}_{k}$ is introduced for later convenience. Computationally, we find that the first-order deformation terms for the $\mathfrak{g l}(K)$ chain agree with those obtained in [24] and have the range

$$
\left|\mathrm{i}\left[\tilde{\mathcal{P}}_{k}^{(0)}, \mathcal{Q}_{r}^{(0)}\right]\right|=r+k-2 .
$$

In other words, deforming with $\tilde{\mathcal{P}}_{k}(0)$ increases the range by $k-2$. It appears that this is the minimal range one can achieve by correcting the boost deformation with the connection $\Gamma$.

Example. For the $\mathfrak{g l}(K)$ chain, the operators of range four in the first-order term $\mathcal{Q}_{2}^{(1)}(3.22)$ match the longest terms in the undeformed charge $\mathcal{Q}_{4}^{(0)}(2.16)$. With the choice (5.3), the new term $\mathcal{Q}_{2}^{(1)}$ becomes

$$
\begin{aligned}
\mathcal{Q}_{2}^{(1)}= & 2 \cdot \frac{1}{2}(-2[1]+2[2,1]-[2,3,4,1]+[2,4,1,3]+[3,1,4,2]-[4,1,2,3]) \\
& +3 \cdot \frac{1}{3}(-[1]+2[2,1]-[3,2,1]+[2,3,4,1]-[2,4,1,3]-[3,1,4,2]+[4,1,2,3]) \\
= & -3[1]+4[2,1]-[3,2,1] ;
\end{aligned}
$$

its range is reduced by one unit. It also agrees literally with the corresponding deformation in $[24,25]$.

\subsection{Boost connection at higher orders}

While the first-order terms of the boost deformation now have minimal range, the range of higher order terms in the expansion (4.5) can be further reduced. In particular, with the simplest choice $\tilde{\Pi}_{k}=\mathrm{d} \alpha_{k}$, we find that for a deformation along any path $\alpha_{k}(\lambda)$, the range of higher order terms is not additive in the powers of $\alpha_{k}(\lambda)$. For example the term of $\mathcal{Q}_{r=2}(\lambda)$ of the $\mathfrak{g l}(K)$ chain that is of order $\alpha_{3}(\lambda) \alpha_{4}(\lambda)$ has range $\operatorname{six}^{8}$; additivity would require it to have range five $(r+3)$, since the leading-order terms proportional to $\alpha_{3}(\lambda), \alpha_{4}(\lambda)$ have range three $(r+1)$ and four $(r+2)$, $\mathrm{cf}(5.7)$.

Experimentally, we find that the following expansion for the connection $\tilde{\Pi}_{k}$ minimizes the range:

$$
\tilde{\Pi}_{k}=\mathrm{d} \alpha_{k}+\sum_{M=1}^{\infty} \sum_{\ell_{1}, \ldots, \ell_{M}=3}^{\infty}\left(\prod_{j=1}^{M}\left(\ell_{j}-2\right) \alpha_{\ell_{j}}\right) \mathrm{d} \alpha_{k+M-\sum_{j} \ell_{j}},
$$

where we set $\mathrm{d} \alpha_{k<3}=0$. This choice also renders the range of higher order terms additive in the above sense. While this result appears ad hoc, we also found an equivalent implicit definition which makes a connection to earlier results: define first the function $u(x)$ as in [24]

$$
u(x)=x+\sum_{n=3}^{\infty} \frac{\alpha_{n}}{x^{n-2}},
$$

\footnotetext{
8 It is possible that a naive application of the recursion relation does not yield the desired range. Note, however, that we do not display explicitly deformations by local operators, which are required to reduce the length in most cases, see appendix B.
} 
and its inverse $x(u)=u+\mathcal{O}(\alpha)$. The connection $\tilde{\Pi}$ is then implicitly defined by the relation

$$
\mathrm{d} x(u)=-\sum_{n=3}^{\infty} \frac{\tilde{\Pi}_{n}}{x^{n-2}} .
$$

The relation should be interpreted in the following way: the function $x(u)$ depends implicitly on the moduli $\alpha_{k}$ and the differential acts on these only. Thus $\mathrm{d} x(u)$ is a function of $\alpha_{k}, \mathrm{~d} \alpha_{k}$ and $u$. Replacing the latter by the inverse function $u(x)$ and casting the result in the form on the right hand side of (5.11) defines the $\tilde{\Pi}_{n}$.

While the choice (5.11) seems not well motivated at first sight, we observe that the parametrization (5.9) gives the deformed charges a definite, canonical range pattern: For the $\mathfrak{g l}(K)$ chain, we find that with a given path $\alpha_{k}(\lambda)$ the range of each term in the expansion (4.5) depends additively on the moduli $\alpha_{k}(\lambda)$ in its coefficient-for each power of each $\alpha_{k}(\lambda)$, the range increases by $k-2$, cf (5.7). Furthermore, this range apparently is maximally reduced by (5.9) and does not depend on the specific path $\alpha(\lambda)$ in moduli space. As we will see below, the choice (5.9) moreover guarantees that the connection $\tilde{\Pi}$ is flat, which turns the deformed charges into proper functions on moduli space.

\subsection{Bilocal connection at higher orders}

It remains to consider the bilocal deformations: as long as we restrict to only bilocal deformations, i.e. $\Pi=\Gamma=0$, the simple choice

$$
\Upsilon_{r, s}=2 \mathrm{~d} \beta_{r, s}
$$

apparently results in a definite, minimal increase of the charges' ranges. Here, the factor of two is introduced for consistency with the existing literature. Our explicit computations for the $\mathfrak{g l}(K)$ chain show that with (5.12), as for the boost connection (5.9), the range of each term in the expansion (4.5) depends additively on the moduli $\beta_{r, s}(\lambda)$ in its coefficient. Here, each power of each $\beta_{r, s}(\lambda)$ increases the range by $s-1$; at leading order, the range equals

$$
\left|\mathrm{i}\left[\left[\mathcal{Q}_{r}^{(0)} \mid \mathcal{Q}_{s}^{(0)}\right], \mathcal{Q}_{t}^{(0)}\right]\right|=t+s-1 .
$$

Moreover, the range of all deformation terms is independent of the path $\beta(\lambda)$ in moduli space and apparently cannot be further reduced by a redefinition of $\mathcal{Y}_{r, s}$. As an example, the term of $\mathcal{Q}_{2}$ proportional to $\beta_{2,3}(\lambda)$ is given in (3.34), up to an overall factor.

If we, however, switch on the boost connection $\tilde{\Pi}$ with the parametrization (5.9) and include the change of basis (5.3), we find that the range of terms that come with mixed powers of $\alpha$ 's and $\beta$ 's is not minimal. Again, the example of the $\mathfrak{g l}(K)$ chain suggests that the range of each term is minimized by the choice (with $\beta_{r, s}=-\beta_{s, r}$ )

$$
\Upsilon_{r, s}=2 \mathrm{~d} \beta_{r, s}+2 \beta_{n, s} \Gamma_{n, r}+2 \beta_{r, n} \Gamma_{n, s} .
$$

With this choice, the range of mixed terms also becomes additive in the powers of each modulus $\alpha_{k}(\lambda), \beta_{r, s}(\lambda)$. As for the pure $\alpha$-terms and the pure $\beta$-terms, the range increases by $k-2$ for each power of $\alpha_{k}(\lambda)$ and by $s-1$ for each power of $\beta_{r, s}(\lambda)$.

Note that since $\Gamma_{r, s}$ is parametrized by the moduli $\alpha_{k}$, the prescription (5.14) changes (5.5) once more by a non-trivial term. Namely, $\tilde{\mathcal{P}}_{k}$ becomes

$$
\tilde{\mathcal{P}}_{k}=(k-1) \mathcal{P}_{k}+\mathfrak{G}_{k}+2 \mathrm{i} \beta_{r, s}\left[\left[\mathfrak{G}_{k}, \mathcal{Q}\right]_{r} \mid \mathcal{Q}_{s}\right]+\left[\mathcal{Q}_{r} \mid\left[\mathfrak{G}_{k}, \mathcal{Q}\right]_{s}\right]
$$

Examples for higher order terms of the expansion (4.5) are given in appendix B. 


\subsection{Flatness}

The redefinition (5.3) made in favor of a canonical interaction range spoils the flatness of the connection (4.8). As discussed in section 4, the boost and bilocal connection $\Pi+\Upsilon$ alone is flat $^{9}$, while a change of basis $\Gamma$ introduces curvature (4.22). As we will see in the following, flatness of the connection is restored by the parametrization constructed above.

Boost connection. With the definition (5.3), the flatness condition (4.25) for $\Gamma$ becomes

$$
\begin{aligned}
0 & =\mathrm{d} \Gamma_{m, n}+\Gamma_{m, k} \wedge \Gamma_{k, n} \\
& =(n-1) \mathrm{d} \tilde{\Pi}_{n-m+1}+\sum_{k}(k-1)(n-1) \tilde{\Pi}_{k-m+1} \wedge \tilde{\Pi}_{n-k+1}, \quad m, n \geqslant 2,
\end{aligned}
$$

which is not satisfied for generic $\tilde{\Pi}$. Note that for $m=1$, equation (5.16) becomes the boost flatness condition (4.23). Thus we can summarize both formulae if we extend the range of $m$ in (5.16) to $m \geqslant 1$. Setting

$$
n \rightarrow n+m-1, \quad k \rightarrow k+m-1
$$

the flatness conditions (4.23), (4.25) thus become

$$
0=\mathrm{d} \tilde{\Pi}_{n}+\sum_{k}(k+m-2) \tilde{\Pi}_{k} \wedge \tilde{\Pi}_{n-k+1}, \quad n \geqslant 2, m \geqslant 1 .
$$

If we define the connection $\tilde{\Pi}_{n}$ to be zero for $n<3$, we do not have to change the limits of the sum when shifting the indices. The antisymmetry of the wedge product in (5.18) implies that under the sum over $k$ the part with a constant coefficient vanishes. Hence, we have shown that equations (4.23), (4.25) for the choice (5.3) are actually independent of the index $m$ and reduce to

$$
0=\mathrm{d} \tilde{\Pi}_{n}+\sum_{k} k \tilde{\Pi}_{k} \wedge \tilde{\Pi}_{n-k+1}, \quad n \geqslant 2
$$

We now show that this flatness condition is satisfied by the definition (5.11). This can be seen as follows:

$$
\begin{aligned}
0 & =-\mathrm{dd} x(u)=\sum_{n=3}^{\infty}\left(\frac{\mathrm{d} \tilde{\Pi}_{n}}{x^{n-2}}-(n-2) \frac{\tilde{\Pi}_{n} \wedge \mathrm{d} x(u)}{x^{n-1}}\right) \\
& =\sum_{n=3}^{\infty}\left(\frac{\mathrm{d} \tilde{\Pi}_{n}}{x^{n-2}}+\sum_{k=3}^{\infty} \frac{(n-2) \tilde{\Pi}_{n} \wedge \tilde{\Pi}_{k}}{x^{n+k-3}}\right) \\
& =\sum_{n=3}^{\infty} \frac{1}{x^{n-2}}\left(\mathrm{~d} \tilde{\Pi}_{n}+\sum_{k=3}^{\infty} k \tilde{\Pi}_{k} \wedge \tilde{\Pi}_{n-k+1}\right),
\end{aligned}
$$

where going to the last line we have renamed the indices $k \leftrightarrow n$ in the second sum followed by an index shift $n \rightarrow n-k+1$. We furthermore dropped constant factors in front of the wedge product due to the antisymmetry and did not change the limits of the sum since $\tilde{\Pi}_{k<3}=0$ by definition. Now, (5.20) implies (5.19).

Bilocal connection. We now show that the remaining flatness condition (4.24) is satisfied by the parametrization (5.14), provided that $\Gamma$ fulfils (4.25). As we have seen above, this is the case for the choice (5.3). Plugging (5.14) into (4.24) yields

$$
\begin{aligned}
& \begin{aligned}
\frac{1}{2}\left(\mathrm{~d} \Upsilon_{r, s}+\Gamma_{p, r}\right. & \left.\wedge \Upsilon_{p, s}+\Gamma_{p, s} \wedge \Upsilon_{r, p}\right)=\mathrm{d} \beta_{n, s} \wedge \Gamma_{n, r}+\beta_{n, s} \mathrm{~d} \Gamma_{n, r}+\mathrm{d} \beta_{r, n} \wedge \Gamma_{n, s}+\beta_{r, n} \mathrm{~d} \Gamma_{n, s} \\
& +\Gamma_{p, r} \wedge \mathrm{d} \beta_{p, s}+\beta_{n, s} \Gamma_{p, r} \wedge \Gamma_{n, p}+\beta_{p, n} \Gamma_{p, r} \wedge \Gamma_{n, s} \\
& +\Gamma_{p, s} \wedge \mathrm{d} \beta_{r, p}+\beta_{n, p} \Gamma_{p, s} \wedge \Gamma_{n, r}+\beta_{r, n} \Gamma_{p, s} \wedge \Gamma_{n, p}=0
\end{aligned} \\
& 9
\end{aligned}
$$


where we have used equation (4.25). Hence, the definition (5.14) leads to a flat bilocal connection in the sense that (4.24) is satisfied.

\subsection{Summary}

To summarize, the parametrization (5.3), (5.11), (5.14),

$$
\begin{aligned}
& \Gamma_{r, s}=\frac{s-1}{s-r} \Pi_{s-r+1}, \\
& \sum_{n=3}^{\infty} \frac{\Pi_{n}\left(\alpha_{m}\right)}{(n-1) x^{n-2}}=-\mathrm{d} x(u), \\
& \Upsilon_{r, s}=2 \mathrm{~d} \beta_{r, s}+2 \beta_{n, s} \Gamma_{n, r}+2 \beta_{r, n} \Gamma_{n, s},
\end{aligned}
$$

results in deformed charges $\mathcal{Q}_{r}$ with a definite pattern of ranges. Moreover, this parametrization renders the connection (4.8) flat. Hence the charges are proper functions of the moduli space parameters $\alpha, \beta$ up to local similarity transformations

$$
\mathcal{Q}_{n}=\mathcal{Q}_{n}^{(0)}+\alpha_{k} \mathcal{Q}_{n}^{[k]}+\beta_{r, s} \mathcal{Q}_{n}^{[r \mid s]}+\alpha_{k} \alpha_{l} \mathcal{Q}_{n}^{[k, l]}+\alpha_{k} \beta_{r, s} \mathcal{Q}_{n}^{[k, r \mid s]}+\beta_{r, s} \beta_{t, u} \mathcal{Q}_{n}^{[r|s, t| u]}+\cdots
$$

The individual terms $\mathcal{Q}_{n}^{[\ldots]}$ in the $\mathfrak{g l}(K)$ case agree with those obtained in $[24,25]^{10}$. As discussed in the previous subsections, they have an interaction range

$$
\left|\mathcal{Q}_{n}^{\left[k_{1}, \ldots, k_{t}, r_{1}\left|s_{1}, \ldots, r_{u}\right| s_{u}\right]}\right|=n+\sum_{\ell=1}^{t}\left(k_{\ell}-2\right)+\sum_{\ell=1}^{u}\left(s_{\ell}-1\right),
$$

i.e. each boost deformation $\tilde{\mathcal{P}}_{k}$ increases the range by $k-2$ and each bilocal deformation $\mathcal{Y}_{r, s}$ increases the range by $s-1$.

Note that the definition (5.3) fixes a specific change of basis that accompanies the boost deformation. This specific choice minimizes the ranges of the deformed charges $\mathcal{Q}$ and renders the connection flat. The charges $\mathcal{Q}$ thus form a canonical basis of the space of charges at each point in moduli space. They reproduce the normalized charges $\overline{\mathcal{Q}}_{r}$ of [24] and depend only on the reduced set of moduli $\left\{\alpha_{k}, \beta_{r, s}, \varepsilon_{l}\right\}$. However, nothing prevents us from choosing a different basis

$$
\tilde{\mathcal{Q}}_{m}=\gamma_{m, n} \mathcal{Q}_{n}
$$

for the space of charges after the charges have been deformed. For comparison with [24], note that the symbols $\left\{\mathcal{Q}, \tilde{\mathcal{Q}}, \alpha_{k}, \beta_{r, s}, \gamma_{r, s}\right\}$ here correspond to the quantities $\left\{\overline{\mathcal{Q}}, \mathcal{Q}, \alpha_{k-3}\right.$, $\left.\beta_{r, s}, \gamma_{r, s}\right\}$ there.

\subsection{Properties of deformations}

We have seen that the interaction ranges of the deformed charges obey a certain pattern (5.24). In this subsection we discuss additional properties of the deformations as well as their relation to gauge theory.

Parity. We introduce a parity operator $P$ acting on local, boost and bilocal operators. For a local charge of manifest parity we then have

$$
P \mathcal{Q}_{r} P^{-1}=(-1)^{p_{r}} \mathcal{Q}_{r},
$$

${ }^{10}$ Some charge terms $\mathcal{Q}_{n}^{[\ldots]}$ are calculated explicitly in appendix B. 
where $p_{r}$ is even or odd for $\mathcal{Q}_{r}$ being even or odd under parity. Since we only consider bilocal charges within commutators with local charges, we can make use of the fact that due to (3.25) we have

$$
\left[\left[\mathcal{Q}_{r} \mid \mathcal{Q}_{s}\right], \mathcal{Q}_{t}\right]=-\left[\left[\mathcal{Q}_{s} \mid \mathcal{Q}_{r}\right], \mathcal{Q}_{t}\right] .
$$

Further using that $P\left[\mathcal{Q}_{r} \mid \mathcal{Q}_{s}\right] P^{-1}=\left[P \mathcal{Q}_{s} P^{-1} \mid P \mathcal{Q}_{r} P^{-1}\right]$ due to (3.24), we find

$$
\begin{aligned}
P\left[\left[\mathcal{Q}_{r} \mid \mathcal{Q}_{s}\right], \mathcal{Q}_{t}\right] P^{-1} & =\left[P\left[\mathcal{Q}_{r} \mid \mathcal{Q}_{s}\right] P^{-1}, P \mathcal{Q}_{t} P^{-1}\right] \\
& =(-1)^{p_{s}+p_{r}+p_{t}}\left[\left[\mathcal{Q}_{s} \mid \mathcal{Q}_{r}\right], \mathcal{Q}_{t}\right] \\
& =(-1)^{p_{s}+p_{r}+p_{t}-1}\left[\left[\mathcal{Q}_{r} \mid \mathcal{Q}_{s}\right], \mathcal{Q}_{t}\right]
\end{aligned}
$$

The interpretation (3.32) of boost operators in terms of bilocal operators implies that

$$
P\left[\mathcal{B}\left[\mathcal{Q}_{k}\right], \mathcal{Q}_{r}\right] P^{-1}=(-1)^{p_{k}+p_{r}-1}\left[\mathcal{B}\left[\mathcal{Q}_{k}\right], \mathcal{Q}_{r}\right] .
$$

Assuming that the undeformed Hamiltonian $\mathcal{Q}_{2}^{(0)}$ has even parity, equation (2.14) then implies that the undeformed even and odd charges $\mathcal{Q}_{2 r}^{(0)}$ and $\mathcal{Q}_{2 r+1}^{(0)}$ have even and odd parity, respectively. According to (5.28), (5.29) the long-range deformations can be classified according to

$P \mathcal{Q}_{t}^{\left[k_{1}, \ldots, k_{t}, r_{1}\left|s_{1}, \ldots, r_{u}\right| s_{u}\right]} P^{-1}=(-1)^{n+\sum_{\ell=1}^{t}\left(k_{\ell}-1\right)+\sum_{\ell=1}^{u}\left(r_{\ell}+s_{\ell}-1\right)} \mathcal{Q}_{t}^{\left[k_{1}, \ldots, k_{t}, r_{1}\left|s_{1}, \ldots, r_{u}\right| s_{u}\right]}$.

Number of crossings for fundamental $\mathfrak{g}=\mathfrak{g l}(K)$. Considering the fundamental $\mathfrak{g l}(K)$ spin chain, the building blocks of invariant operators are given by simple permutations (2.9). One characteristic quantity of the interaction terms is the number of elementary permutations (crossings) $\rangle \mathcal{L}_{k}\left\langle\right.$ contained in an operator $\mathcal{L}_{k}$, e.g.

$$
\begin{gathered}
\rangle[2,1]+[2,1,4,3]\langle=2 \\
\text { X X X }
\end{gathered}
$$

As shall be explained below, these numbers are a relevant quantity for the gauge theory, because of their relation to the minimum perturbative order at which they may appear [3, 31]. Note that as for the range $\left|\mathcal{L}_{k}\right|$ of a linear combination of local operators we define $\rangle \mathcal{L}_{k}\langle$ to be the maximum number of crossings.

In commutators of boost and local operators we add the numbers of crossings of the boost operator $\mathcal{B}\left[\mathcal{L}_{k}\right]$ and the local operator $\mathcal{L}_{l}$. Equation (2.14) implies that the leading-order charges $\mathcal{Q}_{r}^{(0)}$ have $r-1$ crossings

$$
\mid \mathcal{Q}_{r}^{(0)}\langle=r-1
$$

Consequently, the number of crossings of a boost deformation $\left[\mathcal{P}_{k}^{(0)}, \mathcal{Q}_{r}^{(0)}\right]$ has $r+k-2$ crossings

$$
\backslash\left[\mathcal{P}_{k}^{(0)}, \mathcal{Q}_{r}^{(0)}\right]\langle=r+k-2 .
$$

Adding a term proportional to $\mathcal{Q}_{r+k-1}^{(0)}$ with $r+k-2$ crossings for the minimal interaction range (5.4) apparently does not reduce the crossings. Hence, the structure $\mathcal{Q}_{r}^{[k]}$ multiplied by $\alpha_{k}$ generically contains $r+k-2$ crossings. More generally, the number of crossings increases by $k-1$ for each power of $\alpha_{k}$.

Also for bilocal deformations, the number of crossings of the bilocal operator adds up with the number of crossings of the deformed structure to give the number of crossings of the result. The commutator of a bilocal operator $\left[\mathcal{Q}_{r}^{(0)} \mid \mathcal{Q}_{s}^{(0)}\right]$ which has $r+s-2$ crossings and a charge $\mathcal{Q}_{t}^{(0)}$ with $t-1$ crossings thus has $t+r+s-3$ crossings

$$
\mid\left[\left[\mathcal{Q}_{r}^{(0)} \mid \mathcal{Q}_{s}^{(0)}\right], \mathcal{Q}_{t}^{(0)}\right]\langle=t+r+s-3 .
$$


The number of crossings of a general deformation term is therefore given by

$$
\left|\mathcal{Q}_{t}^{\left[k_{1}, \ldots, k_{t}, r_{1}\left|s_{1}, \ldots, r_{u}\right| s_{u}\right]}\right|=t-1+\sum_{\ell=1}^{u}\left(r_{\ell}+s_{\ell}-2\right)+\sum_{\ell=1}^{t}\left(k_{\ell}-1\right),
$$

in full agreement with the prediction of [24].

Number of crossings for $\mathfrak{g}=\mathfrak{g l}(2)$. A particularly interesting case is given for $\mathfrak{g l}(2)$ symmetry which represents the $\mathfrak{s u}(2)$ sector spin chain of $\mathcal{N}=4$ SYM theory. We observe that for $\mathfrak{g l}(2)$ the anti-symmetrizer of $2+1$ spins vanishes

$$
0=\sum_{i, j, k \in\{1,2,3\}} \varepsilon^{i j k}[i, j, k] .
$$

This allows us to reduce the number of crossings of boost deformations. Consider for instance the Hamiltonian structure proportional to $\alpha_{3}$ :

$$
\mathcal{Q}_{2}^{[3]}=-3[1]+4[2,1]-[3,2,1]
$$

Here we can replace the term with three vertices by permutations with at most two vertices

$$
\begin{aligned}
& {[3,2,1]=[2,3,1]+[3,1,2]-2[2,1]+[1] \text {. }} \\
& X=X X+X-2 X+1
\end{aligned}
$$

Note that we have performed boundary identifications. Hence, the operator structure $\mathcal{Q}_{2}^{[3]}$ in fact contains only two elementary permutations [3]. It appears that one can reduce the number of crossings of other boost deformations in a similar fashion. Also the terms multiplied by higher powers of $\alpha_{k}$ appear to be regularizable in this way [24]. This observation for $\mathfrak{g}=\mathfrak{g l}(2)$ suggests a modification of (5.35) to

$$
\left.\backslash \mathcal{Q}_{t}^{\left[k_{1}, \ldots, k_{t}, r_{1}\left|s_{1}, \ldots, r_{u}\right| s_{u}\right]}\right|_{\mathfrak{g l}(2)}=t-1+\sum_{\ell=1}^{u}\left(r_{\ell}+s_{\ell}-2\right)+\sum_{\ell=1}^{t}\left(k_{\ell}-2\right) .
$$

The number of crossings for bilocal deformations does not decrease.

Parameter restrictions from the $\mathfrak{s u ( 2 ) ~ s e c t o r ~ o f ~} \mathcal{N}=4$ super Yang-Mills theory. Here we consider gauge theory restrictions on the $\mathfrak{g l}(2)$ spin chain charges based on their number of crossings [3, 31]. The integrable Hamiltonian appearing in the $\mathfrak{s u}(2)$ sector of $\mathcal{N}=4 \mathrm{SYM}$ theory obeys a fundamental principle: its number of crossings does not exceed the order of the coupling due to the correspondence to Feynman diagrams. As we will see, this requirement can be satisfied if one restricts the starting order of the moduli $\left\{\alpha_{k}(\lambda), \beta_{r, s}(\lambda)\right\}$ as a Taylor series in $\lambda$.

Within a $\mathfrak{g l}(K)$ invariant operator, an elementary permutation between two spin chain sites corresponds to a quartic scalar vertex of a Feynman graph in planar gauge theory. The interaction $\sim \lambda\left[\Phi_{i}, \Phi_{j}\right]^{2}$ in the $\mathcal{N}=4$ Lagrangian (cf [2]) translates to the fact that each elementary permutation comes with a factor of the coupling

$$
X \sim \lambda
$$

The number of Feynman vertices in the gauge theory Hamiltonian $\mathcal{H}(\lambda)$ must therefore equal the corresponding power of the coupling constant in perturbation theory. Translating this to 
the spin chain picture one has to take into account that the Hamiltonian $\mathcal{H}(\lambda)$ is shifted by one power of $\lambda$

$$
\mathcal{H}(\lambda)=\mathcal{H}_{0}+\lambda \mathcal{Q}_{2}(\lambda)
$$

Thus, an operator structure with $C$ crossings is allowed to contribute to $\mathcal{Q}_{2}(\lambda)$ at $\mathcal{O}\left(\lambda^{C-1}\right)$. Comparison to the formula for the number of crossings of our generated charge terms (5.39) shows that one has to restrict the parameter functions as in [24]

$$
\begin{aligned}
& \alpha_{k}(\lambda)=\mathcal{O}\left(\lambda^{k-2}\right), \\
& \beta_{r, s}(\lambda)=\mathcal{O}\left(\lambda^{r+s-2}\right) .
\end{aligned}
$$

With this prescription the ranges of the deformed charges grow by at most one site with each power of the coupling. The Hamiltonian at order $\lambda^{N}$ acts on no more than $N+1$ sites.

The long-range Hamiltonian $\mathcal{Q}_{2}(\lambda)$ for the $\mathfrak{g l}(K)$ chain in terms of permutation symbols and the coefficients $\alpha_{k}(\lambda), \beta_{r, s}(\lambda)$ including terms of order $\mathcal{O}\left(\lambda^{3}\right)$ is printed at the end of this paper in table 2 .

\section{Long-range Bethe ansatz}

We will now consider the asymptotic spectrum of a conserved charge on finite periodic chains. So far, the charges of the integrable model have been defined for infinite chains only. For a finite chain we demand that a particular charge matches with the integrable charge $\mathcal{Q}_{r}$ for all terms whose range does not exceed the length of the chain. This provides a proper definition of commuting long-range charges on finite chains up to a certain order in the power series. The asymptotic spectrum is the spectrum in the form of a power series modulo terms of higher orders where the operator is not uniquely specified by the above definition. In particular, the longer the chain, the higher the order at which the asymptotic spectrum truncates.

We will use the asymptotic Bethe ansatz $[32,33]$ to determine three basic observables of asymptotic states on the infinite chain: vacuum charge density, magnon dispersion relation and scattering matrix. These data are sufficient to set up the resulting asymptotic Bethe equations providing the asymptotic spectrum of conserved charges on finite periodic chains. We shall use the full set of deformations found in section 3 and the corresponding moduli defined in section 5, and our result will turn out to agree with the earlier proposal in [24].

In the first parts of this section we shall assume a $\mathfrak{s l}(2)$ or $\mathfrak{s u}(2)$ spin chain with spin $t / 2$ representations on all sites, i.e. a long-range Heisenberg $\mathrm{XXX}_{t / 2}$ model. Later we will generalize the results to higher rank symmetry algebras $\mathfrak{g}$.

\subsection{Ferromagnetic vacuum}

The ferromagnetic vacuum $|0\rangle$ is a pure state in which all spins are aligned to have an identical orientation $|\mathrm{HW}\rangle$ being of highest weight w.r.t. the symmetry algebra

$$
|0\rangle=\cdots \otimes|\mathrm{HW}\rangle \otimes|\mathrm{HW}\rangle \otimes|\mathrm{HW}\rangle \otimes \cdots .
$$

Furthermore this state is assumed to be an eigenstate of $\mathcal{Q}_{r}^{\mathrm{NN}}$ with vanishing eigenvalue (density). The latter property can always be achieved by subtracting from $\mathcal{Q}_{r}^{\mathrm{NN}}$ the length operator $\mathcal{N}$ multiplied by the eigenvalue density.

The long-range spin chain is a deformation of the nearest neighbor model induced by equation (3.1). This equation is of parallel transport type implying that merely the eigenvectors are deformed but not the spectrum. Here also the state remains undeformed because the deformations respect the symmetry and there is only a single highest weight state. Altogether, the vacuum density of all deformed $\mathcal{Q}_{r}$ is zero. 


\subsection{One-magnon states and dispersion relations $(\alpha)$}

One-magnon states are excitations of the ferromagnetic vacuum (6.1) where one spin is replaced by a next-to-highest-weight state $|\mathrm{NHW}\rangle$. Let the spin at position $k$ be flipped

$$
|k\rangle=\cdots \otimes|\mathrm{HW}\rangle \otimes|\mathrm{NHW}\rangle \otimes|\mathrm{HW}\rangle \otimes \cdots .
$$

The magnon state $|p\rangle$ is a state with definite momentum $p$ along the chain

$$
|p\rangle=\sum_{k} \mathrm{e}^{\mathrm{i} p k}|k\rangle
$$

Magnons are eigenstates of the charges $\mathcal{Q}_{r}$ because the latter are homogeneous local operators. The eigenvalue of $\mathcal{Q}_{r}$ on $|p\rangle$ is called the dispersion relation $q_{r}(p)$. We will now study how the dispersion relation $q_{r}$ ( $p$ ) changes under the deformation (3.1). Although the latter does not change the spectrum, it can deform the eigenstate to one with a different momentum $p$. Let us therefore act with the various deformations on a magnon state. Local operators (2.9) acting on this state are equivalent to linear combinations of shift operators

$$
\mathcal{U}^{j}:|k\rangle \mapsto|k-j\rangle,
$$

whose action in momentum space is given by

$$
\mathcal{U}^{j}|p\rangle=\mathrm{e}^{\mathrm{i} p j}|p\rangle \text {. }
$$

Thus local deformations conserve the momentum. Likewise boost operators can be represented by boosted shift operators $\mathcal{B}\left[\mathcal{U}^{j}\right]$ which act on one-magnon states as

$$
\mathcal{B}\left[\mathcal{U}^{j}\right]|p\rangle=\sum_{k} k \mathrm{e}^{\mathrm{i} p k}|k-j\rangle=\sum_{k}(k+j) \mathrm{e}^{\mathrm{i} p(k+j)}|k\rangle=-\mathrm{i} \frac{\partial}{\partial p}\left(\mathrm{e}^{\mathrm{i} p j}|p\rangle\right)
$$

This shows that boost operators change the momentum of a magnon state. Conversely, for bilocal charges $\left[\mathcal{Q}_{r} \mid \mathcal{Q}_{s}\right]$ each elementary charge will annihilate the state due to a vanishing vacuum charge density (see above) unless it acts on the flipped spin. Therefore the only non-trivial contributions come from where both charges overlap with the flipped spin. This is equivalent to the action of a local operator and thus it cannot change the momentum of the magnon state.

Let us now act with the deformation equations (4.6), (4.7), (4.8) on the one-magnon state $|p\rangle$. The only relevant contributions come from boost and change of basis deformations, which have been combined into $\tilde{\mathcal{P}}_{k}$ in section 5

$$
\mathrm{d} \mathcal{Q}=\mathrm{i}\left[\tilde{\mathcal{P}}_{k}, \mathcal{Q}\right] \tilde{\Pi}_{k}=\sum_{k=3}^{\infty} \mathrm{i}\left[(k-1) \mathcal{P}_{k}+\mathfrak{G}_{k}, \mathcal{Q}\right] \tilde{\Pi}_{k}
$$

For $\left[\mathcal{P}_{j}, \mathcal{Q}_{\ell}\right]=\left[\mathcal{B}\left[\mathcal{Q}_{j}\right], \mathcal{Q}_{\ell}\right]$ we need to compute the commutator of a boosted shift with a shift operator

$\left[\mathcal{B}\left[\mathcal{U}^{j}\right], \mathcal{U}^{\ell}\right]|p\rangle=\mathrm{i} \frac{\partial}{\partial p}\left(\mathrm{e}^{\mathrm{i} p j} \mathrm{e}^{\mathrm{i} p \ell}|p\rangle\right)-\mathrm{i} \mathrm{e}^{\mathrm{i} p \ell} \frac{\partial}{\partial p}\left(\mathrm{e}^{\mathrm{i} p j}|p\rangle\right)=\mathrm{i} \mathrm{e}^{\mathrm{i} p j}\left(\frac{\partial}{\partial p} \mathrm{e}^{\mathrm{i} p \ell}\right)|p\rangle$,

such that the commutator of a boosted charge $\mathcal{B}\left[\mathcal{Q}_{r}\right]$ with a charge $\mathcal{Q}_{s}$ acts on a one-magnon state as

$$
\left[\mathcal{B}\left[\mathcal{Q}_{r}\right], \mathcal{Q}_{s}\right]|p\rangle=\mathrm{i} q_{r}(p) \frac{\partial q_{s}(p)}{\partial p}|p\rangle .
$$

This implies the following differential equation for the one-magnon eigenvalues $q_{r}$

$$
\mathrm{d} q_{r}(p)=\sum_{k=3}^{\infty}\left(-(k-1) q_{k} \frac{\partial q_{r}}{\partial p}+(k+r-2) q_{r+k-1}\right) \tilde{\Pi}_{k} .
$$


We now prove that the solution to the above differential equation (6.10) is given by the well-known form of the one-magnon eigenvalues [6]

$$
q_{r}(t, u)=\frac{\mathrm{i}}{n-1}\left(\frac{1}{x\left(u+\frac{\mathrm{i}}{2} t\right)^{r-1}}-\frac{1}{x\left(u-\frac{\mathrm{i}}{2} t\right)^{r-1}}\right) .
$$

The rapidity map $x(u)=u+\mathcal{O}\left(\alpha_{k}\right)$ was introduced in (5.10). The map between the momentum $p$ and the rapidity $u(p)$ is implicitly defined through

$$
\exp (\mathrm{i} p(t, u))=\frac{x\left(u+\frac{\mathrm{i}}{2} t\right)}{x\left(u-\frac{\mathrm{i}}{2} t\right)} \text {. }
$$

Note that $t$ is a constant of integration that can be freely chosen in the above equations ${ }^{11}$. We now rewrite the above differential equation using the parameter $u$ instead of the momentum $p$. For the differential of $q_{r}$ this implies

$$
\mathrm{d} q_{r}(u)=\mathrm{d} q_{r}(p)+\frac{\partial q_{r}}{\partial p} \mathrm{~d} p(u) .
$$

Here, the differential operator $\mathrm{d}$ acts only on the $\xi_{j}$ on which the functions $q_{r}(u), q_{r}(p)$ explicitly depend. That is $u$ is held fixed in $\mathrm{d} q_{r}(u)$ while $p$ is held fixed in $\mathrm{d} q_{r}(p)$, as is the case in (6.10). Using the defining equation of $\tilde{\Pi}_{k}(5.11)$ and (6.11), (6.12), we can compute and simplify $\mathrm{d} p(u), \mathrm{d} q_{r}(u)$

$$
\begin{aligned}
& \mathrm{d} p(u)=-\mathrm{i}\left(\frac{\mathrm{d} x\left(u+\frac{\mathrm{i}}{2} t\right)}{x\left(u+\frac{\mathrm{i}}{2} t\right)}-\frac{\mathrm{d} x\left(u-\frac{\mathrm{i}}{2} t\right)}{x\left(u-\frac{\mathrm{i}}{2} t\right)}\right)=\sum_{k=3}^{\infty}(k-1) \tilde{\Pi}_{k} q_{k}, \\
& \mathrm{~d} q_{r}(u)=-\mathrm{i}\left(\frac{\mathrm{d} x\left(u+\frac{\mathrm{i}}{2} t\right)}{x\left(u+\frac{\mathrm{i}}{2} t\right)^{r}}-\frac{\mathrm{d} x\left(u-\frac{\mathrm{i}}{2} t\right)}{x\left(u-\frac{\mathrm{i}}{2} t\right)^{r}}\right)=\sum_{k=3}^{\infty}(r+k-2) \tilde{\Pi}_{k} q_{r+k-1} .
\end{aligned}
$$

The result for $\mathrm{d} p(u)$ can also be obtained from the result for $\mathrm{d} q_{r}(u)$ by interpreting the momentum operator as the first charge $p=q_{1}$. Plugging (6.15), (6.14) into (6.13) then yields the differential equation (6.10), which shows that $p(u), q_{r}(u)$ provides the correct long-range charge eigenvalues.

At this point we can observe and disentangle the effect of the boost and basis rotation in (6.10): the boosts are responsible for a deformation of the momentum function $p(u)$ while the deformations of the function $q_{r}(u)$ are caused solely by a change of basis.

To understand the role of the integration constant $t$, let us turn off the deformation moduli $\alpha_{k}=0$ such that $x(u)=u$ and consider the resulting dispersion relations (6.11), (6.12)

$$
\exp \left(\mathrm{i} p^{\mathrm{NN}}(t, u)\right)=\frac{u+\frac{\mathrm{i}}{2} t}{u-\frac{\mathrm{i}}{2} t}, \quad q_{r}^{\mathrm{NN}}(t, u)=\frac{\mathrm{i}}{r-1}\left(\frac{1}{\left(u+\frac{\mathrm{i}}{2} t\right)^{r-1}}-\frac{1}{\left(u-\frac{\mathrm{i}}{2} t\right)^{r-1}}\right) \text {. }
$$

It is well known that the functions $p^{\mathrm{NN}}(t, u), q_{r}^{\mathrm{NN}}(t, u)$ define the dispersion relation for a Bethe root $u$ where $t / 2$ is the spin label of the spin representation.

\subsection{Two-magnon states and scattering $(\beta)$}

Two-magnon states are states where the spin has been flipped at two positions

$$
|k, \ell\rangle=\cdots \otimes \mid \begin{array}{cc}
k & \downarrow \\
\downarrow & \downarrow \\
\downarrow
\end{array}
$$

${ }^{11}$ Any other linear combination of terms with different values of $t$ is permissible in $p, q_{r}$ as well. 
When the two spins are far enough apart, i.e. when the range of the Hamiltonian is smaller than their separation, it is safe to assume partial momentum eigenstates

$$
\left|p<p^{\prime}\right\rangle=\sum_{k \ll \ell} \mathrm{e}^{\mathrm{i} p k+i p^{\prime} \ell}|k, l\rangle .
$$

This state describes two magnons of momenta $p, p^{\prime}$ in the asymptotic region where the magnon with momentum $p$ is to the left of the magnon with momentum $p^{\prime}$. A two-magnon scattering state $\left|p, p^{\prime}\right\rangle$ can be written as a linear combination of the two asymptotic regions $\left|p<p^{\prime}\right\rangle$ and $\left|p^{\prime}<p\right\rangle$

$$
\left|p, p^{\prime}\right\rangle \simeq A\left(p, p^{\prime}\right)\left|p<p^{\prime}\right\rangle+A\left(p^{\prime}, p\right)\left|p^{\prime}<p\right\rangle .
$$

This expression is valid in the IR, we have not paid attention to UV terms $\sum_{k \approx k^{\prime}}\left|k, k^{\prime}\right\rangle$ where the two magnons are nearby. These terms are needed in the computation of the scattering factor which relates the phase in the two asymptotic regions

$$
S\left(p, p^{\prime}\right)=\frac{A\left(p^{\prime}, p\right)}{A\left(p, p^{\prime}\right)}
$$

Consequently, the scattering factor summarizes the effect of local interactions between the spins on IR physics, and it is the quantity that we need to determine.

Before we continue, let us make a change to the labels of magnon states. It is useful to replace the momentum $p$ by the rapidity $u(p)$ and define a magnon state with definite rapidity

$$
|u\rangle=F(u)|p(u)\rangle,
$$

where $F(u)$ is a convenient normalization factor. The main difference between $|p\rangle$ and $|u\rangle$ is that the former depends only on $p$ while the latter depends on $u$ and implicitly on the moduli. We postulate a differential equation for the normalization factor

$$
\frac{d F(u)}{F(u)}|u\rangle=\Pi_{k} \frac{\partial q_{k}}{\partial p}|u\rangle+\mathrm{i} \Lambda_{k} \mathcal{L}_{k}|u\rangle .
$$

Using (6.14), (6.6) one finds a simple differential equation for the state

$$
\mathrm{d}|u\rangle=\mathrm{d} F(u)|p\rangle+F(u) \mathrm{d} p(u) \frac{\partial}{\partial p}|p\rangle=\mathrm{i}\left(\Pi_{k} \mathcal{P}_{k}+\Lambda_{k} \mathcal{L}_{k}\right)|u\rangle=\mathrm{i}(\Pi+\Lambda)|u\rangle .
$$

It is useful in so far as to cancel the effect of local, boost and basis change deformations in the differential equations (4.6)-(4.8) acting upon the eigenvalue equation for $\mathcal{Q}$

$$
\begin{aligned}
0 & =\mathrm{d}(\mathcal{Q}|u\rangle-q(u)|u\rangle)=\mathrm{d} \mathcal{Q}|u\rangle+\mathcal{Q} \mathrm{d}|u\rangle-\mathrm{d} q(u)|u\rangle-q(u) \mathrm{d}|u\rangle \\
& =\mathrm{i}[\Xi, \mathcal{Q}]|u\rangle-\mathrm{d} q(u)|u\rangle+\mathrm{i} \mathcal{Q}(\Pi+\Lambda)|u\rangle-\mathrm{i} q(u)(\Pi+\Lambda)|u\rangle \\
& =\mathrm{i}[\Xi-\Pi-\Lambda, \mathcal{Q}]|u\rangle-\mathrm{d} q(u)|u\rangle \\
& =\mathrm{i}[\Xi-\Pi-\Gamma-\Lambda, \mathcal{Q}]|u\rangle=\mathrm{i}[\Upsilon, \mathcal{Q}]|u\rangle,
\end{aligned}
$$

where in the last line we made use of (6.15). We are thus left with only bilocal deformations $\Upsilon$.

Consider now the two-magnon scattering state with the corresponding eigenvalue equation (we discard contributions where the two magnons are close)

$$
\begin{aligned}
& \left|u, u^{\prime}\right\rangle \simeq A\left(u, u^{\prime}\right)\left|u<u^{\prime}\right\rangle+A\left(u^{\prime}, u\right)\left|u^{\prime}<u\right\rangle, \\
& \mathcal{Q}\left|u, u^{\prime}\right\rangle=\left(q(u)+q\left(u^{\prime}\right)\right)\left|u, u^{\prime}\right\rangle .
\end{aligned}
$$


Differentiating the eigenvalue equation we are led to the following equation:

$$
\begin{aligned}
& \begin{array}{l}
0 \mathrm{~d}\left[\left(\mathcal{Q}-q(u)-q\left(u^{\prime}\right)\right)\left|u, u^{\prime}\right\rangle\right] \\
=\mathrm{d} \mathcal{Q}\left|u, u^{\prime}\right\rangle \\
\quad-\mathrm{d} q(u)\left|u, u^{\prime}\right\rangle-\mathrm{d} q\left(u^{\prime}\right)\left|u, u^{\prime}\right\rangle \\
\quad+\left(\mathcal{Q}-q(u)-q\left(u^{\prime}\right)\right)\left(A\left(u, u^{\prime}\right) \mathrm{d}\left|u<u^{\prime}\right\rangle+A\left(u^{\prime}, u\right) \mathrm{d}\left|u^{\prime}<u\right\rangle\right) \\
\quad+\left(\mathcal{Q}-q(u)-q\left(u^{\prime}\right)\right)\left(\mathrm{d} A\left(u, u^{\prime}\right)\left|u<u^{\prime}\right\rangle+\mathrm{d} A\left(u^{\prime}, u\right)\left|u^{\prime}<u\right\rangle\right)
\end{array} \\
& =\mathrm{i}[\Upsilon,] Q\left|u, u^{\prime}\right\rangle+\left(\mathcal{Q}-q(u)-q\left(u^{\prime}\right)\right)\left(\mathrm{d} A\left(u, u^{\prime}\right)\left|u<u^{\prime}\right\rangle+\mathrm{d} A\left(u^{\prime}, u\right)\left|u^{\prime}<u\right\rangle\right) .
\end{aligned}
$$

Here we used the equations

$$
\begin{aligned}
& \left(\mathrm{d} q(u)+\mathrm{d} q\left(u^{\prime}\right)\right)\left|u, u^{\prime}\right\rangle=\mathrm{i}[\Gamma, \mathcal{Q}]\left|u, u^{\prime}\right\rangle, \\
& \mathrm{d}\left|u\left\langle u^{\prime}\right\rangle=\mathrm{i}(\Pi+\Lambda)\right| u\left\langle u^{\prime}\right\rangle,
\end{aligned}
$$

which are generalizations of equations (6.15), (6.23) and are valid up to UV terms in which the two magnons are close. Again the effect of the boost, local and basis change deformations cancels out and we are left with a differential equation for the prefactor $A\left(u, u^{\prime}\right)$ which only depends on the bilocal deformations

$$
\frac{\mathrm{d} A\left(u, u^{\prime}\right)}{A\left(u, u^{\prime}\right)}\left|u<u^{\prime}\right\rangle=\mathrm{i} \Upsilon\left|u<u^{\prime}\right\rangle=\mathrm{i}_{r, s} \mathcal{Y}_{r, s}\left|u<u^{\prime}\right\rangle
$$

Partial two-magnon states are obviously eigenstates of bilocal charges

$$
\left[\mathcal{Q}_{r} \mid \mathcal{Q}_{s}\right]\left|u<u^{\prime}\right\rangle=\left(q_{r}(u) q_{s}\left(u^{\prime}\right)+f_{r, s}(u)+f_{r, s}\left(u^{\prime}\right)\right)\left|u<u^{\prime}\right\rangle,
$$

where $f_{r, s}$ is a local contribution from both local charges acting on one of the two magnons. The differential equation for the prefactor $A$ has the simple solution

$$
A\left(u, u^{\prime}\right)=\exp \left[2 \mathrm{i} \beta_{r, s}\left(q_{r}(u) q_{s}\left(u^{\prime}\right)+f_{r, s}(u)+f_{r, s}\left(u^{\prime}\right)\right)\right] A_{0}\left(u, u^{\prime}\right) .
$$

For the scattering factor $S\left(u, u^{\prime}\right)=A\left(u^{\prime}, u\right) / A\left(u, u^{\prime}\right)$ it implies the deformation

$$
S\left(u, u^{\prime}\right)=\exp \left(-2 \mathrm{i} \theta\left(u, u^{\prime}\right)\right) S^{\mathrm{NN}}\left(u-u^{\prime}\right), \quad S^{\mathrm{NN}}\left(u-u^{\prime}\right)=\frac{u-u^{\prime}-\mathrm{i}}{u-u^{\prime}+\mathrm{i}}
$$

where $S^{\mathrm{NN}}\left(u, u^{\prime}\right)$ is the undeformed scattering factor and $\theta\left(u, u^{\prime}\right)$ is an overall scattering phase, the so-called dressing phase

$$
\theta\left(u, u^{\prime}\right)=\sum_{s>r=2}^{\infty} \beta_{r, s}\left(q_{r}(u) q_{s}\left(u^{\prime}\right)-q_{s}(u) q_{r}\left(u^{\prime}\right)\right) .
$$

The form of the dressing phase as an antisymmetric combination of two-magnon charges (6.31) was proposed in [34] based on physical intuition. It however remained somewhat unclear why this form applies to long-range spin chains [35]. In fact one can argue that $\theta\left(u, u^{\prime}\right)$ provides a basis for generic antisymmetric functions which vanish at $u=\infty$. This is true because $q_{r}(u)$ is a basis for alike functions of a single variable. In that sense, the form of the dressing phase is natural, because the phase is antisymmetric by construction. Nevertheless, it does not take into account that the coefficients $\beta_{r, s}$ satisfy certain perturbative bounds [24] which would not hold in an arbitrary basis for antisymmetric functions of $u, u^{\prime}$. Namely, at each order in the deformation parameter $\lambda$, only finitely many of the $\beta_{r, s}$ may be non-vanishing.

\subsection{Basis of charges $(\gamma)$}

As indicated above in (5.25), we are still free to perform a change of basis of the charge vector $\mathcal{Q}$ after the long-range deformations have been applied. These simply correspond to taking linear combinations of the long-range charges which do not affect the scattering matrix or the function $p(u)$ but modify the charge eigenvalues in an obvious way

$$
\mathcal{Q}_{r} \mapsto \gamma_{r, 0} \mathcal{N}+\gamma_{r, s} \mathcal{Q}_{s} \Longrightarrow Q_{r} \mapsto \gamma_{r, 0} N+\gamma_{r, s} Q_{s}
$$




\subsection{Higher rank and multiple magnons}

Now we wish to generalize the above results to a Lie (super) algebra $\mathfrak{g}$ of higher rank $R$. The Lie algebra has $R$ simple roots, and we shall say that they are distinguished by their flavor $a=1, \ldots, R$. The algebra is specified by a symmetric Cartan matrix $C_{a, b}$. The spins transform in a representation of the Yangian $\mathrm{Y}(\mathfrak{g})$ specified through Dynkin labels $t_{a}$ of a highest weight representation of $\mathfrak{g}^{12}$. The framework for undeformed nearest neighbor chains was developed in [36].

The generalization to higher rank consists in adding a flavor to each magnon. In a multimagnon state the number of magnons of flavor $a$ will be denoted by $M_{a}$. Their rapidities will be denoted by $u_{a, k}, k=1, \ldots, M_{a}$. A multi-magnon state is consequently denoted by

$$
\left|\left\{u_{a, k}\right\}\right\rangle \text {. }
$$

As usual, due to integrability, the multi-magnon eigenvalues $Q_{r}$ of the charges $\mathcal{Q}_{r}$ are given by sums over single-magnon eigenvalues, and similarly for the total momentum $P$

$$
P=\sum_{a=1}^{R} \sum_{k=1}^{M_{a}} p\left(t_{a}, u_{a, k}\right), \quad Q_{r}=\sum_{a=1}^{R} \sum_{k=1}^{M_{a}} q_{r}\left(t_{a}, u_{a, k}\right) .
$$

Here the spin label $t$ for the dispersion relation of a magnon of flavor $a$ is the Dynkin label $t=t_{a}$ corresponding to the same flavor $a$. This follows from the differential equation for the dispersion relations (6.10) which still holds in the case of higher rank. The value of the integration constant $t$ follows from the known result for undeformed models.

The nested Bethe ansatz ensures that scattering of magnons of two flavors is diagonal. Thus there is only one scattering factor $S_{a, b}\left(u, u^{\prime}\right)$ for each pair of magnon flavors $a, b$. The scattering factor obeys the same differential equation as before and thus the deformation takes the form

$$
S_{a, b}\left(u, u^{\prime}\right)=\exp \left(-2 \mathrm{i} \theta_{a, b}\left(u, u^{\prime}\right)\right) S_{a, b}^{\mathrm{NN}}\left(u-u^{\prime}\right) .
$$

The undeformed scattering factor for magnons of flavor $a, b$ reads [36]

$$
S_{a, b}^{\mathrm{NN}}\left(u-u^{\prime}\right)=\frac{u-u^{\prime}-\frac{\mathrm{i}}{2} C_{a, b}}{u-u^{\prime}+\frac{\mathrm{i}}{2} C_{a, b}} .
$$

The dressing phase is generated by the bilocal deformation and hence we have to use the relevant charge eigenvalues for each magnon flavor

$$
\theta_{a, b}\left(u, u^{\prime}\right)=\sum_{s>r=2}^{\infty} \beta_{r, s}\left(q_{r}\left(t_{a}, u\right) q_{s}\left(t_{b}, u^{\prime}\right)-q_{s}\left(t_{a}, u\right) q_{r}\left(t_{b}, u^{\prime}\right)\right) .
$$

\subsection{Closed chain Bethe equations}

In order to go from an infinite spin chain to a closed or open finite chain, one has to impose boundary conditions on the system. For a long-range system on a closed chain of length $N$ the periodicity condition for the multi-magnon wavefunction $\left|\left\{u_{a, k}\right\}\right\rangle$ reads

$$
\begin{aligned}
\exp \left(\mathrm{i} p\left(t_{a}, u_{a, k}\right) N\right)=\prod_{\substack{b=1 \\
(b, j) \neq(a, k)}}^{R} \prod_{a, b}\left(u_{a, k}, u_{b, j}\right) & \text { for } \quad a=1, \ldots, R \\
& \text { and } \quad k=1, \ldots, M_{a} .
\end{aligned}
$$

12 To have one Dynkin label for each flavor is merely the simplest case. In more complicated cases, e.g. tensor product representations, there can be more than one Dynkin label associated with each flavor. Also the two shifts $+\frac{i}{2} t$ and $-\frac{i}{2} t$ appearing in (6.11), (6.12) can in principle be chosen independently. 


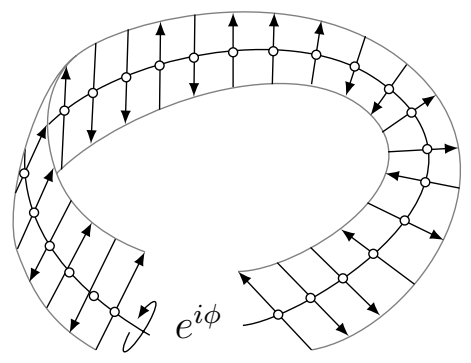

Figure 11. The long-range deformations presented in this work can also be viewed as twists of the boundary conditions.

The momentum function $p(t, u)$ and the scattering factors $S_{a, b}(u)$ have been given in the previous subsection.

Note that these Bethe equations are merely asymptotic $[18,32]$, they are valid only up to a certain perturbative order in the moduli. This is because the scattering factor is an IR quantity and the distinction between IR and UV is given by the range of the Hamiltonian. As long as the range of one of the conserved charges does not exceed the length of the chain $N$, the above asymptotic Bethe equations give the correct spectrum for this particular charge. Otherwise there can be some UV contributions that we have not taken into account in the above derivation. The range of $\mathcal{Q}_{r}$ is discussed in section 5. If one uses the one-parameter deformation $\mathcal{Q}_{r}(\lambda)=\sum_{n} \lambda^{n} \mathcal{Q}_{r}^{(n)}$ then the range of $\mathcal{Q}_{r}^{(n)}$ is $r+n$. Consequently, the Bethe ansatz gives the correct eigenvalue at order $\lambda^{N-r}$ while the order $\lambda^{N-r+1}$ is not properly defined. See also section 8.2 for a different approach, but with similar conclusions on the validity of the results.

Here we have taken the point of view that the dispersion relation and the scattering matrix have been deformed. There is another point of view that deserves being mentioned: The generating equation for our long-range integrable system (4.2) shows that the latter is obtained by a similarity transformation on a nearest neighbor model

$$
\mathcal{Q}_{r}(\xi)=\mathcal{T}(\xi) \mathcal{Q}_{r}^{\mathrm{NN}} \mathcal{T}(\xi)^{-1}
$$

It implies that the spectra of the operators must be identical. This is indeed true for infinite chains where the spectrum is continuous. On a finite system, however, the proposed deformations need not be defined consistently. For example, the boost operator (3.12) requires the definition of a spin chain origin. However, the origin is not equivalent to the site shifted by $L$ steps. In other words, the definition of the boost deformation is not compatible with closed boundary conditions. We list the various compatibilities between boundary conditions and deformations in table 1 . Whenever a boundary condition is compatible with a deformation, we can apply the above similarity transformation without deforming the spectrum. For compatible deformations the corresponding modulus will not appear in the Bethe equations. In particular, this was observed in [37] for the deformation $\mathcal{Y}_{2 r, 2 s}$ on an open chain and led to the discovery of the generating equation by reversing the argument.

The alternative point of view is that an incompatible deformation twists the boundary conditions (figure 11). Consequently, a closed long-range spin chain is nothing but a nearest neighbor model with unusual boundary conditions. For that purpose one would write the 
Table 1. Different types of deformations and their compatibility with the infinite, closed and open boundary conditions. Incompatibility corresponds to a twist of the boundary conditions. The Bethe equations remain undeformed for compatible transformations. On an open chain, deformations by bilocal operators $\mathcal{Y}_{2 r+1,2 s+1}$ composed of only odd charges violate the boundary Yang-Baxter equation [37].

\begin{tabular}{llll}
\hline $\begin{array}{l}\text { Deformation } \\
\text { generator }\end{array}$ & $\begin{array}{l}\text { Infinite } \\
\text { chain }\end{array}$ & $\begin{array}{l}\text { Closed } \\
\text { chain }\end{array}$ & $\begin{array}{l}\text { Open } \\
\text { chain }\end{array}$ \\
\hline $\mathcal{P}_{k}$ & Compatible & Incompatible & Incompatible \\
$\mathcal{Y}_{2 r+1,2 s}$ & Compatible & Incompatible & Incompatible \\
$\mathcal{Y}_{2 r+1,2 s+1}$ & Compatible & Incompatible & Not integrable \\
$\mathcal{Y}_{2 r, 2 s}$ & Compatible & Incompatible & Compatible \\
$\mathfrak{G}_{m, n}$ & Compatible & Compatible & Compatible \\
$\mathcal{L}_{l}$ & Compatible & Compatible & Compatible \\
\hline
\end{tabular}

above asymptotic Bethe equations (6.38) as the Bethe equations for a nearest neighbor chain with a twist

$$
\exp \left(\mathrm{i} p^{\mathrm{NN}}\left(t_{a}, u_{a, k}\right) N\right)=\exp \left(\mathrm{i} \phi_{a, b}\left(\left\{u_{b, j}\right\}\right)\right) \prod_{\substack{b=1 \\(b, j) \neq(a, k)}}^{R} \prod_{\substack{M_{b} \\ \text { NN }}}^{M_{a, b}}\left(u_{a, k}, u_{b, j}\right) .
$$

The twist phase depends on all Bethe roots and for the long-range system it reads

$\phi_{a, k}\left(\left\{u_{b, j}\right\}\right)=\sum_{b=1}^{R} \sum_{j=1}^{M_{b}} 2 \theta_{a, b}\left(u_{a, j}, u_{b, j}\right)-\left(p\left(t_{a}, u_{a, k}\right)-p^{\mathrm{NN}}\left(t_{a}, u_{a, k}\right)\right) N$.

Written in this fashion, the above Bethe equation is reminiscent of equations for finite-size spectra [38], see also [22].

\section{Alternating spin chains}

The construction of integrable long-range deformations presented in this paper is actually not limited to standard nearest neighbor spin chains. A possible generalization of these models is given by alternating spin chain models (cf [39]) which recently attracted notice in the context of gauge/string dualities following the work [7].

Long-range deformations. An alternating spin chain is given by a tensor product of modules transforming in alternating representations of the symmetry algebra

$$
\left|\ldots, v_{k}, \ldots, v_{k+\ell}, \ldots\right\rangle \in \cdots \otimes \mathbb{V}_{\mathrm{e}} \otimes \mathbb{V}_{\mathrm{o}} \otimes \mathbb{V}_{\mathrm{e}} \otimes \mathbb{V}_{\mathrm{o}} \otimes \cdots
$$

In fact, such a model can be brought to the form of a standard homogeneous chain by combining two adjacent modules into a larger one, $\mathbb{V}_{\mathrm{e}} \otimes \mathbb{V}_{\mathrm{o}} \rightarrow \mathbb{V}$. Consequently the nearest neighbor interactions in terms of $\mathbb{V}$ turn into next-to-nearest neighbor interactions in terms of the alternating modules $\mathbb{V}_{\mathrm{e} / \mathrm{o}}$. With regard to our work, we would like to understand the range of the deformations in the alternating chain.

Particularly interesting is the case when the symmetry algebra splits into two components $\mathfrak{g}=\mathfrak{g}_{\mathrm{e}} \oplus \mathfrak{g}_{\mathrm{o}}$. Then the even spin sites can transform in a representation of $\mathfrak{g}_{\mathrm{e}}$ while the odd 
Table 2. Long-range Hamiltonian $\mathcal{Q}_{2}$ for the $\mathfrak{g l}(K)$ spin chain at order $\lambda^{3}$ printed up to basis changes and local deformations.

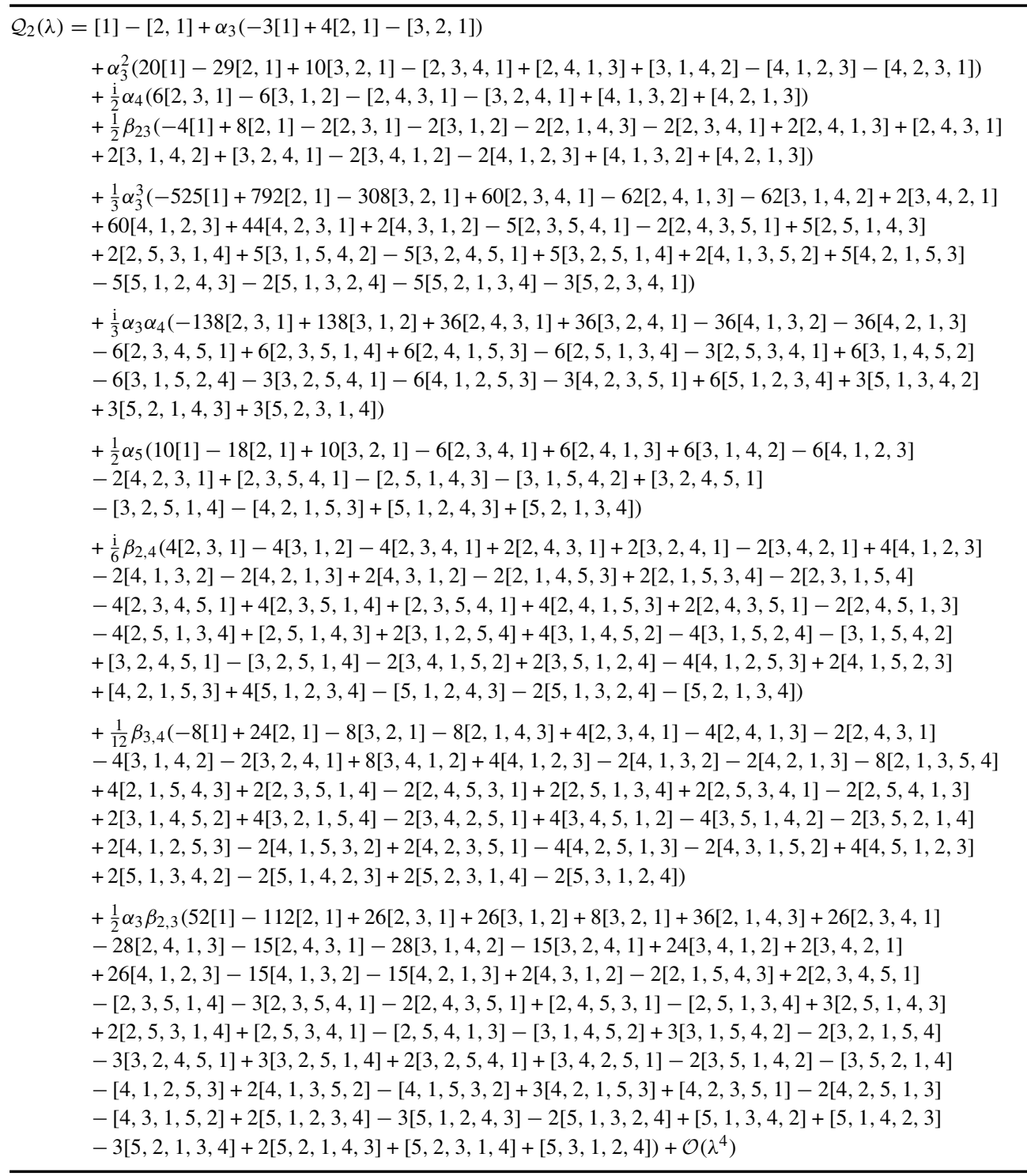

sites transform in a representation of $\mathfrak{g}_{0}$. Hence, we have two independent sets of commuting charges-one for each of the two subchains ${ }^{13}$

$$
\mathcal{Q}_{n \mathrm{e}}^{(0)}, \quad \mathcal{Q}_{n \mathrm{o}}^{(0)} .
$$

The action of $\mathcal{Q}_{n \mathrm{e}}^{(0)}$ and $\mathcal{Q}_{n \mathrm{o}}^{(0)}$ is restricted to either of the two subchains while the other subchain remains untouched. The same holds for the corresponding boosts $\mathcal{B}\left[\mathcal{Q}_{2 \mathrm{e}}^{(0)}\right]$ and $\mathcal{B}\left[\mathcal{Q}_{2 \mathrm{o}}^{(0)}\right]$. Hence, one obtains two sets $\mathcal{Q}_{n \mathrm{e}}^{(0)}, \mathcal{Q}_{\text {no }}^{(0)}$ of mutually commuting leading-order charges via (2.16).

\footnotetext{
${ }^{13}$ In principle nothing prevents us from considering more than two different alternating algebras or representations, respectively. For illustration purposes we restrict to two different symmetries here. In the general case one finds one set of conserved charges for each connected component of the symmetry algebras' Dynkin diagram.
} 
We can now use the two independent and mutually commuting sets of charges $\mathcal{Q}_{n \mathrm{e}}^{(0)}, \mathcal{Q}_{n \mathrm{o}}^{(0)}$ to construct deformations: the boosts again exclusively act on the even/odd subchain, which in turn implies that even/odd charges can only be deformed by even/odd boosts. Therefore there are two sets of boost deformation parameters $\alpha_{k \mathrm{e}}, \alpha_{k \mathrm{o}}$ that correspond to deformations by $\mathcal{B}\left[\mathcal{Q}_{k, \mathrm{e} / \mathrm{o}}\right]$. Since even/odd boosts commute with odd/even charges, i.e.

$$
\left[\mathcal{B}\left[\mathcal{Q}_{k \mathrm{x}}\right], \mathcal{Q}_{k \mathrm{y}}\right]=0, \quad \mathrm{x} \neq \mathrm{y} \in\{\mathrm{e}, \mathrm{o}\},
$$

all charge terms that are multiplied by mixed powers of the $\alpha_{k}$ coefficients, such as $\alpha_{k \mathrm{e}} \alpha_{l_{\mathrm{o}}}$ or $\alpha_{k \mathrm{e}} \alpha_{k \mathrm{o}}$, vanish. To summarize, all leading-order charges as well as all boost deformations on the two subchains completely decouple from each other. One obtains two independent sets of boost-deformed models with symmetry $\mathfrak{g}_{\mathrm{e}}$ and $\mathfrak{g}_{\mathrm{o}}$ and corresponding parameters $\alpha_{k \mathrm{e}}$ and $\alpha_{k \mathrm{o}}$. The same holds for bilocal deformations induced by operators composed of only even or only odd charges $\left[\mathcal{Q}_{r \mathrm{x}} \mid \mathcal{Q}_{s \mathrm{x}}\right], \mathrm{x} \in\{\mathrm{e}, \mathrm{o}\}$, with parameters $\beta_{r \mathrm{x}, s \mathrm{x}}$. So far the idea of an alternating spin chain is only a notational issue: two long-range chains of symmetry $\mathfrak{g}_{\mathrm{e}}$ and $\mathfrak{g}_{\mathrm{o}}$ are written as one alternating chain.

New structures appear when we introduce mixed bilocal deformations $\left[\mathcal{Q}_{r \mathrm{x}} \mid \mathcal{Q}_{s \mathrm{y}}\right], \mathrm{x} \neq$ $\mathrm{y} \in\{\mathrm{e}, \mathrm{o}\}$, corresponding to new degrees of freedom $\beta_{r \mathrm{x}, s \mathrm{y}}$. These operators deform both, the even and odd charge terms since bilocal operators that are composed of even and odd charges generally neither commute with $\mathcal{Q}_{n \text { e }}$ nor with $\mathcal{Q}_{n o}$. Therefore they result in structures that couple the two subchains. Also these new structures are fully described by the construction in the above sections.

Closed chain Bethe equations. The general Bethe equations (6.38) presented in section 6 also apply to the alternating spin chain. Considering two different alternating symmetries, the Bethe equations have to be specified to the case of a product group, i.e. to the direct sum $\mathfrak{g}_{\mathrm{e}} \oplus \mathfrak{g}_{\mathrm{o}}$. The Cartan matrix $C_{a, b}$ in (6.36) is then of block diagonal form

$$
C=\left(\begin{array}{cc}
C \mathrm{e} & 0 \\
0 & C \mathrm{o}
\end{array}\right)
$$

such that (6.38) splits into two sets of Bethe equations. These two sets are only coupled by the dressing phases $\theta_{a \mathrm{e}, b \mathrm{o}}$ and $\theta_{a \mathrm{o}, b \mathrm{e}}(6.37)$ with odd and even indices. Boost deformations do not induce a coupling of the subchains.

One gets two sets of magnons moving on either of the two chains $\left\{u_{a \mathrm{e}, k}, u_{a \mathrm{o}, k}\right\}$. Since the odd integrable charges do not see magnons on the even spin chain and vice versa,

$$
Q_{n \mathrm{x}}=\sum_{a=1}^{R \mathrm{x}} \sum_{k=1}^{M_{a \mathrm{x}}} q_{n \mathrm{x}}\left(t_{a \mathrm{x}}, u_{a \mathrm{x}, k}\right), \quad \mathrm{x} \in\{\mathrm{e}, \mathrm{o}\}
$$

we have to distinguish two sets of one-magnon charge eigenvalues

$q_{n \mathrm{x}}(t, u)=\frac{\mathrm{i}}{n-1}\left(\frac{1}{x_{\mathrm{x}}\left(u+\frac{\mathrm{i}}{2} t\right)^{n-1}}-\frac{1}{x_{\mathrm{x}}\left(u-\frac{\mathrm{i}}{2} t\right)^{n-1}}\right), \quad \mathrm{x} \in\{\mathrm{e}, \mathrm{o}\}$.

Here, the rapidity map $x_{\mathrm{e} / \mathrm{o}}(u)$ is parametrized by the moduli $\alpha_{k \mathrm{e}}$ or $\alpha_{k_{\mathrm{o}}}$, respectively.

As an example consider the alternating spin chain with spins transforming in the fundamental and anti-fundamental representation of $\mathfrak{s u}(2)$, i.e. $\mathfrak{g}_{\mathrm{e}} \oplus \mathfrak{g}_{\mathrm{o}}=\mathfrak{s u}(2) \oplus \mathfrak{s u}(2)$ and

$$
\left(\begin{array}{ll}
C_{\mathrm{e}, \mathrm{e}} & C_{\mathrm{e}, \mathrm{o}} \\
C_{\mathrm{o}, \mathrm{e}} & C_{\mathrm{o}, \mathrm{o}}
\end{array}\right)=\left(\begin{array}{ll}
2 & 0 \\
0 & 2
\end{array}\right)
$$



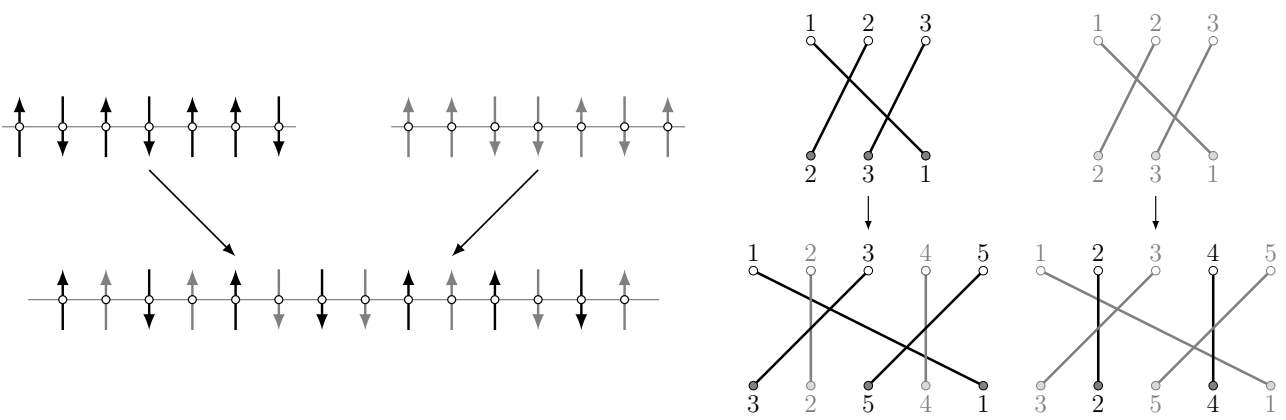

Figure 12. The alternating $\mathfrak{g l}\left(K_{\mathrm{e}}\right) \oplus \mathfrak{g l}\left(K_{\mathrm{o}}\right)$ spin chain is given by two staggered versions of the standard $\mathfrak{g l}(K)$ spin chain. We can use the same operator notation as before if we insert identity legs at every second position of the permutation operators and let them act on even or odd positions of the chain only.

The Bethe equations are given by

$$
\begin{aligned}
& \frac{x_{\mathrm{e}}\left(u_{\mathrm{e}, k}+\frac{\mathrm{i}}{2}\right)^{N}}{x_{\mathrm{e}}\left(u_{\mathrm{e}, k}-\frac{\mathrm{i}}{2}\right)^{N}}=\prod_{\substack{j=1 \\
j \neq k}}^{M_{\mathrm{e}}} \frac{u_{\mathrm{e}, k}-u_{\mathrm{e}, j}+\mathrm{i}}{u_{\mathrm{e}, k}-u_{\mathrm{e}, j}-\mathrm{i}} \exp \left(2 \mathrm{i} \theta_{\mathrm{e}, \mathrm{e}}\left(u_{\mathrm{e}, k}, u_{\mathrm{e}, j}\right) \prod_{\ell=1}^{M_{\mathrm{o}}} \exp \left(2 \mathrm{i} \theta_{\mathrm{e}, \mathrm{o}}\left(u_{\mathrm{e}, k}, u_{\mathrm{o}, \ell}\right)\right),\right. \\
& \frac{x_{\mathrm{o}}\left(u_{\mathrm{o}, k}+\frac{\mathrm{i}}{2}\right)^{N}}{x_{\mathrm{o}}\left(u_{\mathrm{o}, k}-\frac{\mathrm{i}}{2}\right)^{N}}=\prod_{\substack{j=1 \\
j \neq k}}^{M_{\mathrm{o}}} \frac{u_{\mathrm{o}, k}-u_{\mathrm{o}, j}+\mathrm{i}}{u_{\mathrm{o}, k}-u_{\mathrm{o}, j}-\mathrm{i}} \exp \left(2 \mathrm{i} \theta_{\mathrm{o}, \mathrm{o}}\left(u_{\mathrm{o}, k}, u_{\mathrm{o}, j}\right)\right) \prod_{\ell=1}^{M_{\mathrm{e}}} \exp \left(2 \mathrm{i} \theta_{\mathrm{o}, \mathrm{e}}\left(u_{\mathrm{o}, k}, u_{\mathrm{e}, \ell}\right)\right) .
\end{aligned}
$$

The mixed dressing phase takes the form

$\theta_{\mathrm{x}, \mathrm{y}}\left(u, u^{\prime}\right)=-\theta_{\mathrm{y}, \mathrm{x}}\left(u^{\prime}, u\right)=\sum_{s, r=2}^{\infty} \beta_{r \mathrm{x}, s \mathrm{y}} q_{r \mathrm{x}}(u) q_{s \mathrm{y}}\left(u^{\prime}\right), \quad \mathrm{x} \neq \mathrm{y} \in\{\mathrm{e}, \mathrm{o}\}$.

The two sets of Bethe equations are only coupled by this phase.

The $\mathfrak{g l}\left(K_{\mathrm{e}}\right) \oplus \mathfrak{g l}\left(K_{\mathrm{o}}\right)$ Alternating Spin Chain. In order to explicitly study the $\mathfrak{g l}(K \mathrm{e}) \oplus \mathfrak{g l}(K \mathrm{o})$ chain (cf. figure 12), we introduce two new types of permutation symbols whose first leg acts on even/odd spin chain sites only

$$
\mathcal{L}_{\mathrm{e}}=[\pi(1), \pi(2), \ldots, \pi(n)]_{\mathrm{e}}, \quad \mathcal{L}_{\mathrm{o}}=[\pi(1), \pi(2), \ldots, \pi(m)]_{\mathrm{o}} .
$$

The action of $[3,2,1]_{\mathrm{e}}$ for instance is given by

$$
\begin{gathered}
{[3,2,1]_{\mathrm{e}}|\ldots, \stackrel{e}{1}, \stackrel{\mathrm{o}}{2}, \stackrel{\mathrm{e}}{3}, \stackrel{\mathrm{o}}{4}, \stackrel{\mathrm{e}}{5}, \ldots\rangle=\cdots+|\ldots, \stackrel{\mathrm{e}}{3}, \stackrel{\mathrm{o}}{2}, \stackrel{\mathrm{e}}{1}, \stackrel{\mathrm{o}}{4}, \stackrel{\mathrm{e}}{5}, \ldots\rangle} \\
+|\ldots, \stackrel{\mathrm{e}}{1}, \stackrel{\mathrm{o}}{2}, \stackrel{\mathrm{e}}{5}, \stackrel{\mathrm{o}}{4}, \stackrel{\mathrm{e}}{3}, \ldots\rangle+\cdots
\end{gathered}
$$

The alternating $\mathfrak{g l}\left(K_{\mathrm{e}}\right) \oplus \mathfrak{g l}\left(K_{\mathrm{o}}\right)$ model has two operators of minimum range 3 being the standard $\mathfrak{g l}(K)$ Hamiltonian on the staggered subchains

$$
\mathcal{Q}_{2 \mathrm{e}}^{(0)}=[1]_{\mathrm{e}}-[3,2,1]_{\mathrm{e}}, \quad \mathcal{Q}_{2 \mathrm{o}}^{(0)}=[1]_{\mathrm{o}}-[3,2,1]_{\mathrm{o}} .
$$

Here we have simply taken the leading order $\mathfrak{g l}(K)$ Hamiltonian (2.16), stretched it by a central identity leg and restricted the action of the permutation symbol to even or odd sites, respectively. For better readability we denote the part acting on the odd subchain in gray. Both copies of the resulting Hamiltonian commute with each other and thus describe a system of two staggered nearest neighbor spin chains. The form of the Hamiltonian implies that also all 
leading-order charges generated through (2.14) have such an alternating structure with identity legs on every other permutation site, e.g.

$$
\begin{aligned}
& \mathcal{Q}_{3 \mathrm{e}}^{(0)}=\frac{\mathrm{i}}{2}\left([5,2,1,4,3]_{\mathrm{e}}-[3,2,5,4,1]_{\mathrm{e}}\right), \\
& \mathcal{Q}_{3 \mathrm{o}}^{(0)}=\frac{\mathrm{i}}{2}\left([5,2,1,4,3]_{\mathrm{o}}-[3,2,5,4,1]_{\mathrm{o}}\right) .
\end{aligned}
$$

As discussed at the beginning of this section, this is due to the fact that two local operators of this staggered form (including boosts) acting on either even or odd sites only, commute with each other, e.g.

$\left[[5,2,1,4,3]_{\mathrm{e}},[7,2,3,4,5,6,1]_{\mathrm{o}}\right]=0, \quad\left[\mathcal{B}\left[[3,2,1]_{\mathrm{o}}\right],[3,2,5,4,1]_{\mathrm{e}}\right]=0$.

Considering bilocal operators, we find the new interaction terms described in the previous paragraphs. These terms do not only act on one of the two staggered spin chains but on both chains at the same time. Thus we obtain terms like

$$
\begin{aligned}
& \mathcal{Q}_{2 \mathrm{e}}=\mathcal{Q}_{2 \mathrm{e}}^{(0)}+\beta_{2 \mathrm{e}, 3 \mathrm{o}} \mathcal{Q}_{2 \mathrm{e}}^{[2 \mathrm{e} \mid 3 \mathrm{o}]}+\cdots, \quad \text { with } \\
& \begin{aligned}
\mathcal{Q}_{2 \mathrm{e}}^{[2 \mathrm{e} \mid 3 \mathrm{o}]}=2(- & {[3,4,5,6,1,2]_{\mathrm{e}}+[3,6,5,2,1,4]_{\mathrm{e}}+[5,4,1,6,3,2]_{\mathrm{e}}-[5,6,1,2,3,4]_{\mathrm{e}} } \\
& \quad-[3,4,5,6,1,2]_{\mathrm{o}}+[3,6,5,2,1,4]_{\mathrm{o}}+[5,4,1,6,3,2]_{\mathrm{o}}-[5,6,1,2,3,4]_{\mathrm{o}},
\end{aligned}
\end{aligned}
$$

which act non-trivially on both chains. Note, though, that even and odd numbers are always on even and odd positions of the permutation symbols, respectively. That is, also these interactions do not interchange sites of the two staggered chains. Similarly, one finds new structures proportional to mixed $\alpha \beta$ coefficients like for instance $\alpha_{3 \mathrm{e}} \beta_{2 \mathrm{o}, 3 \mathrm{e}}$.

Interaction range for $\mathfrak{g l}\left(K_{\mathrm{e}}\right) \oplus \mathfrak{g l}\left(K_{\mathrm{o}}\right)$. All charge terms that emerge from deformations by boost or bilocal operators acting on either the even or the odd chain exclusively are given by stretched versions of the corresponding $\mathfrak{g l}(K)$ terms. Their interaction range is thus given by (cf (5.24))

$$
\left|\mathcal{Q}_{n \mathrm{x}}^{\left[k_{1} \mathrm{x}, \ldots, k_{t} \mathrm{x}, r_{1} \mathrm{x}\left|s_{1} \mathrm{x}, \ldots, r_{u} \mathrm{x}\right| s_{u} \mathrm{x}\right]}\right|=2\left(n+\sum_{\ell=1}^{t}\left(k_{\ell}-2\right)+\sum_{\ell=1}^{u}\left(s_{\ell}-1\right)\right)-1, \quad \mathrm{x} \in\{\mathrm{e}, \mathrm{o}\} .
$$

At leading order, the new interaction terms arising from deformations by bilocal operators composed of one even and one odd charge apparently have interaction range

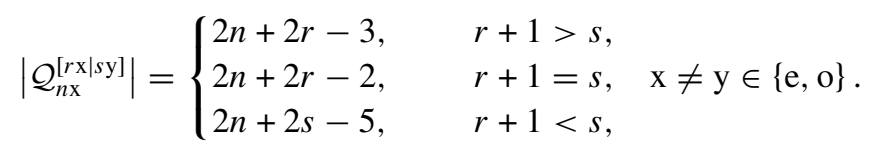

Number of crossings for $\mathfrak{g l}(2) \oplus \mathfrak{g l}(2)$. Again, a particularly interesting case is the $\mathfrak{g l}(2) \oplus \mathfrak{g l}(2)$ chain since it incorporates the $\mathfrak{s u}(2) \oplus \mathfrak{s u}(2)$ sector spin chain of $\mathcal{N}=6$ superconformal ChernSimons theory. The natural building block (cf the Hamiltonian (7.12)) corresponding to the term 'crossing' for the alternating $\mathfrak{g l}(2)$ spin chain is given by the diagrams

$$
\Varangle \text { and } \nVdash
$$

We take a look at the $\mathfrak{g l}\left(K_{\mathrm{e}}\right) \oplus \mathfrak{g l}\left(K_{\mathrm{o}}\right)$ Hamiltonian printed in table 3 at the end of this paper: the first interesting operator is given by the permutations multiplied by $\alpha_{3 \mathrm{x}}, \mathrm{x} \in\{\mathrm{e}, \mathrm{o}\}$. The 
Table 3. Long-range Hamiltonian for the alternating spin chain printed up to basis changes and local deformations. The first part gives two stretched copies of the $\mathfrak{g l}(K)$ Hamiltonian (table 2) acting on odd and even spin sites, respectively. The second part contains novel interaction symbols which act non-trivially on the odd and even copy of the chain at the same time. The Hamiltonian of the integrable spin chain supposedly describing the $\mathfrak{s u}(2) \times \mathfrak{s u}(2)$ sector of $\mathcal{N}=6$ superconformal Chern-Simons theory is given by a specific choice of the free parameters.

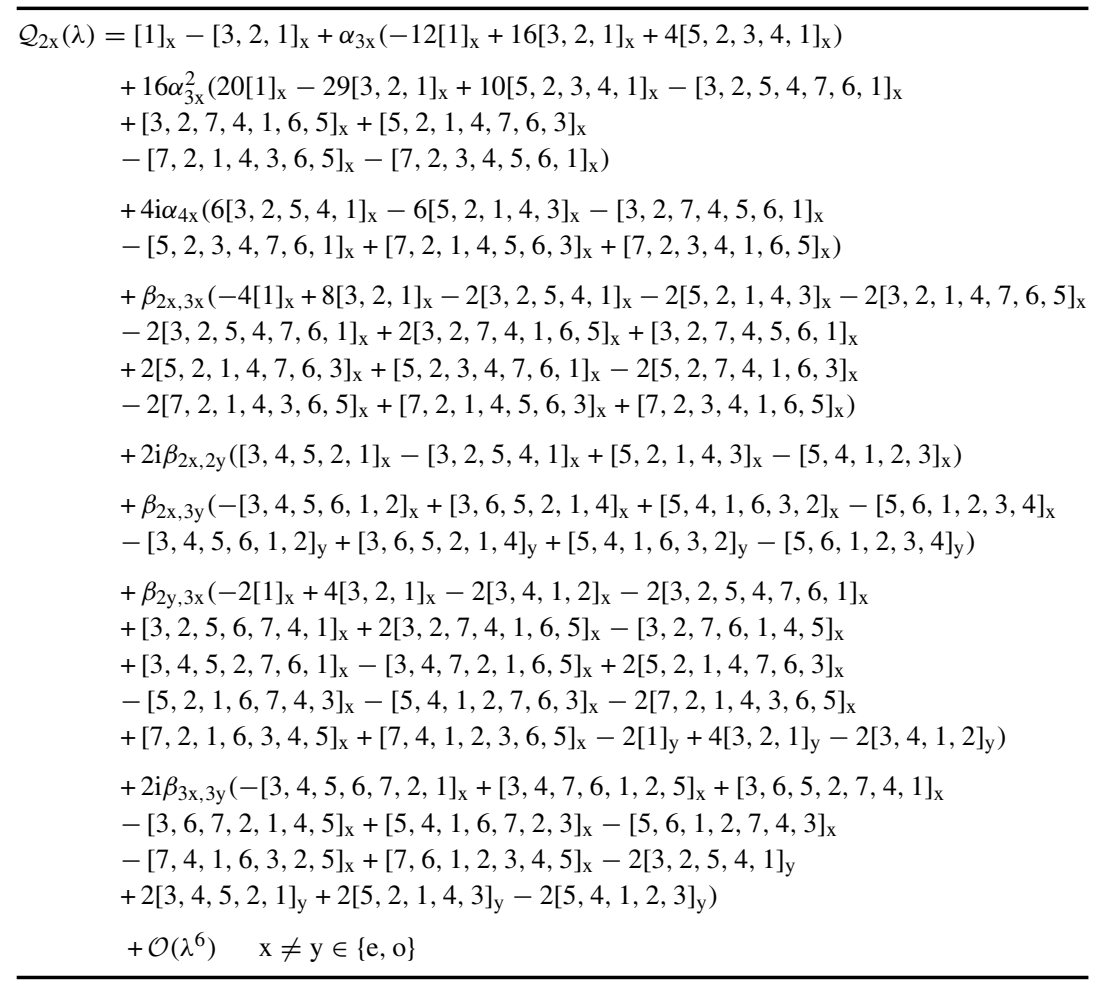

term with the highest number of elementary permutations is

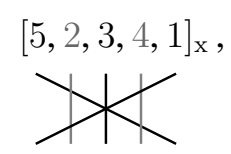

which naively contains seven elementary permutations. This permutation symbol, however, can be considered as the combination of the two operators

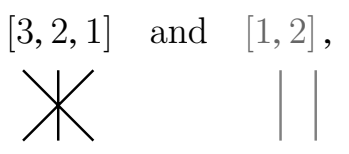

acting on the two staggered spin chains simultaneously. As for the standard $\mathfrak{g l}(2)$ chain we can use the $\varepsilon_{i j k}$-identity (5.38) for each of the alternating $\mathfrak{g l}(2)$ symmetries to reduce the number of crossings

$$
\begin{aligned}
& {[5,2,3,4,1]_{\mathrm{x}}=[3,2,5,4,1]_{\mathrm{x}}+[5,2,1,4,3]_{\mathrm{x}}-2[3,2,1]_{\mathrm{x}}+[1]_{\mathrm{x}} .} \\
& Y K=Y K+Y X+1
\end{aligned}
$$


In particular, we now have expressed the permutation symbol (7.19) in terms of the elementary crossings (7.18). Similarly, it appears to be possible to write the higher $\alpha_{k x}$ as well as the $\beta_{r \mathrm{x}, s \mathrm{x}}$ structures in terms of elementary crossings without additional assumptions.

The new terms corresponding to deformations with mixed bilocal operators $\left[\mathcal{Q}_{r \mathrm{x}} \mid \mathcal{Q}_{s \mathrm{y}}\right]$ composed of charges acting on the odd and even chain are given by the permutation operators multiplied by $\beta_{r \mathrm{x}, s \mathrm{y}}$ (cf table 3 ). An example is given by the following operator proportional to $\beta_{2 x, 3 y}$ :

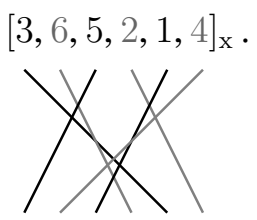

This structure can be considered as the combination of

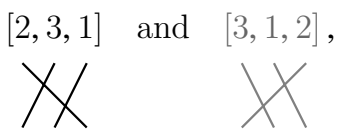

such that using the identity

$$
Y=||
$$

we can again explicitly rewrite the diagram in terms of the six-vertices (7.18)

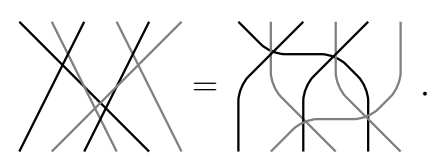

Our experiments with the $\mathfrak{g l}\left(K_{\mathrm{e}}\right) \oplus \mathfrak{g l}\left(K_{\mathrm{o}}\right)$ Hamiltonian in table 3 therefore suggest a formula for the number of crossings in analogy to (5.39)

$\left|\mathcal{Q}_{t \mathrm{x}}^{\left[k_{1}, \ldots, k_{t}, r_{1}\left|s_{1}, \ldots, r_{u}\right| s_{u}\right]}\right|_{\mathfrak{g l}(2) \oplus \mathfrak{g l}(2)}=t-1+\sum_{\ell=1}^{u}\left(r_{\ell}+s_{\ell}-2\right)+\sum_{\ell=1}^{t}\left(k_{\ell}-2\right)$.

Parameter restrictions from the $\mathfrak{s u}(2) \oplus \mathfrak{s u}(2)$ sector of $\mathcal{N}=6$ superconformal Chern-Simons theory. Considering restrictions from gauge theory, the main difference to the $\mathcal{N}=4$ case is the scalar interaction $\sim \lambda^{2} Y^{A} Y_{A}^{\dagger} Y^{B} Y_{B}^{\dagger} Y^{C} Y_{C}^{\dagger}$ in the $\mathcal{N}=6$ Lagrangian (cf [7]). Disregarding other interactions, this translates to the vertex correspondence

$$
\text { \or } ¥ \sim \lambda^{2}
$$

Accordingly, only even powers of the coupling $\lambda$ contribute to the integrable charges and the spin chain and gauge theory Hamiltonian are related by

$$
\mathcal{H}\left(\lambda^{2}\right)=\mathcal{H}^{(0)}+\lambda^{2} \mathcal{Q}_{2 \mathrm{e}}\left(\lambda^{2}\right)+\lambda^{2} \mathcal{Q}_{2 \mathrm{e}}\left(\lambda^{2}\right) .
$$


We thus find the same gauge theory restrictions on the parameter functions as for the $\mathcal{N}=4$ chain

$$
\begin{aligned}
& \alpha_{k \mathrm{x}}\left(\lambda^{2}\right)=\mathcal{O}\left(\lambda^{2(k-2)}\right), \\
& \beta_{r \mathrm{x}, s \mathrm{y}}\left(\lambda^{2}\right)=\mathcal{O}\left(\lambda^{2(r+s-2)}\right), \quad \mathrm{x}, \mathrm{y} \in\{\mathrm{e}, \mathrm{o}\} .
\end{aligned}
$$

Similar to the $\mathcal{N}=4$ case, we believe these restrictions to hold also if one includes non-scalar interactions from the $\mathcal{N}=6$ Lagrangian.

The staggered $\mathfrak{g l}\left(K_{\mathrm{e}}\right) \oplus \mathfrak{g l}\left(K_{\mathrm{o}}\right)$ Hamiltonian including all deformation degrees of freedom is printed at the end of this paper up to order $\mathcal{O}\left(\lambda^{6}\right)$.

\section{Inhomogeneous versus long-range spin chains}

All of the above considerations were based on the assumption of infinite chains. Even the Bethe ansatz for the spectrum of closed finite chains in section 6 retains the notion of infinite extent: it is asymptotic and valid only as long as the range of the interactions does not exceed the length of the chain, that is only to finite order in the deformations [18, 32] (see also [6, 40] for explicit considerations of the failure beyond this order).

In the following we reconsider a relation of long range to inhomogeneous integrable spin chains without dressing phase, i.e. in the absence of bilocal deformations, cf [6]

$$
\beta_{r, s}=0 .
$$

It allows us to formulate consistently a large class of integrable models on finite chains. Unfortunately, the problem of undefined wrapping interactions enters in this class of models as well.

For presentation purposes, we will only consider the simplest example of spins transforming in the fundamental representation of $\mathfrak{s u}(2)$, a Heisenberg $\mathrm{XXX}_{1 / 2}$ chain. This is sufficient because only the momentum-carrying Bethe roots of the first level will be important; the auxiliary levels of the Bethe ansatz for a higher rank symmetry algebra are manifestly identical on both sides of the relation.

\subsection{Bethe equations}

The standard Bethe equations of a closed Heisenberg $\mathrm{XXX}_{1 / 2}$ chain with inhomogeneities are given by

$$
\frac{P_{N}\left(u_{k}+\frac{\mathrm{i}}{2}\right)}{P_{N}\left(u_{k}-\frac{\mathrm{i}}{2}\right)}=\prod_{\substack{j=1 \\ j \neq k}}^{M} \frac{u_{k}-u_{j}+\mathrm{i}}{u_{k}-u_{j}-\mathrm{i}}, \quad P_{N}(u)=\prod_{k=1}^{N}\left(u-\mu_{k}\right) .
$$

Here $P_{N}$ is a polynomial of degree $N$ incorporating the inhomogeneities $\mu_{k}$. A homogeneous chain is obtained by setting all parameters $\mu_{k}$ to be the same, conventionally $\mu_{k}=0$. The above equations are reminiscent of the BDS equations [6] for a closed long-range $\mathrm{XXX}_{1 / 2}$ chain without bilocal deformations, $\beta_{r, s}=0$,

$$
\frac{x\left(u_{k}+\frac{\mathrm{i}}{2}\right)^{N}}{x\left(u_{k}-\frac{\mathrm{i}}{2}\right)^{N}}=\prod_{\substack{j=1 \\ j \neq k}}^{M} \frac{u_{k}-u_{j}+\mathrm{i}}{u_{k}-u_{j}-\mathrm{i}}, \quad x(u)=u-\frac{\alpha_{3}}{u}+\cdots
$$

The boost parameters $\alpha_{k}$, however, are chosen generically

$$
u=x+\sum_{k=3}^{\infty} \frac{\alpha_{k}}{u^{k-2}}, \quad \alpha_{k}=\mathcal{O}\left(\lambda^{k-2}\right) .
$$


Now it has been observed that remarkably $x(u)^{N}$ is a polynomial of degree $N$ in $u$ up to terms of order $\mathcal{O}\left(\lambda^{N}\right)[6]$

$$
P_{N}(u)=x(u)^{N}+\mathcal{O}\left(\lambda^{N}\right) .
$$

There is a simple proof for this observation: it is obvious from (8.3) that the expansion of $x(u)^{N}$ in powers of $\lambda$ contains only terms $u^{k}$ with $k \leqslant N$. One then needs to show that all the terms with $k<0$ vanish up to $\mathcal{O}\left(\lambda^{N}\right)$. This is equivalent to showing

$$
\oint_{u=0} x(u)^{N} f(u) \mathrm{d} u=\mathcal{O}\left(\lambda^{N}\right)
$$

for all functions $f(u)$ which are analytic at $u=0$. We perform a change of variable to $x$

$$
\oint_{x \approx 0} x^{N} f(u(x)) u^{\prime}(x) \mathrm{d} x \stackrel{?}{=} \mathcal{O}\left(\lambda^{N}\right) .
$$

We note that the transformation (8.4) relates the point $u=0$ to two points $x=\mathcal{O}\left(\lambda^{1 / 2}\right)$ as well as several points $x=\mathcal{O}(\lambda)$ which all lie near $x=0$. We can thus expand the integrand around $x=0$ and pick out the residue at $x=0$. The transformation (8.4) states that $u(x)$ consists of terms $\lambda^{k} / x^{k}$ and $u^{\prime}(x)$ of terms $\lambda^{k} / x^{k+1}$. Altogether the term $x^{N-N-1}$ in the integrand must be of order $\lambda^{N}$ which proves the above statement (8.5).

The Bethe equations for the long-range chain are asymptotic, they yield results which are valid up to terms of at most $\mathcal{O}\left(\lambda^{N}\right)$. Therefore it is safe to replace $x(u)^{N}$ by a suitable $P_{N}(u)$ in (8.3) and obtain exactly the inhomogeneous Bethe equations (8.2). The latter are completely well-defined equations even for finite chains. For example it is safe to assume that they are complete, i.e. they have precisely $2^{N}$ physical solutions reproducing the dimension of the Hilbert space of a Heisenberg chain of length $N$. Consequently the map appears to open a window to finite-size corrections within long-range chains.

\subsection{Charge eigenvalues}

For a solution of the above inhomogeneous Bethe equations, the eigenvalues of the standard transfer matrix are given by the relation

$$
T(u)=P_{N}\left(u+\frac{\mathrm{i}}{2}\right) \prod_{k=1}^{M} \frac{u-u_{k}-\mathrm{i}}{u-u_{k}}+P_{N}\left(u-\frac{\mathrm{i}}{2}\right) \prod_{k=1}^{M} \frac{u-u_{k}+\mathrm{i}}{u-u_{k}} .
$$

Alternatively this equation can be viewed as a Baxter equation: absence of poles at $u=u_{k}$ is equivalent to the Bethe equations. Consequently $T(u)$ is a polynomial of degree $N$ encoding the $N$ independent charge eigenvalues for this state.

As already seen, the eigenvalues of the long-range charges take the form

$Q_{r}=\sum_{k=1}^{M} q_{r}\left(u_{k}\right)+\mathcal{O}\left(\lambda^{N+1-r}\right), \quad q_{r}(u)=\frac{\mathrm{i}}{r-1}\left(\frac{1}{x\left(u+\frac{\mathrm{i}}{2}\right)^{r-1}}-\frac{1}{x\left(u-\frac{\mathrm{i}}{2}\right)^{r-1}}\right)$.

Unfortunately, these charges make explicit reference to the magnons whereas in standard integrable spin chains all observable charges follow directly from transfer matrix eigenvalues $T(u)$. This would also be preferable from the analytic Baxter-type equation point of view. Indeed we find a way to extract the above $Q_{r}$ directly from $T(u)$ by the following residue integral:

$Q_{r}=\mp \frac{1}{2 \pi \mathrm{i}} \oint_{u= \pm \mathrm{i} / 2} \frac{\mathrm{i}}{(r-1) x\left(u \mp \frac{\mathrm{i}}{2}\right)^{r-1}} \mathrm{~d} \log \frac{T(u)}{P_{N}\left(u \pm \frac{\mathrm{i}}{2}\right)}+\mathcal{O}\left(\lambda^{N+1-r}\right)$. 
Here the different signs \pm indicate two possible choices yielding the same result. The contour of the integral is meant to encircle the small branch cut of $x\left(u \mp \frac{\mathrm{i}}{2}\right)=u \mp \frac{\mathrm{i}}{2}+\mathcal{O}(\lambda)$ near $u= \pm \frac{\mathrm{i}}{2}$. Our curious observation is that (8.10) holds up to wrapping order in $\lambda$.

The equality can be proven as follows: there are two terms in $T\left(u+\frac{\mathrm{i}}{2}\right) / P_{N}(u+\mathrm{i})$, cf (8.8). If there was only the first term,

$$
Q_{r}=-\frac{1}{2 \pi \mathrm{i}} \oint_{u=0} \frac{\mathrm{i}}{(r-1) x(u)^{r-1}} \mathrm{~d} \log \prod_{k=1}^{M} \frac{u-u_{k}-\frac{\mathrm{i}}{2}}{u-u_{k}+\frac{\mathrm{i}}{2}}
$$

the equality of (8.9) and (8.10) follows simply by inverting the integration contour and summing over the poles at $u=u_{k} \pm \frac{\mathrm{i}}{2}$. The remainder thus takes the form

$$
\Delta Q_{r}=-\frac{1}{2 \pi} \oint_{u=0} \frac{1}{(r-1) x(u)^{r-1}} \mathrm{~d} \log \left(1+\frac{P_{N}(u)}{P_{N}(u+\mathrm{i})} \prod_{k=1}^{M} \frac{u-u_{k}+\frac{3 \mathrm{i}}{2}}{u-u_{k}-\frac{\mathrm{i}}{2}}\right) .
$$

To the order we are working at we can substitute $P_{N}(u)=x(u)^{N}$ which allows us to write

$$
\begin{aligned}
\Delta Q_{r} & =-\frac{1}{2 \pi} \oint_{u=0} \frac{\mathrm{d} \log \left(1+x(u)^{N} F(u)\right)}{(r-1) x(u)^{r-1}} \\
& =-\frac{1}{2 \pi} \oint_{u=0} \frac{\mathrm{d}\left(x(u)^{N} F(u)\right)}{(r-1) x(u)^{r-1}\left(1+x(u)^{N} F(u)\right)} .
\end{aligned}
$$

Here $F(u)$ is some function analytic at $u=0$, a fact we can use to simplify further

$$
\begin{aligned}
\Delta Q_{r} & =-\frac{1}{2 \pi} \oint_{u=0} \mathrm{~d} u F(u) x(u)^{N-r} x^{\prime}(u) \\
& =\frac{1}{2 \pi} \oint_{u=0} \frac{x(u)^{N+1-r}}{N+1-r} \mathrm{~d} F(u)=\mathcal{O}\left(\lambda^{N+1-r}\right) .
\end{aligned}
$$

The point is that $x^{N+1-r}(u)$ is a polynomial (8.5) up to terms of order $\mathcal{O}\left(\lambda^{N+1-r}\right)$ and hence the residue integral must be trivial at this order.

\subsection{Charge operators}

Above we have seen that the charge eigenvalues $Q_{r}$ for a long-range chain can be obtained from the transfer matrix eigenvalues of an inhomogeneous chain. This statement on the spectra of commuting operators can be lifted to a statement of the operators themselves: up to a similarity transformation, the long-range charges $\mathcal{Q}_{r}$ must equal

$$
\mathcal{Q}_{r} \simeq-\frac{1}{2 \pi \mathrm{i}} \oint_{u=0} \frac{\mathrm{i}}{(r-1) x(u)^{r-1}} \mathrm{~d} \log \frac{\mathcal{T}\left(u+\frac{\mathrm{i}}{2}\right)}{P_{N}(u+\mathrm{i})}+\mathcal{O}\left(\lambda^{N+1-r}\right) .
$$

As an example we now want to see explicitly how to obtain the first long-range deformation of the Hamiltonian $\mathcal{Q}_{2}$ from an inhomogeneous spin chain. In particular, we will have to find a suitable similarity transformation.

We first expand the transfer matrix according to the usual relation into a sequence of charges

$$
\frac{\mathcal{T}\left(u+\frac{\mathrm{i}}{2}\right)}{P_{N}(u+\mathrm{i})}=\mathcal{U} \exp \left(\sum_{r=2}^{\infty} \mathrm{i} u^{r-1} \overline{\mathcal{Q}}_{r}\right) .
$$

Here $\mathcal{U}$ denotes the operator that shifts the sites of the spin chain by one unit. The above relation determines the long-range Hamiltonian $\mathcal{Q}_{2}$ in terms of the inhomogeneous charges $\overline{\mathcal{Q}}_{r}$

$$
\mathcal{Q}_{2} \simeq \overline{\mathcal{Q}}_{2}+3 \lambda \overline{\mathcal{Q}}_{4}+\mathcal{O}\left(\lambda^{2}\right)
$$


This is in fact precisely the same relation as derived in section 5.2. The transfer matrix $\mathcal{T}$ is defined as the trace of a product of $R$-matrices (see [41] for a review of the $R$-matrix formalism)

$$
\mathcal{T}\left(u+\frac{\mathrm{i}}{2}\right)=\operatorname{Tr}_{0} \mathcal{R}_{0,1}\left(u-\mu_{1}\right) \mathcal{R}_{0,2}\left(u-\mu_{2}\right) \cdots \mathcal{R}_{0, N}\left(u-\mu_{N}\right) .
$$

Up to an overall factor the $\mathfrak{s u}(2) R$-matrix ${ }^{14}$ in the fundamental representation takes the form

$$
\mathcal{R}_{k, l}(u)=u \mathcal{I}_{k, l}+\mathrm{i} \mathcal{P}_{k, l},
$$

where $\mathcal{I}_{k, l}$ is the identity operator acting on the spin sites $k$ and $l$, while $\mathcal{P}_{k, l}$ exchanges the two spins. The resulting inhomogeneous Hamiltonian $\overline{\mathcal{Q}}_{2}$ is given by

$$
\begin{aligned}
\overline{\mathcal{Q}}_{2}=\sum_{k=1}^{N}\left([1]_{k}\right. & \left.-[2,1]_{k}\right)+\mathrm{i} \sum_{k=1}^{N} \mu_{k}\left([3,1,2]_{k-1}-[2,3,1]_{k-1}\right) \\
& +\sum_{k=1}^{N} \mu_{k}^{2}\left(-[1]_{k}+2[2,1]_{k}-[3,2,1]_{k-1}\right) \\
& +\sum_{k=1}^{N} \mu_{k-1} \mu_{k}\left([2,3,4,1]_{k-2}-[2,4,1,3]_{k-2}+[4,1,2,3]_{k-2}+[3,1,4,2]_{k-2}\right) \\
& +\mathcal{O}\left(\lambda^{3}\right) .
\end{aligned}
$$

Note that the inhomogeneities $\mu_{k}$ are small and thus to leading order the charges $\overline{\mathcal{Q}}_{r}$ coincide with the charges $\mathcal{Q}_{r}^{\mathrm{NN}}$ of a nearest neighbor chain; only the higher orders in $\lambda$ contain inhomogeneous terms.

We now have to find a similarity transformation that turns the inhomogeneous terms into homogeneous long-range interactions

$$
\mathcal{Q}_{2}=\mathcal{S}\left(\overline{\mathcal{Q}}_{2}+3 \lambda \overline{\mathcal{Q}}_{4}+\cdots\right) \mathcal{S}^{-1}
$$

An ansatz for the similarity transformation $\mathcal{S}$ relating the two spin chain Hamiltonians is

$\mathcal{S}=\exp \left(\mathrm{i} \sum_{k=1}^{N} v_{k}\left([1]_{k}-[2,1]_{k}\right)-\sum_{k=1}^{N} \rho_{k}\left([3,1,2]_{k-1}-[2,3,1]_{k-1}\right)+\cdots\right)$

In order to find suitable coefficients $v_{k}$ and $\rho_{k}$ we have to be more specific about the inhomogeneities $\mu_{k}$. The factorization of the long-range polynomial $P_{N}(u)$ in (8.2) yields the following set of inhomogeneities [6]:

$$
\mu_{k}=2 \sqrt{\alpha_{3}} \cos \frac{\pi(2 k-1)}{2 N}+\frac{\alpha_{4}}{\alpha_{3}} \cos \frac{\pi(2 k-1)}{N}+\cdots
$$

Note that the order of the inhomogeneities matters only to a certain extent; different orderings are related by similarity transformations. We choose the natural ordering for which the similarity transformation to the long-range model is the simplest. The parameters $v_{k}$ and $\rho_{k}$ then have to be chosen as

$$
v_{k}=\sqrt{\alpha_{3}} \frac{\sin \frac{\pi k}{N}}{\sin \frac{\pi}{2 N}}+\frac{\alpha_{4}}{\alpha_{3}} \frac{\sin \frac{2 \pi k}{N}}{2 \sin \frac{\pi}{N}}+\cdots, \quad \rho_{k}=\alpha_{3} \frac{\sin \frac{\pi(2 k-1)}{N}}{2 \sin \frac{\pi}{2 N}}+\cdots .
$$

This transformation (8.21), (8.22) cancels the inhomogeneous $\sqrt{\alpha_{3}}$ term as well as the inhomogeneous terms at $\mathcal{O}\left(\alpha_{3}\right)$. We then find the well-known form of the first-order long-range

${ }^{14}$ In fact the considerations apply equally well to $\mathfrak{s u}(K)$ without modifications. 
Hamiltonian (5.8)

$$
\begin{aligned}
\mathcal{Q}_{2} & =\sum_{k=1}^{N}\left([1]_{k}-[2,1]_{k}\right)+\alpha_{3} \sum_{k=1}^{N}\left(-3[1]_{k}+4[2,1]_{k}-[3,2,1]_{k-1}\right)+\mathcal{O}\left(\lambda^{3 / 2}\right) \\
& =[1]-[2,1]+\alpha_{3}(-3[1]+4[2,1]-[3,2,1])+\mathcal{O}\left(\lambda^{3 / 2}\right) .
\end{aligned}
$$

\subsection{Beyond wrapping}

The asymptotic Bethe ansatz for long-range chains holds only as long as the range of the interactions does not exceed the length of the chain. Beyond this so-called wrapping order, not only the Bethe equations fail to provide consistent results [6], but also the construction allows for arbitrary corrections to the spectrum [37, 41]. With the above considerations one might contemplate to resolve these problems: we could simply define the undetermined wrapping interactions such that the mapping between inhomogeneous and long-range chains becomes exact. While this solves the problem of consistency, it unfortunately does not cure the arbitrariness as we shall see below.

First we replace the asymptotic Bethe equations (8.3) by the inhomogeneous ones (8.2). This is beneficial because the standard Bethe ansatz is believed to be complete: to every multiplet of eigenstates there corresponds precisely one solution of the Bethe equations. There is no doubt that this also applies for arbitrary inhomogeneities. Conversely, the asymptotic Bethe equations (8.3) are apparently not complete because the number of acceptable solutions is different for small and for large $\lambda^{15}$. Thus we have gained an exact definition of the spectrum of $\mathcal{T}(u)$ even for finite $\lambda$.

Second we choose the inhomogeneities $\mu_{k}$ in (8.23) such that (8.5) holds. However, this relation defines the inhomogeneities only up to a certain order, it is not clear how to continue (8.23) to all orders. Nevertheless, this arbitrariness is only mild, because it introduces $N$ new degrees of freedom parametrizing a spectrum of $2^{N}$ states. Furthermore, for the special choice of $\alpha_{k>3}=0[6,42]$, which is relevant to the gauge theory models discussed above, there is a natural exact choice for $\mu_{k}$ consisting only in the first term in (8.23).

Finally we have to extract the charge eigenvalues $Q_{r}$ from the transfer matrix eigenvalue $T(u)$. This is done via the integral (8.10) which we can also define to be exact in $\lambda$. There is nevertheless a residual ambiguity in this definition: the function $x(u)$ contains a branch cut and the contour of integration encircles it tightly. As long as the branch cut is infinitesimally small at $\lambda \approx 0$, there is a canonical way to define the contour. If at finite $\lambda$ the branch cut has finite extent, however, it can be deformed and the result of the contour integral will depend on the shape.

Furthermore there is the option to add corrections at $\mathcal{O}\left(\lambda^{N-r+1}\right)$ to the expression for $Q_{r}$. These corrections introduce a huge arbitrariness in that each eigenvalue can be deformed independently. This can be seen as follows: the definition (8.10) is a map from the transfer matrix eigenvalues $T(u)$ to the charges $Q_{r} . T(u)$ are normalized polynomials of degree $N$ and can thus be viewed as elements of $\mathbb{C}^{N}$, i.e. we are interested in analytic functions $\mathbb{C}^{N} \rightarrow \mathbb{C}$. It is straightforward to construct an analytic function which maps a given set of $2^{N}$ vectors (the solutions to the Bethe equations) to a given set of $N$ numbers (the spectrum). Thus with a suitable definition for the map $T(u) \mapsto Q_{r}$ we can construct any desired spectrum for $Q_{r}$.

If any desired spectrum can be obtained from an integrable spin chain model, we may wonder what is special about integrability. The point is that the map $T(u) \mapsto Q_{r}$ must enjoy additional properties: expression (8.10) ensures that only one of the two terms in the

\footnotetext{
${ }^{15}$ We thank Didina Serban for pointing out this property and for discussions.
} 
transfer matrix eigenvalue (8.8) contributes to $Q_{r}$ up to terms of order $\mathcal{O}\left(\lambda^{N-r+1}\right)$. This is the equivalent of the locality property for the corresponding spin chain charges. The logarithm of the transfer matrix $\log \mathcal{T}(u)$ has an expansion in terms of local operators $\overline{\mathcal{Q}}_{r}$ according to (8.16). Only linear combinations of $\overline{\mathcal{Q}}_{r}$ lead to local operators while nonlinear maps generate non-local terms. And indeed the map $\log T(u) \mapsto Q_{r}$ in (8.10) is linear. Unfortunately at wrapping order the notion of locality, or analogously the irrelevance of one of the two terms in (8.8), becomes meaningless. One would have to replace it by a suitable property for the $\operatorname{map} T(u) \mapsto Q_{r}$.

In conclusion, the spectrum of long-range spin chains can be adjusted arbitrarily beyond wrapping order, at least in the absence of further insights or constraints for the map $T(u) \mapsto Q_{r}$. Let us merely present a possible scenario how the arbitrariness could be cured: ideally there would be a unique way to consistently complete the map $T(u) \mapsto Q_{r}$ beyond wrapping order, which also removes the ambiguity of the integration contour. Whether or not this works remains to be seen. It would also be interesting to find out whether the bilocal deformation alias the dressing phase can somehow be incorporated into the above framework.

Let us comment on two interesting models, which could be of help in resolving the above issue. The first is the Inozemtsev spin chain which can be viewed as a long-range model in a certain limit for the coupling constant [18]. What is nice about it is that the Hamiltonian is well defined for all couplings and thus the spectrum beyond wrapping can be computed unambiguously. This model has an asymptotic Bethe ansatz, which fails already at halfwrapping. If one succeeds in completing the above transformation to an inhomogeneous Heisenberg chain, one would perhaps gain some inspiration for different models. A similar model is the Hubbard chain whose spectrum is known for all values of its coupling constant. A twisted sector was shown in [43] to be equivalent to the BDS chain (8.3), (8.9) [6] up to wrapping terms. Therefore we can again reproduce the spectrum by an inhomogeneous Heisenberg chain. In this case it is clear, however, that the two models cannot be equivalent at finite coupling: the separation of the above-mentioned sector in the Hubbard model cannot be formulated at finite coupling. In other words the Hilbert spaces are different at finite coupling; the one of the Hubbard model is considerably larger. Similarly, the Lieb-Wu equations for the Hubbard model are manifestly different from the Bethe equations for an inhomogeneous Heisenberg chain. Consequently, studying the Inozemtsev model will be more helpful for our purposes than the Hubbard model.

\section{Conclusions and outlook}

In this paper we have presented a general framework for the construction of long-range integrable spin chain models. The most important results can be summarized as follows:

- On infinite chains an integrable long-range spin chain model can be constructed to all orders in the deformation parameters.

- In the long-range Bethe ansatz the boost and bilocal deformations twist the boundary conditions which results in the rapidity map (5.10), (6.16)

$$
\left(\frac{u_{k}+\frac{\mathrm{i}}{2} t}{u_{k}-\frac{\mathrm{i}}{2} t}\right)^{L} \rightarrow\left(\frac{x\left(u_{k}+\frac{\mathrm{i}}{2} t\right)}{x\left(u_{k}-\frac{\mathrm{i}}{2} t\right)}\right)^{L}, \quad u(x)=x+\sum_{k=3}^{\infty} \frac{\alpha_{k}}{x^{k-2}},
$$

and the phase (6.35), (6.37) dressing the otherwise undeformed nearest neighbor scattering factors $S^{\mathrm{NN}}\left(u_{k}-u_{j}\right) \rightarrow S^{\mathrm{NN}}\left(u_{k}-u_{j}\right) \exp \left(2 \mathrm{i} \theta\left(u_{k}, u_{j}\right)\right)$

$$
\theta\left(u_{k}, u_{j}\right)=\sum_{s>r=2}^{\infty} \beta_{r, s}\left(q_{r}\left(u_{k}\right) q_{s}\left(u_{j}\right)-q_{s}\left(u_{k}\right) q_{r}\left(u_{j}\right)\right) .
$$


- The presented recursion relation provides a recipe to explicitly construct the long-range interactions of the integrable charges.

We shortly recapitulate the paper: starting with an arbitrary set of integrable short-range charges on infinite spin chains we have defined long-range charges by parallel transport in moduli space with respect to a connection $\Xi$. The resulting operators are manifestly integrable. Four different types of deformation operators were specified and the moduli space determined in [24] was recovered. In particular, the rapidity map parameters $\alpha_{k}$ and the dressing phase parameters $\beta_{r, s}$ were related to the boost and bilocal deformations. They change the onemagnon momenta and the two-magnon scattering phase, respectively, which is reflected in the Bethe equations. These appear to form a complete set of admissible long-range deformations of the closed Bethe equations compatible with the gauge/string structure and integrability.

A canonical basis of the charge algebra was chosen at each point of the moduli space by associating a certain change of basis to the boost deformations. This basis provides normalized charges with a minimal interaction range that grows additively in the order of each deformation parameter $\alpha_{k}, \beta_{r, s}$. In addition, the chosen change of basis renders the connection $\Xi$ on moduli space flat.

Applying our method to alternating spin chains, we have explicitly constructed a generalized Hamiltonian for the fundamental $\mathfrak{g l}\left(K_{\mathrm{e}}\right) \oplus \mathfrak{g l}\left(K_{\mathrm{o}}\right)$ spin chain. The Bethe equations for the alternating $\mathfrak{s u}(2) \oplus \mathfrak{s u}(2)$ chain were specified and gauge theory restrictions on the moduli were discussed.

Finally we have presented a correspondence between long-range and inhomogeneous spin chains. Here, it was shown how the boost-deformed long-range spin chain can be written in terms of an inhomogeneous chain. The inhomogeneity of the boost operator was thus reflected by a manifestly inhomogeneous chain.

Several questions arise in this context:

The minimization of the interaction range of the charge operators in section 5 was based on extensive experiments with the $\mathfrak{g l}(K)$ spin chain. It would be desirable to obtain a rigorous derivation of the minimizing conditions (5.22). This requires a better understanding of the range of the generated operators and its dependence on the moduli. Presumably, the conditions can be derived by methods similar to those used in appendix A.

For open long-range integrable spin chains the moduli space looks different [37]. Most notably, one finds an additional parameter $\delta_{k}$ corresponding to a phase for reflections at the boundaries of the chain. Can the recursion presented in this paper be extended to the case of open spin chains? For instance, it might seem natural to associate this phase to bilocal deformations with the boundary-counting operator $([1]-[1,2]$ in our $\mathfrak{g l}(K)$ notation) on one leg of the bilocal operator. Can we reproduce the moduli space for open chains in this fashion?

Other interesting integrable models include quantum-deformed spin chains. Again it is obvious that our construction works for these models as well. However, there certainly are further permissible deformations once only the Cartan algebra of $\mathfrak{g}$ needs to be respected. These include the Reshetikhin twist deformations [44] considered in [45].

The map between long-range and inhomogeneous spin chains was restricted to boost deformations; bilocal deformations were not included. This might have a natural origin in the inhomogeneity of the boost operator. Is it possible to map the bilocal long-range deformations to some other well-understood model of finite spin chains?

Trying to extend the presented method to sectors of $\mathcal{N}=4$ or $\mathcal{N}=6$ gauge theory beyond the $\mathfrak{s u}(2)$ sectors, two main obstacles appear: first, the underlying algebra $\mathfrak{p s u}(2,2 \mid 4)$ or $\mathfrak{o s p}(6 \mid 4)$ is no longer manifest but must receive long-range deformations in the same way as the charge operators. Even more, the Hamiltonian becomes a part of the symmetry algebra. To address these problems (cf section 3.6), one might think about joining our method with 
the ideas to deform the algebra of the $\mathfrak{s u}(1,1 \mid 2)$ sector of $\mathcal{N}=4 \mathrm{SYM}$ theory presented in [30]. Second, the length of the spin chain in other gauge theory sectors is no longer conserved. The quantum numbers of different numbers of fields coincide such that length fluctuations are admissible; the spin chain becomes dynamic [14]. A starting point to tackle this issue might be a transformation to an undynamic spin chain as presented in [46] for the $\mathfrak{s u}(2 \mid 3)$ sector of $\mathcal{N}=4 \mathrm{SYM}$. Is it possible to extend the method presented in this paper to the whole gauge/string theory algebra?

\section{Appendix A. Cancellation of longest terms}

In the construction of deformations that minimized the range of the charges, we made use of the relation (5.2), which states that, up to an overall factor, the longest-range terms of $\left[\mathcal{B}\left[\mathcal{Q}_{s}\right], \mathcal{Q}_{r}\right]$ and $\mathcal{Q}_{s+r-1}$ are the same

$$
\mathrm{i}(s-1) M\left(\left[\mathcal{B}\left[\mathcal{Q}_{s}\right], \mathcal{Q}_{r}\right]\right)=-(s+r-2) M\left(\mathcal{Q}_{s+r-1}\right) .
$$

Here, we denote by $M\left(\mathcal{L}_{k}\right)$ the part of the local operator $\mathcal{L}_{k}$ that has the longest range. In the following, we will prove the statement (A.1) for the undeformed charges $\mathcal{Q}_{r} \equiv \mathcal{Q}_{r}^{\mathrm{NN}}$ that are generated by (2.14). In this case, (A.1) reduces to

$$
(s-1) M\left(\left[\mathcal{B}\left[\mathcal{Q}_{s}\right], \mathcal{Q}_{r}\right]\right)=M\left(\left[\mathcal{B}\left[\mathcal{Q}_{2}\right], \mathcal{Q}_{s+r-2}\right]\right)
$$

In order to understand this equality, we first note that the longest-range terms of ( $s-$ 1) $\left[\mathcal{B}\left[\mathcal{Q}_{s}\right], \mathcal{Q}_{r}\right]$ equal those of $(r-1)\left[\mathcal{B}\left[\mathcal{Q}_{r}\right], \mathcal{Q}_{s}\right]$. Namely, the longest-range terms of $\left[\mathcal{B}\left[\mathcal{Q}_{s}\right], \mathcal{Q}_{r}\right]$ are given by

$$
M\left(\left[\mathcal{B}\left[\mathcal{Q}_{s}\right], \mathcal{Q}_{r}\right]\right)=(r-1) \sum_{k}\left[\mathcal{Q}_{s}(k+r-1), \mathcal{Q}_{r}(k)\right],
$$

which follows directly from the definition (3.12) and the fact that $\left[\mathcal{Q}_{s}, \mathcal{Q}_{r}\right]=0$. Using (A.3), one finds that indeed

$$
(s-1) M\left(\left[\mathcal{B}\left[\mathcal{Q}_{s}\right], \mathcal{Q}_{r}\right]\right)-(r-1) M\left(\left[\mathcal{B}\left[\mathcal{Q}_{r}\right], \mathcal{Q}_{s}\right]\right)=(s-1)(r-1) M\left(\left[\mathcal{Q}_{s}, \mathcal{Q}_{r}\right]\right)=0 .
$$

With the help of (A.4), we can now prove (A.2) inductively. For $r=2$, the equality follows directly from (A.4). Assuming that it holds for any given $r=r_{0}$, we can show that it also holds for $r=r_{0}+1$. Namely, by (A.4), one finds

$$
(s-1) M\left(\left[\mathcal{B}\left[\mathcal{Q}_{s}\right], \mathcal{Q}_{r_{0}+1}\right]\right)=r_{0} M\left(\left[\mathcal{B}\left[\mathcal{Q}_{r_{0}+1}\right], \mathcal{Q}_{s}\right]\right) .
$$

With the help of (2.14) and (A.3), this can be written as

$$
\begin{aligned}
& \frac{\mathrm{i}}{r_{0}-1} M\left(\left[\mathcal{B}\left[\mathcal{Q}_{s}\right], \mathcal{Q}_{r_{0}+1}\right]\right) \\
& =\frac{1}{(s-1)\left(r_{0}-1\right)} M\left(\left[\mathcal{B}\left[\left[\mathcal{B}\left[\mathcal{Q}_{2}\right], \mathcal{Q}_{r_{0}}\right]\right], \mathcal{Q}_{s}\right]\right) \\
& =\sum_{k}\left[\left[\mathcal{Q}_{2}\left(k+s+r_{0}-2\right), \mathcal{Q}_{r_{0}}(k+s-1)\right], \mathcal{Q}_{s}(k)\right]
\end{aligned}
$$

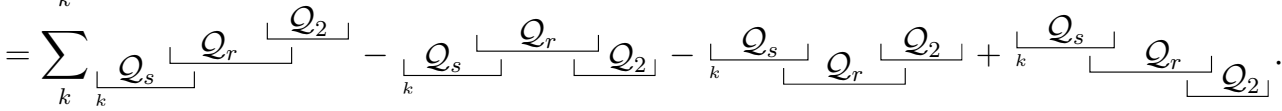


The operators in the double commutator overlap only by one site. On the other hand, by the assumption that (A.1) holds for $r=r_{0}$ and again using (A.3), one finds that

$$
\begin{aligned}
& \frac{\mathrm{i}}{(s-1)\left(r_{0}-1\right)} M\left(\left[\mathcal{B}\left[\mathcal{Q}_{2}\right], \mathcal{Q}_{s+r_{0}-1}\right]\right) \\
& =\frac{\mathrm{i}}{(s-1)\left(r_{0}-1\right)} M\left(\left[\mathcal{B}\left[\mathcal{Q}_{2}\right], M\left(\mathcal{Q}_{s+r_{0}-1}\right)\right]\right) \\
& =\frac{1}{\left(r_{0}-1\right)\left(s+r_{0}-2\right)} M\left(\left[\mathcal{B}\left[\mathcal{Q}_{2}\right], M\left(\left[\mathcal{B}\left[\mathcal{Q}_{s}\right], \mathcal{Q}_{r_{0}}\right]\right)\right]\right) \\
& =\sum_{k}\left[\mathcal{Q}_{2}\left(k+s+r_{0}-2\right),\left[\mathcal{Q}_{s}\left(k+r_{0}-1\right), \mathcal{Q}_{r_{0}}(k)\right]\right]
\end{aligned}
$$

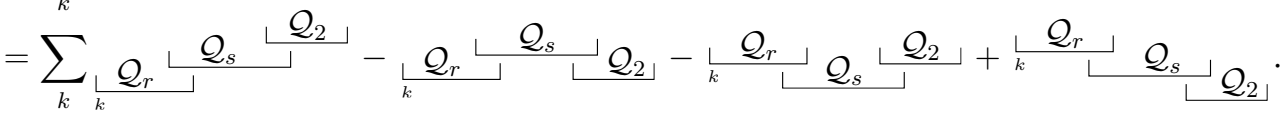

Since in the commutator under the sum in (A.6) all operators overlap only by one site, the terms can be reshuffled to yield

$$
\begin{aligned}
(s-1) M\left(\left[\mathcal{B}\left[\mathcal{Q}_{s}\right], \mathcal{Q}_{r_{0}+1}\right]\right)-M\left(\left[\mathcal{B}\left[\mathcal{Q}_{2}\right], \mathcal{Q}_{s+r_{0}-1}\right]\right) \\
=-\mathrm{i}(s-1)\left(r_{0}-1\right) \sum_{k}\left[\mathcal{Q}_{2}\left(k+s+r_{0}-2\right),\right. \\
\left.\quad\left[\mathcal{Q}_{r_{0}}(k+s-1), \mathcal{Q}_{s}(k)\right]+\left[\mathcal{Q}_{r_{0}}(k), \mathcal{Q}_{s}(k+r-1)\right]\right] \\
=-\mathrm{i}(s-1)\left(r_{0}-1\right) \sum_{k}\left[\mathcal{Q}_{2}\left(k+s+r_{0}-2\right), M\left(\left[\mathcal{Q}_{r}, \mathcal{Q}_{s}\right]\right)(k)\right] .
\end{aligned}
$$

This expression vanishes, since $M\left(\left[\mathcal{Q}_{r}, \mathcal{Q}_{s}\right]\right)=0$. Hence, (A.2) also holds for $r=r_{0}+1$, which proves (A.1) for arbitrary $r$.

\section{Appendix B. Reading off the charges}

As discussed in section 5.5, the parametrization (5.22) of the moduli space yields a flat connection $\Xi$ (4.10). Hence the charges $\mathcal{Q}_{r}$ can be directly expanded in the deformation moduli $\alpha_{k}, \beta_{r, s}$ (5.23) (up to local similarity transformations, i.e. deformations (3.8) by local operators). Taking the generating equation (4.3) as well as the redefinitions (5.22) into account, one can iteratively determine all higher order terms $\mathcal{Q}_{r}^{[\ldots]}$ of the deformed charges in terms of the undeformed charges $\mathcal{Q}_{r}^{(0)}$. By virtue of equation (2.14),

$$
\mathcal{Q}_{r+1}^{(0)}=-\frac{\mathrm{i}}{r}\left[\mathcal{B}\left[\mathcal{Q}_{2}^{(0)}\right], \mathcal{Q}_{r}^{(0)}\right],
$$

these can in turn be generated from the charge $\mathcal{Q}_{2}^{(0)}$ alone.

In the following we will explicitly carry out the expansion and express the first few terms of (5.23) in terms of the undeformed charges $\mathcal{Q}_{r}^{(0)}$. For the $\mathfrak{g l}(K)$ chain, the resulting Hamiltonian $\mathcal{Q}_{2}$ is given in table 2. Let us start with the term proportional to $\alpha_{3}$ : equation (5.4) together with the parametrization (5.9) yields

$$
\frac{\partial \mathcal{Q}}{\partial \alpha_{3}}=\mathrm{i}\left[\tilde{\mathcal{P}}_{3}, \mathcal{Q}\right]+\cdots=2 \mathrm{i}\left[\mathcal{P}_{3}, \mathcal{Q}\right]+\mathrm{i}\left[\mathfrak{G}_{3}, \mathcal{Q}\right]+\cdots,
$$

which implies

$$
\begin{aligned}
& \mathcal{Q}_{r}=\mathcal{Q}_{r}^{(0)}+\alpha_{3} \mathcal{Q}_{r}^{[3]}+\cdots \\
& \mathcal{Q}_{r}^{[3]}=2 \mathrm{i}\left[\mathcal{B}\left[\mathcal{Q}_{3}^{(0)}\right], \mathcal{Q}_{r}^{(0)}\right]+(r+1) \mathcal{Q}_{r+2}^{(0)}
\end{aligned}
$$


Similarly one finds all other terms proportional to $\alpha_{k}$

$$
\begin{aligned}
& \mathcal{Q}_{r}=\mathcal{Q}_{r}^{(0)}+\sum_{k=3}^{\infty} \alpha_{k} \mathcal{Q}_{r}^{[k]}+\cdots, \\
& \mathcal{Q}_{r}^{[k]}=(k-1) \mathrm{i}\left[\mathcal{B}\left[\mathcal{Q}_{k}^{(0)}\right], \mathcal{Q}_{r}^{(0)}\right]+(r+k-2) \mathcal{Q}_{r+k-1}^{(0)}
\end{aligned}
$$

The next thing to do is to consider terms proportional to higher powers in $\alpha_{k}$. So let us start with the term multiplied by $\alpha_{3} \alpha_{4}$ : equation (5.9) shows that

$$
\begin{aligned}
\frac{\partial \mathcal{Q}}{\partial \alpha_{3}} & =\mathrm{i}\left[\tilde{\mathcal{P}}_{3}, \mathcal{Q}\right]+(4-2) \alpha_{4} \mathrm{i}\left[\tilde{\mathcal{P}}_{3+4-1}, \mathcal{Q}\right]+\cdots \\
\frac{\partial \mathcal{Q}}{\partial \alpha_{4}} & =\mathrm{i}\left[\tilde{\mathcal{P}}_{4}, \mathcal{Q}\right]+(3-2) \alpha_{3} \mathrm{i}\left[\tilde{\mathcal{P}}_{4+3-1}, \mathcal{Q}\right]+\cdots
\end{aligned}
$$

Due to the flatness of the connection, the coefficient $\mathcal{Q}_{r}^{[4,3]}$ of $\alpha_{4}$ in $\partial \mathcal{Q} / \partial \alpha_{3}$ equals the coefficient $\mathcal{Q}_{r}^{[3,4]}$ of $\alpha_{3}$ in $\partial \mathcal{Q} / \partial \alpha_{4}$ (up to local similarity transformations), i.e.

$$
\begin{aligned}
\mathcal{Q}_{r} & =\mathcal{Q}_{r}^{(0)}+\alpha_{k} \mathcal{Q}_{r}^{[k]}+\alpha_{3} \alpha_{4} \mathcal{Q}_{r}^{[4,3]}+\cdots \\
& =\mathcal{Q}_{r}^{(0)}+\alpha_{k} \mathcal{Q}_{r}^{[k]}+\alpha_{4} \alpha_{3} \mathcal{Q}_{r}^{[3,4]}+\cdots
\end{aligned}
$$

where $\mathcal{Q}_{r}^{[34]} \simeq \mathcal{Q}_{r}^{[43]}$ and

$$
\begin{aligned}
\mathcal{Q}_{r}^{[4,3]} & =\mathrm{i}\left[\tilde{\mathcal{P}}_{3}^{[4]}, \mathcal{Q}_{r}^{(0)}\right]+\mathrm{i}\left[\tilde{\mathcal{P}}_{3}^{(0)}, \mathcal{Q}_{r}^{[4]}\right]+(4-2) \mathrm{i}\left[\tilde{\mathcal{P}}_{3+4-1}^{(0)}, \mathcal{Q}_{r}^{(0)}\right] \\
& =\mathrm{i}\left[2 \mathcal{P}_{3}^{[4]}, \mathcal{Q}_{r}^{(0)}\right]+\mathrm{i}\left[2 \mathcal{P}_{3}^{(0)}, \mathcal{Q}_{r}^{[4]}\right]+\mathrm{i}\left[\mathfrak{G}_{3}, \mathcal{Q}^{[4]}\right]_{r}+2 \mathrm{i}\left[5 \mathcal{P}_{6}^{(0)}, \mathcal{Q}_{r}^{(0)}\right]+2 \mathrm{i}\left[\mathfrak{G}_{6}, \mathcal{Q}^{(0)}\right]_{r} \\
& =2 \mathrm{i}\left[\mathcal{P}_{3}^{[4]}, \mathcal{Q}_{r}^{(0)}\right]+2 \mathrm{i}\left[\mathcal{P}_{3}^{(0)}, \mathcal{Q}_{r}^{[4]}\right]+(r+1) \mathcal{Q}_{r+2}^{[4]}+10 \mathrm{i}\left[\mathcal{P}_{6}^{(0)}, \mathcal{Q}_{r}^{(0)}\right]+2(r+4) \mathcal{Q}_{r+5}^{(0)}, \\
\mathcal{Q}_{r}^{[3,4]} & =\mathrm{i}\left[\tilde{\mathcal{P}}_{4}^{[3]}, \mathcal{Q}_{r}^{(0)}\right]+\mathrm{i}\left[\tilde{\mathcal{P}}_{4}^{(0)}, \mathcal{Q}_{r}^{[3]}\right]+(3-2) \mathrm{i}\left[\tilde{\mathcal{P}}_{4+3-1}^{(0)}, \mathcal{Q}_{r}^{(0)}\right] \\
& =\mathrm{i}\left[3 \mathcal{P}_{4}^{[3]}, \mathcal{Q}_{r}^{(0)}\right]+\mathrm{i}\left[3 \mathcal{P}_{4}^{(0)}, \mathcal{Q}_{r}^{[3]}\right]+\mathrm{i}\left[\mathfrak{G}_{4}, \mathcal{Q}^{[3]}\right]_{r}+\mathrm{i}\left[5 \mathcal{P}_{6}^{(0)}, \mathcal{Q}_{r}^{(0)}\right]+\mathrm{i}\left[\mathfrak{G}_{6}, \mathcal{Q}^{(0)}\right]_{r} \\
& =3 \mathrm{i}\left[\mathcal{P}_{4}^{[3]}, \mathcal{Q}_{r}^{(0)}\right]+3 \mathrm{i}\left[\mathcal{P}_{4}^{(0)}, \mathcal{Q}_{r}^{[3]}\right]+(r+2) \mathcal{Q}_{r+3}^{[3]}+5 \mathrm{i}\left[\mathcal{P}_{6}^{(0)}, \mathcal{Q}_{r}^{(0)}\right]+(r+4) \mathcal{Q}_{r+5}^{(0)} .
\end{aligned}
$$

In other words, the terms $\mathcal{Q}_{r}^{[4,3]}$ and $\mathcal{Q}_{r}^{[3,4]}$ determine the coefficient of $\alpha_{3} \alpha_{4}$ equally well and differ only by a local deformation (3.8). Since we have already determined $\mathcal{Q}_{r}^{[k]}$, we can plug in the result (B.4) to find

$$
\begin{gathered}
\mathcal{Q}_{r}^{[4,3]}=2 \mathrm{i}[\mathcal{B}[ \\
\left.\left.3 \mathrm{i}\left[\mathcal{P}_{4}^{(0)}, \mathcal{Q}_{3}^{(0)}\right]+5 \mathcal{Q}_{6}^{(0)}\right], \mathcal{Q}_{r}^{(0)}\right]+2 \mathrm{i}\left[\mathcal{P}_{3}^{(0)}, 3 \mathrm{i}\left[\mathcal{P}_{4}^{(0)}, \mathcal{Q}_{r}^{(0)}\right]+(r+2) \mathcal{Q}_{r+3}^{(0)}\right] \\
+(r+1)\left(3 \mathrm{i}\left[\mathcal{P}_{4}^{(0)}, \mathcal{Q}_{r+2}^{(0)}\right]+(r+4) \mathcal{Q}_{r+5}^{(0)}\right)+10 \mathrm{i}\left[\mathcal{P}_{6}^{(0)}, \mathcal{Q}_{r}^{(0)}\right]+2(r+4) \mathcal{Q}_{r+5}^{(0)} \\
\mathcal{Q}_{r}^{[3,4]}=3 \mathrm{i}\left[\mathcal{B}\left[2 \mathrm{i}\left[\mathcal{P}_{3}^{(0)}, \mathcal{Q}_{4}^{(0)}\right]+5 \mathcal{Q}_{6}^{(0)}\right], \mathcal{Q}_{r}^{(0)}\right]+3 \mathrm{i}\left[\mathcal{P}_{4}^{(0)}, 2 \mathrm{i}\left[\mathcal{P}_{3}^{(0)}, \mathcal{Q}_{r}^{(0)}\right]+(r+1) \mathcal{Q}_{r+2}^{(0)}\right] \\
+(r+2)\left(2 \mathrm{i}\left[\mathcal{P}_{3}^{(0)}, \mathcal{Q}_{r+3}^{(0)}\right]+(r+4) \mathcal{Q}_{r+5}^{(0)}\right)+5 \mathrm{i}\left[\mathcal{P}_{6}^{(0)}, \mathcal{Q}_{r}^{(0)}\right]+(r+4) \mathcal{Q}_{r+5}^{(0)} .
\end{gathered}
$$

Now the whole structure is expressed in terms of zeroth-order charges $\mathcal{Q}_{r}^{(0)}$ which can in turn be expressed in terms of $\mathcal{Q}_{2}^{(0)}$ by means of (2.14). Similarly we can continue to higher orders in the parameters $\alpha_{k}$ and will find operators that can all be expressed in terms of the simple short-range Hamiltonian.

The next step is to consider operators proportional to the free parameters $\beta_{r, s}$ : equation (5.14) yields

$$
\frac{\partial \mathcal{Q}}{\partial \beta_{r, s}}=2 \mathrm{i}\left[\mathcal{Y}_{r, s}, \mathcal{Q}\right]+\cdots=2 \mathrm{i}\left[\left[\mathcal{Q}_{r} \mid \mathcal{Q}_{s}\right], \mathcal{Q}\right]+\cdots,
$$

such that we find

$$
\mathcal{Q}_{t}=\mathcal{Q}_{t}^{(0)}+\beta_{r, s} \mathcal{Q}_{t}^{[r \mid s]}+\cdots,
$$


with

$$
\mathcal{Q}_{t}^{[r \mid s]}=2 \mathrm{i}\left[\left[\mathcal{Q}_{r}^{(0)} \mid \mathcal{Q}_{s}^{(0)}\right], \mathcal{Q}_{t}^{(0)}\right] .
$$

Higher orders in the parameters $\beta_{r, s}$ as in

$$
\mathcal{Q}_{t}=\mathcal{Q}_{t}^{(0)}+\beta_{r, s} \mathcal{Q}_{t}^{[r \mid s]}+\beta_{r, s} \beta_{p, q} \mathcal{Q}_{t}^{[r|s, p| q]}+\cdots,
$$

are then given by

$$
\mathcal{Q}_{t}^{[r|s, p| q]}=2 \mathrm{i}\left[\left[\mathcal{Q}_{r}^{[p \mid q]} \mid \mathcal{Q}_{s}^{(0)}\right], \mathcal{Q}_{t}^{(0)}\right]+2 \mathrm{i}\left[\left[\mathcal{Q}_{r}^{(0)} \mid \mathcal{Q}_{s}^{[p \mid q]}\right], \mathcal{Q}_{t}^{(0)}\right]+2 \mathrm{i}\left[\left[\mathcal{Q}_{r}^{(0)} \mid \mathcal{Q}_{s}^{(0)}\right], \mathcal{Q}_{t}^{[p \mid q]}\right],
$$

where again the flatness of the connection implies that up to local similarity transformations this term is equal to

$$
\mathcal{Q}_{t}^{[p|q, r| s]}=2 \mathrm{i}\left[\left[\mathcal{Q}_{p}^{[r \mid s]} \mid \mathcal{Q}_{q}^{(0)}\right], \mathcal{Q}_{t}^{(0)}\right]+2 \mathrm{i}\left[\left[\mathcal{Q}_{p}^{(0)} \mid \mathcal{Q}_{q}^{[r \mid s]}\right], \mathcal{Q}_{t}^{(0)}\right]+2 \mathrm{i}\left[\left[\mathcal{Q}_{p}^{(0)} \mid \mathcal{Q}_{q}^{(0)}\right], \mathcal{Q}_{t}^{[r \mid s]}\right] .
$$

Again, we could plug in the solution for the first orders (B.11) and express the whole operator in terms of the undeformed charges, and then in terms of $\mathcal{Q}_{2}^{(0)}$ by (2.14).

We are now only missing the terms $\mathcal{Q}_{t}^{[k, r \mid s]}$ proportional to mixed powers $\alpha_{k} \beta_{r, s}$ of the free parameters in

$\mathcal{Q}_{t}=\mathcal{Q}_{t}^{(0)}+\alpha_{k} \mathcal{Q}_{t}^{[k]}+\alpha_{k} \alpha_{l} \mathcal{Q}_{t}^{[k, l]}+\beta_{r, s} \mathcal{Q}_{t}^{[r \mid s]}+\beta_{r, s} \beta_{p, q} \mathcal{Q}_{t}^{[r|s, p| q]}+\alpha_{k} \beta_{r, s} \mathcal{Q}_{t}^{[k, r \mid s]}+\cdots$

Again, we can determine these either by plugging the $\beta_{r, s}$-deformation into the $\alpha_{k}$-deformation equation or vice versa. Due to flatness, both results are equal up to local deformations (3.8). The $\alpha_{k}$-equation (5.9) together with (5.15) yields

$$
\begin{aligned}
\frac{\partial \mathcal{Q}}{\partial \alpha_{k}} & =\mathrm{i}\left[\tilde{\mathcal{P}}_{k}, \mathcal{Q}\right]+\cdots \\
& =(k-1) \mathrm{i}\left[\mathcal{P}_{k}, \mathcal{Q}\right]+\mathrm{i}\left[\mathfrak{G}_{k}, \mathcal{Q}\right]-2 \beta_{r, s}\left[\left[\left[\mathfrak{G}_{k}, \mathcal{Q}\right]_{r} \mid \mathcal{Q}_{s}\right]+\left[\mathcal{Q}_{r} \mid\left[\mathfrak{G}_{k}, \mathcal{Q}\right]_{s}\right], \mathcal{Q}\right]+\cdots
\end{aligned}
$$

which results in

$$
\begin{aligned}
\mathcal{Q}_{t}^{[r \mid s, k]}=\mathrm{i}(k-1) & {\left[\mathcal{P}_{k}^{[r \mid s]}, \mathcal{Q}_{t}^{(0)}\right]+\mathrm{i}(k-1)\left[\mathcal{P}_{k}^{(0)}, \mathcal{Q}_{t}^{[r \mid s]}\right]+(t+k-2) \mathcal{Q}_{t+k-1}^{[r \mid s]} } \\
+ & 2 \mathrm{i}\left[\left[(r+k-2) \mathcal{Q}_{r+k-1}^{(0)} \mid \mathcal{Q}_{s}^{(0)}\right]+\left[\mathcal{Q}_{r}^{(0)} \mid(s+k-2) \mathcal{Q}_{s+k-1}^{(0)}\right], \mathcal{Q}_{t}^{(0)}\right]
\end{aligned}
$$

The $\beta_{r, s}$-equation (5.12) in contrast yields

$\mathcal{Q}_{t}^{[k, r \mid s]}=2 \mathrm{i}\left[\left[\mathcal{Q}_{r}^{[k]} \mid \mathcal{Q}_{s}^{(0)}\right], \mathcal{Q}_{t}^{(0)}\right]+2 \mathrm{i}\left[\left[\mathcal{Q}_{r}^{(0)} \mid \mathcal{Q}_{s}^{[k]}\right], \mathcal{Q}_{t}^{(0)}\right]+2 \mathrm{i}\left[\left[\mathcal{Q}_{r}^{(0)} \mid \mathcal{Q}_{s}^{(0)}\right], \mathcal{Q}_{t}^{[k]}\right]$.

Again, due to flatness of the connection, expressions (B.17) and (B.18) are equal up to local similarity transformations.

Note that the explicit expansions given in this appendix does not always yield terms with the desired interaction range (5.24) straightforwardly. In order to obtain terms of this minimal range, one generically has to add deformations (3.8) by some local operators. For example, when one explicitly calculates the terms (B.8) for the $\mathfrak{g l}(K)$ chain, one finds that $\mathcal{Q}_{r}^{[3,4]}$ has the minimal interaction range $(r+3)$ as given in (5.24), while the term $\mathcal{Q}_{r}^{[4,3]}$ has range $(r+4)$. Adding a deformation $\Lambda=\mathcal{L}_{4,3} \alpha_{4} \mathrm{~d} \alpha_{3}$ by some local operator $\mathcal{L}_{4,3}$ however results in $\mathcal{Q}_{r}^{[4,3]}=\mathcal{Q}_{r}^{[3,4]}$. Similarly, for all terms we calculated for the $\mathfrak{g l}(K)$ chain, (B.18) yields the correct range (5.24), while (B.17) needs to be corrected by a local deformation $\Lambda=\mathcal{L}_{r \mid s, k} \beta_{r, s} d \alpha_{k}$. For explicit results for the $\mathfrak{g l}(K)$ and $\mathfrak{g l}\left(K_{\mathrm{e}}\right) \otimes \mathfrak{g l}\left(K_{\mathrm{o}}\right)$ chains, see tables 2 and 3 . 


\section{References}

[1] Maldacena J M 1998 The large N limit of superconformal field theories and supergravity Adv. Theor. Math. Phys. 2231 (arXiv:hep-th/9711200)

Witten E 1998 Anti-de Sitter space and holography Adv. Theor. Math. Phys. 2253 (arXiv:hep-th/9802150)

[2] Minahan J A and Zarembo K 2003 The Bethe-ansatz for $\mathcal{N}=4$ super Yang-Mills J. High Energy Phys. JHEP03(2003)013 (arXiv:hep-th/0212208)

[3] Beisert N, Kristjansen C and Staudacher M 2003 The dilatation operator of $\mathcal{N}=4$ conformal super Yang-Mills theory Nucl. Phys. B 664131 (arXiv:hep-th/0303060)

[4] Bena I, Polchinski J and Roiban R 2004 Hidden symmetries of the $A d S_{5} \times S^{5}$ superstring Phys. Rev. D 69046002 (arXiv:hep-th/0305116)

[5] Beisert $N$ and Staudacher M 2003 The $\mathcal{N}=4$ SYM integrable super spin chain Nucl. Phys. B 670439 (arXiv:hep-th/0307042)

[6] Beisert N, Dippel V and Staudacher M 2004 A novel long range spin chain and planar $\mathcal{N}=4$ super Yang-Mills J. High Energy Phys. JHEP07(2004)075 (arXiv:hep-th/0405001)

[7] Minahan J A and Zarembo K 2008 The Bethe ansatz for superconformal Chern-Simons J. High Energy Phys. JHEP09(2008)040 (arXiv:0806.3951)

[8] Aharony O, Bergman O, Jafferis D L and Maldacena J $2008 \mathcal{N}=6$ superconformal Chern-Simons-matter theories, M2-branes and their gravity duals J. High Energy Phys. JHEP10(2008)091 (arXiv:0806.1218)

[9] Haldane F D M 1988 Exact Jastrow-Gutzwiller resonating valence bond ground state of the spin 1/2 antiferromagnetic Heisenberg chain with $1 / r^{2}$ exchange Phys. Rev. Lett. 60635

Shastry B S 1988 Exact solution of an $S=1 / 2$ Heisenberg antiferromagnetic chain with long ranged interactions Phys. Rev. Lett. 60639

[10] Inozemtsev V I 1990 On the connection between the one-dimensional $s=1 / 2$ Heisenberg chain and Haldane Shastry model J. Stat. Phys. 591143

Inozemtsev V I 2003 Integrable Heisenberg-van Vleck chains with variable range exchange Phys. Part. Nucl. 34166 (arXiv:hep-th/0201001)

[11] Basu-Mallick B 1996 Spin-dependent extension of Calogero-Sutherland model through anyon-like representations of permutation operators Nucl. Phys. B 482713 (arXiv:hep-th/9602107)

Basu-Mallick B 1999 Symmetries and exact solutions of some integrable Haldane-Shastry like spin chains Nucl. Phys. B 540679 (arXiv:solv-int/9809010)

[12] Beisert N 2004 The complete one-loop dilatation operator of $\mathcal{N}=4$ Super Yang-Mills theory Nucl. Phys. B 6763 (arXiv:hep-th/0307015)

[13] Bak D and Rey S-J 2008 Integrable spin chain in superconformal Chern-Simons theory J. High Energy Phys. JHEP10(2008)053 (arXiv:0807.2063)

Zwiebel B I 2009 Two-loop integrability of planar $\mathcal{N}=6$ superconformal Chern-Simons theory arXiv: 0901.0411

Minahan J A, Schulgin W and Zarembo K 2009 Two loop integrability for Chern-Simons theories with $\mathcal{N}=6$ supersymmetry J. High Energy Phys. JHEP03(2009)057 (arXiv:0901.1142)

[14] Beisert N 2004 The SU(2/3) dynamic spin chain Nucl. Phys. B 682487 (arXiv:hep-th/0310252)

[15] Zwiebel B I $2006 \mathcal{N}=4$ SYM to two loops: compact expressions for the non-compact symmetry algebra of the su(1,1/2) sector J. High Energy Phys. JHEP02(2006)055 (arXiv:hep-th/0511109)

[16] Heisenberg W 1928 Zur Theorie des Ferromagnetismus Z. Phys. 49619

[17] Bethe H 1931 Zur theorie der metalle: I. Eigenwerte und eigenfunktionen der linearen atomkette Z. Phys. 71205

[18] Serban D and Staudacher M 2004 Planar $\mathcal{N}=4$ gauge theory and the Inozemtsev long range spin chain J. High Energy Phys. JHEP06(2004)001 (arXiv:hep-th/0401057)

[19] Beisert N and Staudacher M 2005 Long-range PSU(2,2/4) Bethe ansaetze for gauge theory and strings Nucl. Phys. B 7271 (arXiv:hep-th/0504190)

[20] Gromov N and Vieira P 2009 The all loop $\mathrm{AdS}_{4} / \mathrm{CFT}_{3}$ Bethe ansatz J. High Energy Phys. JHEP01(2009)016 (arXiv:0807.0777)

[21] Ambjørn J, Janik R A and Kristjansen C 2006 Wrapping interactions and a new source of corrections to the spin-chain/string duality Nucl. Phys. B 736288 (arXiv:hep-th/0510171)

Janik R A and Lukowski T 2007 Wrapping interactions at strong coupling-the giant magnon Phys. Rev. D 76126008 (arXiv:0708.2208)

Arutyunov G and Frolov S 2007 On string S-matrix, bound states and TBA J. High Energy Phys. JHEP12(2007)024 (arXiv:0710.1568)

Minahan J A and Ohlsson Sax O 2008 Finite size effects for giant magnons on physical strings Nucl. Phys. B 80197 (arXiv:0801.2064) 
Heller M P, Janik R A and Lukowski T 2008 A new derivation of Lüscher F-term and fluctuations around the giant magnon J. High Energy Phys. JHEP06(2008)036 (arXiv:0801.4463)

Gromov N, Schafer-Nameki S and Vieira P 2008 Quantum wrapped giant magnon Phys. Rev. D 78026006 (arXiv:0801.3671)

Fiamberti F, Santambrogio A, Sieg C and Zanon D 2008 Anomalous dimension with wrapping at four loops in $\mathcal{N}=4$ SYM Nucl. Phys. B $\mathbf{8 0 5} 231$ (arXiv:0806.2095)

Bajnok Z and Janik R A 2009 Four-loop perturbative Konishi from strings and finite size effects for multiparticle states Nucl. Phys. B 807625 (arXiv:0807.0399)

Bajnok Z, Janik R A and Lukowski T 2009 Four loop twist two, BFKL, wrapping and strings Nucl. Phys. B 816376 (arXiv:0811.4448)

[22] Gromov N, Kazakov V and Vieira P 2009 Integrability for the full spectrum of planar AdS/CFT arXiv:0901.3753

[23] Beccaria M, Forini V, Lukowski T and Zieme S 2009 Twist-three at five loops, Bethe ansatz and wrapping $J$. High Energy Phys. JHEP03(2009)129 (arXiv:0901.4864)

[24] Beisert N and Klose T 2006 Long-range $G L(n)$ integrable spin chains and plane-wave matrix theory J. Stat. Mech. 06 P07006 (arXiv:hep-th/0510124)

[25] Beisert N and Erkal D 2008 Yangian symmetry of long-range gl(N) integrable spin chains J. Stat. Mech. 0803 P03001 (arXiv:0711.4813)

[26] Bargheer T, Beisert N and Loebbert F 2008 Boosting nearest-neighbour to long-range integrable spin chains J. Stat. Mech. 0811 L11001 (arXiv:0807.5081)

[27] Drinfel'd V G 1985 Hopf algebras and the quantum Yang-Baxter equation Sov. Math. Dokl. 32254

[28] Drinfel'd V G 1988 Quantum groups J. Math. Sci. 41898

[29] Sogo K and Wadati M 1983 Boost operator and its application to quantum Gelfand-Levitan equation for Heisenberg-Ising chain with spin one-half Prog. Theor. Phys. 69431

[30] Zwiebel B I 2008 Iterative structure of the $\mathcal{N}=4$ SYM spin chain J. High Energy Phys. JHEP07(2008)114 (arXiv:0806.1786)

[31] Beisert N, McLoughlin T and Roiban R 2007 The four-loop dressing phase of $\mathcal{N}=6$ SYM Phys. Rev. D 76046002 (arXiv:0705.0321)

[32] Sutherland B 1978 A brief history of the quantum soliton with new results on the quantization of the Toda lattice Rocky Mt. J. Math. 8413

[33] Staudacher M 2005 The factorized S-matrix of CFT/AdS J. High Energy Phys. JHEP05(2005)054 (arXiv:hep-th/0412188)

[34] Arutyunov G, Frolov S and Staudacher M 2004 Bethe ansatz for quantum strings J. High Energy Phys. JHEP10(2004)016 (arXiv:hep-th/0406256)

[35] Beisert N 2005 Spin chain for quantum strings Fortsch. Phys. 53852 (arXiv:hep-th/0409054)

[36] Reshetikhin N Yu 1983 A method of functional equations in the theory of exactly solvable quantum system Lett. Math. Phys. 7205

Reshetikhin N Yu 1985 Integrable models of quantum one-dimensional magnets with $\mathrm{O}(\mathrm{N})$ and $\operatorname{Sp}(2 \mathrm{~K})$ symmetry Theor. Math. Phys. 63555

Ogievetsky E and Wiegmann P 1986 Factorized S matrix and the Bethe ansatz for simple Lie groups Phys. Lett. B 168360

[37] Beisert N and Loebbert F 2008 Open perturbatively long-range integrable gl(N) spin chains arXiv:0805.3260

[38] Teschner J 2008 On the spectrum of the Sinh-Gordon model in finite volume Nucl. Phys. B 799403 (arXiv:hep-th/0702214)

[39] Faddeev L D and Reshetikhin N Yu 1986 Integrability of the principal chiral field model in 1+1 dimension Ann. Phys. 167227

Destri C and de Vega H J 1989 Light-cone lattices and the exact solution of chiral fermion and sigma models J. Phys. A: Math. Gen. 221329

de Vega H J and Woynarovich F 1992 New integrable quantum chains combining different kinds of spins J. Phys. A: Math. Gen. 254499

Abad J and Ríos M 1997 Integrable su(3) spin chain combining different representations J. Phys. A: Math. Gen. 305887 (arXiv:cond-mat/9706136)

Martins M J 1999 Integrable mixed vertex models from braid-monoid algebra arXiv:solv-int/9903006

[40] Kotikov A V, Lipatov L N, Rej A, Staudacher M and Velizhanin V N 2007 Dressing and wrapping J. Stat. Mech. 0710 P10003 (arXiv:0704.3586)

[41] Beisert N 2004 The dilatation operator of $\mathcal{N}=4$ super Yang-Mills theory and integrability Phys. Rept. 4051 (arXiv:hep-th/0407277)

[42] Beisert N 2008 The su(2/2) dynamic S-matrix Adv. Theor. Math. Phys. 12945 (arXiv:hep-th/0511082)

[43] Rej A, Serban D and Staudacher M 2006 Planar $\mathcal{N}=4$ gauge theory and the Hubbard model J. High Energy 
Phys. JHEP03(2006)018 (arXiv:hep-th/0512077)

[44] Reshetikhin N 1990 Multiparameter quantum groups and twisted quasitriangular Hopf algebras Lett. Math. Phys. 20331

[45] Roiban R 2004 On spin chains and field theories J. High Energy Phys. JHEP09(2004)023 (arXiv:hep-th/0312218)

Berenstein D and Cherkis S A 2004 Deformations of $\mathcal{N}=4$ SYM and integrable spin chain models Nucl. Phys. B 70249 (arXiv:hep-th/0405215)

Frolov S A, Roiban R and Tseytlin A A 2005 Gauge-string duality for superconformal deformations of $\mathcal{N}=4$ super Yang-Mills theory J. High Energy Phys. JHEP07(2005)045 (arXiv:hep-th/0503192)

Beisert N and Roiban R 2005 Beauty and the twist: the Bethe ansatz for twisted $\mathcal{N}=4$ SYM J. High Energy Phys. JHEP08(2005)039 (arXiv:hep-th/0505187)

[46] Beisert N 2008 The su(2/3) undynamic spin chain arXiv:0807.0099 